\title{
Posets of finite functions
}

\author{
Konrad Pióro \\ Institute of Mathematics \\ University of Warsaw \\ Warsaw, Poland \\ kpioro@mimuw.edu.pl
}

Submitted: May 22, 2015; Accepted: Dec 22, 2015; Published: Jan 11, 2016

Mathematics Subject Classifications: 06A07, 06B23

\begin{abstract}
The symmetric group $S(n)$ is partially ordered by Bruhat order. This order is extended by L. Renner to the set of partial injective functions of $\{1,2, \ldots, n\}$ (see, Linear Algebraic Monoids, Springer, 2005). This poset is investigated by M. Fortin in his paper The MacNeille Completion of the Poset of Partial Injective Functions [Electron. J. Combin., 15, R62, 2008]. In this paper we show that Renner order can be also defined for sets of all functions, partial functions, injective and partial injective functions from $\{1,2, \ldots, n\}$ to $\{1,2, \ldots, m\}$. Next, we generalize Fortin's results on these posets, and also, using simple facts and methods of linear algebra, we give simpler and shorter proofs of some fundamental Fortin's results. We first show that these four posets can be order embedded in the set of $n \times m$-matrices with non-negative integer entries and with the natural componentwise order. Second, matrix representations of the Dedekind-MacNeille completions of our posets are given. Third, we find join- and meet-irreducible elements for every finite sublattice of the lattice of all $n \times m$-matrices with integer entries. In particular, we obtain join- and meet-irreducible elements of these Dedekind-MacNeille completions. Hence and by general results concerning Dedekind-MacNeille completions, join- and meet-irreducible elements of our four posets of functions are also found. Moreover, subposets induced by these irreducible elements are precisely described.

Keywords: finite functions, partial functions, partial injective functions, matrix representation of function, Bruhat order, Renner order, posets, posets of functions, posets of matrices, join-irreducible element, meet-irreducible element, DedekindMacNeille completion
\end{abstract}

\section{Introduction}

The symmetric group $S(n)$ of all bijections of the finite set $\{1,2, \ldots, n\}$ is partially ordered by Bruhat order (see [1]). The Dedekind-MacNeille completion of $S(n)$ (i.e., the 
smallest lattice that contains $S(n)$ ) is characterized (up to isomorphism) in [10] as some finite lattice of square matrices of size $n$ with non-negative integer entries and with the natural componentwise order. Hence it is obtained in [10] that this completion has the same number of elements as the set of all alternating sign matrices (see [2]). The Bruhat order is extended by Renner in [12] for the set $P(n)$ of all partial injective functions of $\{1,2, \ldots, n\}$ (the partiality of a function means that its domain is a subset of $\{1,2, \ldots, n\}$ ). The poset $P(n)$ is investigated by M. Fortin in [7] (see also [6]). He first proves that $P(n)$ is isomorphic to a poset of some special square matrices of size $n$ with non-negative integer entries, ordered componentwise. Next, this poset is extended in a natural way to a lattice of square matrices. Hence a square matrix representation of Dedekind-MacNeille completion $L(P(n))$ of $P(n)$ is obtained. This representation shows that $L(P(n))$ is distributive and has the same number of elements as the set of all alternating matrices, where these matrices generalize the alternating sign matrices. Moreover, having this matrix characterization M. Fortin finds join- and meet-irreducible elements of $L(P(n))$. Thus by general results concerning Dedekind-MacNeille completions he also obtains descriptions of joinand meet-irreducible elements of $P(n)$.

In the present paper we show that Renner order can be also defined for sets of all functions, partial functions, injective and partial injective functions from $\{1,2, \ldots, n\}$ to $\{1,2, \ldots, m\}$, where $n, m$ are arbitrary positive integers. Next, we generalize Fortin's results on these four posets, and also, using simple facts and methods of linear algebra (e.g., classical operations on rows and columns of matrices), we give simpler and shorter proofs of some fundamental results of [7]. We first show that each function can be represented by an $n \times m$-matrix with non-negative integer entries. Having this matrix representation we show that our posets can be order embedded in the poset of all $n \times m$-matrices with non-negative integer entries and with the natural componentwise order. Secondly, matrix representations of Dedekind-MacNeille completions of our posets are given. In particular, we obtain another matrix representation of the Dedekind-MacNeille completion of the symmetric group $S(n)$ than that given in [10]. Thirdly, we find join- and meet-irreducible elements for every finite sublattice of the lattice of all $n \times m$-matrices with integer entries. In particular, join- and meet-irreducible elements of these Dedekind-MacNeille completions are found. Hence we obtain also join- and meet-irreducible elements of our four function posets. Moreover, structures of subposets induced by these irreducible elements are precisely described.

\subsection{Basic definitions and facts}

We recall a few definitions and facts concerning posets (partially ordered sets) and lattices needed in this paper (for details see $[4,5,9,13])$. Take posets $\left(P, \leqslant_{P}\right),\left(Q, \leqslant_{Q}\right)$ and let $f: P \longrightarrow Q$ be a function. Then $f$ is an (order) embedding if, for each $p_{1}, p_{2} \in P$, $p_{1} \leqslant P p_{2}$ iff $f\left(p_{1}\right) \leqslant_{Q} f\left(p_{2}\right)$. In particular, each embedding is injective. If additionally $f$ is surjective, then $f$ is an isomorphism of $P$ on $Q$ (or an automorphism of $P$ if $Q=P$ ).

Let $R \subseteq P$ be a subset of a poset $\left(P, \leqslant_{P}\right)$. Then we can restrict $\leqslant_{P}$ to $R$ and we obtain a poset $\left(R, \leqslant_{P \mid R}\right)$ which is called an induced subposet, or simply a subposet, of $\left(P, \leqslant_{P}\right)$ (see [13], p. 243). To simplify notation we will write $\left(R, \leqslant_{P}\right)$ instead of $\left(R, \leqslant_{P \uparrow R}\right)$. Of 
course, $\left(Q, \leqslant_{Q}\right)$ is a subposet of $\left(P, \leqslant_{P}\right)$ iff $Q \subseteq P$ and the identity embedding $Q \hookrightarrow P$ is an order embedding.

Take a poset $\left(P, \leqslant_{P}\right)$ and its subset $X \subseteq P$. Then $\bigvee X$ and $\bigwedge X$ denote the supremum and the infimum of $X$ (if exist) in $P$, respectively. If $X=\left\{x_{1}, x_{2}\right\}$, then we write $x_{1} \vee x_{2}$ and $x_{1} \wedge x_{2}$ instead of $\bigvee\left\{x_{1}, x_{2}\right\}$ and $\bigwedge\left\{x_{1}, x_{2}\right\}$, respectively. Recall that a poset $\left(L, \leqslant_{L}\right)$ is a complete lattice (a lattice) if each set (each two-element set) has a supremum and an infimum. In particular, a complete lattice has the bottom element $\mathbf{0}_{L}=\bigvee \emptyset$ and the top element $\mathbf{1}_{L}=\bigwedge \emptyset$. Each finite lattice is complete.

(Completely) join- and meet-irreducibility are classical notions of lattice theory (see [4] and [9]), which are also useful in the theory of posets (see [8, 10, 11]). An element $i \in P$ is (completely) join-irreducible if, for each set $Y \subseteq P$ such that $\bigvee Y$ exists, $i=\bigvee Y$ implies $i \in Y$. In particular, the least element $\mathbf{0}_{P}$ of $P$ (if exists) is not join-irreducible, because $\bigvee \emptyset=\mathbf{0}_{P}$. Otherwise, if $P$ has no least element, then each minimal element of $P$ is join-irreducible. The set of all (completely) join-irreducible elements of poset $\left(P, \leqslant_{P}\right)$ will be denoted by $\operatorname{Jir}(P)$. An element $i \in P$ is (completely) meet-irreducible if, for each set $Y \subseteq P$ such that $\bigwedge Y$ exists, $i=\bigwedge Y$ implies $i \in Y$. In particular, the greatest element $\mathbf{1}_{P}$ of $P$ (if exists) is not meet-irreducible, because $\bigwedge \emptyset=\mathbf{1}_{P}$. Otherwise, if $P$ has no greatest element, then each maximal element of $P$ is meet-irreducible. The set of all (completely) meet-irreducible elements of poset $\left(P, \leqslant_{P}\right)$ will be denoted by $\operatorname{Mir}(P)$.

If $\left(P, \leqslant_{P}\right)$ is a complete lattice, then $\bigvee Y$ and $\wedge Y$ exist for all $Y \subseteq P$ and we obtain classical lattice definitions of (completely) join- and meet-irreducible elements (see [4] and [9]). In lattice theory we have also weaker notions of join- and meet-irreducibility (see also [4] and [9]), which are obtained by taking two-element sets in the above two definitions. These notions are too weak in the case of posets, and they are not used in this paper. For example, take a poset $P=\left\{x_{1}, x_{2}, x_{3}, y_{1}, y_{2}, y_{3}, y_{4}\right\}$ such that $x_{1}, x_{2} \leqslant_{P} y_{1}, x_{1}, x_{3} \leqslant_{P} y_{4}$, $x_{2}, x_{3} \leqslant_{P} y_{3}$ and $x_{1}, x_{2}, x_{3} \leqslant_{P} y_{2}$. Then $\bigvee\left\{x_{1}, x_{2}, x_{3}\right\}=y_{2}$, so $y_{2}$ is not completely joinirreducible. But it is easy to verify that $y_{2}$ is join-irreducible. On the other hand, for a finite lattice $\left(L, \leqslant_{L}\right)$ we have that its element $l \in L$ is completely join-(meet-)irreducible if $l \neq \mathbf{0}_{L}\left(l \neq \mathbf{1}_{L}\right)$ and $l$ is join-(meet-)irreducible, i.e., for each $k_{1}, k_{2} \in L, l=k_{1} \vee k_{2}$ $\left(l=k_{1} \wedge k_{2}\right)$ implies $l=k_{1}$ or $l=k_{2}$. Since almost all lattices in this paper are finite and for posets we use only one kind of join- and meet-irreducibility, we will omit the adverb "completely" in phrases completely join-irreducible and completely meet-irreducible.

Let $\leqslant_{P}^{d}$ denotes the inverse relation to $\leqslant_{P}$, i.e., $p \leqslant_{P}^{d} q$ iff $q \leqslant_{P} p$ for all $p, q \in P$. Then $\leqslant_{P}^{d}$ is also a partial order on $P$. Recall that $\left(P, \leqslant_{P}^{d}\right)$ is called the dual poset and is sometimes denoted by $P^{d}$ (see [4]). Obviously, an element is join-irreducible (respectively, meet-irreducible) in $\left(P, \leqslant_{P}\right)$ iff it is meet-irreducible (respectively, join-irreducible) in $\left(P, \leqslant_{P}^{d}\right)$. A poset $\left(P, \leqslant_{P}\right)$ is called self-dual if $\left(P, \leqslant_{P}\right)$ is isomorphic to its dual $\left(P, \leqslant_{P}^{d}\right)$. Isomorphisms between $P$ and its dual $P^{d}$ are sometimes called anti-automorphisms of $P$. Thus $P$ is self-dual iff there is an anti-automorphism of $P$. If $P$ is self-dual, then $\left(\operatorname{Jir}(P), \leqslant_{P}\right) \simeq\left(\operatorname{Jir}\left(P^{d}\right), \leqslant_{P}^{d}\right)=\left(\operatorname{Mir}(P), \leqslant_{P}^{d}\right)$ and $\left(\operatorname{Mir}(P), \leqslant_{P}\right) \simeq\left(\operatorname{Mir}\left(P^{d}\right), \leqslant_{P}^{d}\right)=$ $\left(\operatorname{Jir}(P), \leqslant_{P}^{d}\right)$. It is the well-known fact (see [4]) that if $\left(L, \leqslant_{L}\right)$ is a lattice, then its dual $\left(L, \leqslant_{L}^{d}\right)$ is also a lattice, where the join of $\left(L, \leqslant_{L}\right)$ is the meet of $\left(L, \leqslant_{L}^{d}\right)$ and the meet of $\left(L, \leqslant_{L}\right)$ is the join of $\left(L, \leqslant_{L}^{d}\right)$. 
A non-empty subset $R \subseteq P$ is called an order ideal of $\left(P, \leqslant_{P}\right)$ if, for each $p, q \in P$, $p \leqslant_{P} q \in R$ implies $p \in R$ (see [4] and [9]). A non-empty subset $S \subseteq P$ is called an order filter of $\left(P, \leqslant_{P}\right)$ if, for each $p, r \in P, S \ni r \leqslant_{P} p$ implies $p \in S$ (see [4] and [9]). Families of all order ideals and order filters of $\left(P, \leqslant_{P}\right)$, together with the empty set $\emptyset$, will be denoted by $O I(P)$ and $O F(P)$, respectively. Then $(O I(P), \subseteq)$ and $(O F(P), \subseteq)$ with set inclusion form distributive complete lattices (see also [4] and [9]), where, for each family $\mathcal{F}$ of order ideals or order filters, its infimum $\bigwedge \mathcal{F}$ is the intersection $\bigcap \mathcal{F}$ of this family and its supremum $\bigvee \mathcal{F}$ is the union $\bigcup \mathcal{F}$ of this family. It is a classical result (see [9] for details) that each finite distributive lattice $\left(L, \leqslant_{L}\right)$ is isomorphic to $(O I(\operatorname{Jir}(L)), \subseteq)$, so is also anti-isomorphic to $(O F(\operatorname{Mir}(L)), \subseteq)$ by duality.

Assume that $\left(P, \leqslant_{P}\right)$ is a finite poset and recall (see [13], Chapter 3$)$ that there is a natural bijection between order ideals and anti-chains (i.e., subsets of pairwise incomparable elements) of $\left(P, \leqslant_{P}\right)$. More precisely, take an order ideal $R \subseteq P$ and let $X_{R}$ be the set of all maximal elements of the subposet $\left(R, \leqslant_{P}\right)$. Then $R=\bigcup_{x \in X_{R}}\left\{p \in P: p \leqslant_{P} x\right\}$, because $R$ is finite. Hence $R$ is uniquely determined by $X_{R}$. Moreover, $X_{R}$ is an anti-chain in $\left(P, \leqslant_{P}\right)$. On the other hand, for each anti-chain $X$ in $\left(P, \leqslant_{P}\right), R_{X}=\bigcup_{x \in X}\left\{p \in P: p \leqslant_{P} x\right\}$ is an order ideal of $\left(P, \leqslant_{P}\right)$ such that $X_{R_{X}}=X$. Thus we have a bijection between order ideals of finite poset and anti-chains of this poset. By duality we obtain also analogous facts for order filters of $\left(P, \leqslant_{P}\right)$. Each order filter $S \subseteq P$ is uniquely determined by the set $Y_{S}$ of all minimal elements of $\left(S, \leqslant_{P}\right)$ (note that $S=\bigcup_{y \in Y_{S}}\left\{q \in P: y \leqslant_{P} q\right\}$ ), and $Y_{S}$ is an anti-chain. Moreover, for each anti-chain $Y, S_{Y}=\bigcup_{y \in Y}\{q \in P: y \leqslant P q\}$ is an order filter such that $Y_{S_{Y}}=Y$. In particular, there is a bijection between order filters of finite poset and anti-chains of this poset. By these facts we obtain that the number of elements of a finite distributive lattice $\left(L, \leqslant_{L}\right)$ is equal to the number of all anti-chains of $\left(\operatorname{Jir}(L), \leqslant_{L}\right)$, and also is equal to the number of all anti-chains of $\left(\operatorname{Mir}(L), \leqslant_{L}\right)$.

\subsection{Posets of functions}

$\mathbb{Z}, \mathbb{N}$ and $\mathbb{N}^{*}=\mathbb{N} \backslash\{0\}$ are sets of all integers, non-negative integers and positive integers, respectively. The natural linear order on $\mathbb{Z}$, thus also on $\mathbb{N}$, will be denoted by $\leqslant$. Next, product orders induced by $\leqslant$ on direct powers $\mathbb{Z}^{n}$ and $\mathbb{N}^{n}$, where $n \in \mathbb{N}^{*}$, will be denoted by $\leqslant_{\text {prod }}$ (it is not precise notation, but it should not give reason to misunderstanding).

Let $A$ and $B$ be sets. A function $f$ from any subset $X \subseteq A$ to $B$ is called a partial function from $A$ to $B$ and denoted by $f: A \rightarrow B$; its domain is denoted by $\operatorname{dom}(f)$, i.e., $\operatorname{dom}(f)=X$.

Definition 1. For all positive integers $n, m \in \mathbb{N}^{*}$,

(a) Let $F(n, m)$ be a family of all functions from $\{1,2, \ldots, n\}$ to $\{0,1, \ldots, m\}$.

(b) Let $P(n, m)$ be a family of all injective partial functions from $\{1,2, \ldots, n\}$ to $\{1,2, \ldots, m\}$.

(c) Let $T(n, m)$ be a family of all functions from $\{1,2, \ldots, n\}$ to $\{1,2, \ldots, m\}$. 
(d) Let $I(n, m)=P(n, m) \cap T(n, m)$, i.e., $I(n, m)$ is the family of all injective functions from $\{1,2, \ldots, n\}$ to $\{1,2, \ldots, m\}$.

If $n=m$, then we will write $F(n), P(n)$ and $T(n)$ instead of $F(n, n), P(n, n)$ and $T(n, n)$, respectively. Moreover, $I(n, n)$ will be denoted by $S(n)$.

Of course, $I(n, m) \subseteq T(n, m) \subseteq F(n, m), I(n, m) \subseteq P(n, m)$ and $|F(n, m)|=(m+1)^{n}$, $|T(n, m)|=m^{n},|I(n, m)|=m \cdot(m-1) \cdot \ldots \cdot(m-n+1)$. If $m<n$, then $I(n, m)=\emptyset$ and each $f \in P(n, m)$ is a partial function whose domain has at most $m$ elements. If $n=m$, then $I(n, n)$ is the family of all bijections of $\{1,2,3, \ldots, n\}$, and therefore it is denoted by $S(n)$. Of course, $|S(n)|=n$ !. Next, each function $f \in P(n, m)$ can be considered as a function from $\{1,2, \ldots, n\}$ to $\{0,1, \ldots, m\}$ by setting $f(i)=0$ for all $i \in\{1,2,3, \ldots, n\} \backslash \operatorname{dom}(f)$ (see [7]). Thus $P(n, m) \subseteq F(n, m)$ and $|P(n, m)|=$ $1+\sum_{k=1}^{n}\left(\begin{array}{l}n \\ k\end{array}\right) \cdot m \cdot(m-1) \cdot(m-2) \cdot \ldots \cdot(m-k+1)$ (here 1 corresponds to the empty partial injective function).

Similarly as in the case of partial injective functions we can consider arbitrary partial functions from $\{1,2,3, \ldots, n\}$ to $\{1,2,3, \ldots, m\}$ as elements of $F(n, m)$, and vice versa, each $f \in F(n, m)$ is a partial function such that $\operatorname{dom}(f)=\{1,2,3, \ldots, n\} \backslash\{i: f(i)=0\}$. Hence the set $F(n, m)$ can be considered as the family of all partial functions from $\{1,2,3, \ldots, n\}$ to $\{1,2,3, \ldots, m\}$. But it is no important idea, because the function $\Theta:(f(1), f(2), \ldots, f(n)) \longmapsto(f(1)+1, f(2)+1, \ldots, f(n)+1)$ is a natural bijection between $F(n, m)$ and $T(n, m+1)$. Moreover, we will show below that these two sets form isomorphic posets.

The set $S(n)$ is partially ordered by Bruhat order (see [1]). First, the relation $\rightarrow_{B}$ is defined on $S(n)$ as follows: $f \rightarrow_{B} g$ if there are $1 \leqslant i<j \leqslant n$ such that $f(i)<f(j)$ and $g$ is obtained from $f$ by transposing $f(i)$ and $f(j)$, i.e., $g(i)=f(j), g(j)=f(i)$ and $g(k)=f(k)$ for all $k \neq i, j$. If $f \rightarrow_{B} g$, then $g$ has one more inversion than $f$. Recall that a pair $(i, j)$ is an inversion of $h \in F(n, m)$ if $i<j$ and $h(i)>h(j)$. Hence $\rightarrow_{B}$ and its transitive closure are antisymmetric. Thus the transitive closure of $\rightarrow_{B}$, together with the identity relation, forms a partial order $\leqslant_{B}$ called Bruhat order.

The relation $\rightarrow_{B}$ and Bruhat order $\leqslant_{B}$ can be defined on the set $F(n, m)$, thus on $P(n, m), I(n, m)$ and $T(n, m)$ too, without changes. But Pennell, Puchta and Renner have defined a relation $\rightarrow$ on $P(n)$ which generalizes $\rightarrow_{B}$. Consequently, they have obtained a partial order on $P(n)$ which generalizes Bruhat order (see [12], Sections 8.7 and 8.8, see also [7]). This relation $\rightarrow$ and its partial order can be defined on $F(n, m)$ without changes. Recall that $f \rightarrow g$ if one of the following two conditions hold:

(o.1) there are $1 \leqslant i, j \leqslant n$ such that $i<j, g(j)=f(i)<f(j)=g(i)$ and $f(k)=g(k)$ for all $k \neq i, j$;

(o.2) there is $1 \leqslant i \leqslant n$ such that $f(i)<g(i)$ and $f(j)=g(j)$ for all $j \neq i$.

For $f \in F(n, m), L(f)$ is the number of all inversions of $f$ plus the sum $\sum_{k=1}^{n} f(k)$ (see [7]). If $f \rightarrow g$, then $L(f)<L(g)$. Hence $\rightarrow$ and its transitive closure are antisymmetric. Thus the transitive closure of $\rightarrow$, together with the identity relation, forms a partial order 
on $F(n, m)$. This order is denoted by $\leqslant_{F}$, or more formally by $\leqslant_{F(n, m)}$. We have also three subposets of $\left(F(n, m), \leqslant_{F}\right)$ induced by sets $I(n, m), P(n, m)$ and $T(n, m)$. It is easy to see that the natural bijection $\Theta$ is an isomorphism between $\left(F(n, m), \leqslant_{F}\right)$ and $\left(T(n, m+1), \leqslant_{F}\right)$. Next, for $n=m$ we have the subposet $\left(S(n), \leqslant_{F}\right)$ of $\left(F(n, m), \leqslant_{F}\right)$. But it is easy to see that (o.2) cannot holds for bijections. Thus the restriction of $\leqslant_{F}$ to $S(n)$ is equal to Bruhat order $\leqslant_{B}$, so $\left(S(n), \leqslant_{F}\right)=\left(S(n), \leqslant_{B}\right)$. Of course, $\left(S(n), \leqslant_{B}\right)$ is also a subposet of $\left(P(n), \leqslant_{F}\right)$ and $\left(T(n), \leqslant_{F}\right)$.

The set $F(n, m)$ can be also considered as the direct power $\{0,1, \ldots, m\}^{n}$. Since $\{0,1, \ldots, m\}$ is linearly ordered by the standard order $\leqslant$ on $\mathbb{N}$, we have the product order $\leqslant_{\text {prod }}$ on $F(n, m)$, i.e, $f \leqslant_{\text {prod }} g$ iff $f(i) \leqslant g(i)$ for all $1 \leqslant i \leqslant n$. It is easy to see that $\leqslant_{F}$ is transitive closure of the union $\leqslant_{\text {prod }} \cup \rightarrow_{B}$.

It is well-known that $\left(P(n, m), \leqslant_{P}\right)$ and $\left(S(n), \leqslant_{B}\right)$ are not lattices in general (see [1] and [7]). The similar fact holds for $\left(F(n, m), \leqslant_{F}\right)$, so also for $\left(T(n, m), \leqslant_{F}\right)$. Take posets $P(2), F(2)$ and $S(3)$ (the poset $S(2)$ is a two-element chain, so it is a lattice). Then $\{(1,0),(0,2)\}$, which is contained in $P(2)$ and $F(2)$ simultaneously, has no supremum in both these posets. Next, $(2,1,3)$ and $(1,3,2)$ has no supremum in $S(3)$.
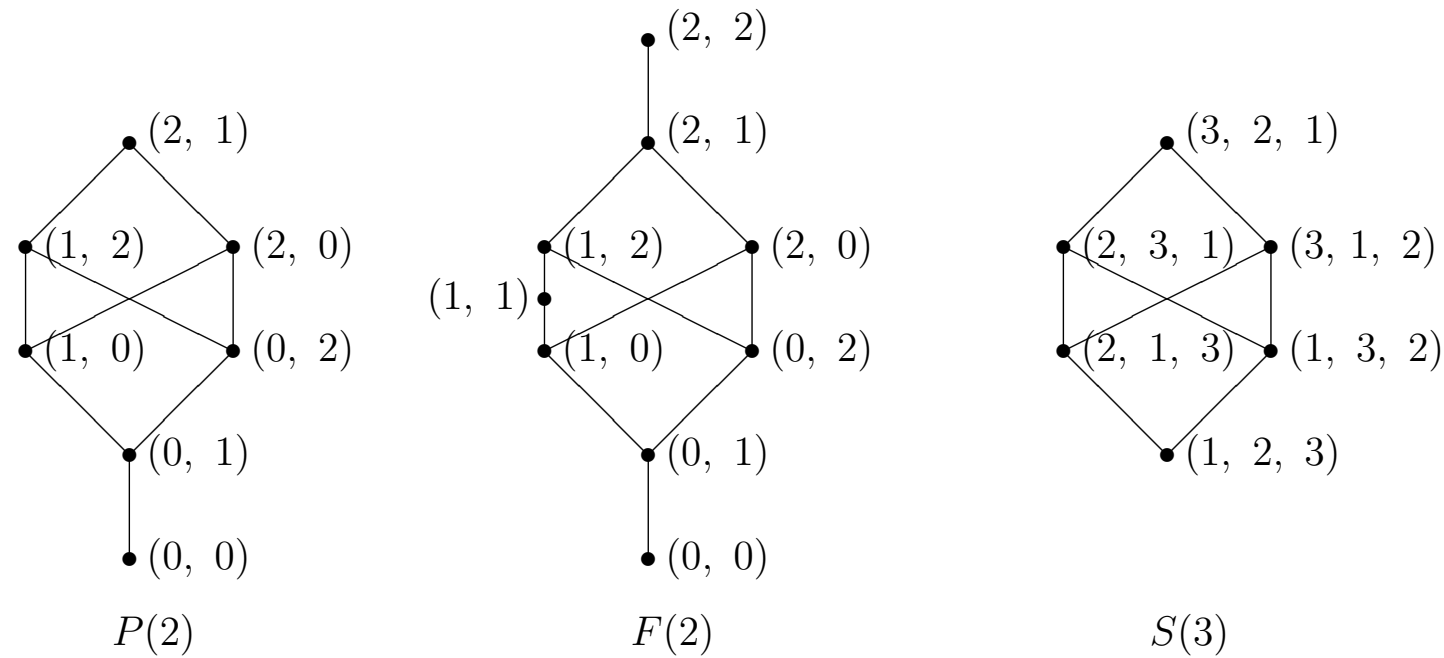

For all $m \in \mathbb{N}^{*}, F(1, m)=P(1, m), T(1, m)=I(1, m)$ and these sets are linearly ordered by $\leqslant_{F}$. Next, for each $n \in \mathbb{N}^{*}$ we have

$$
P(n, 1)=\{(0,0, \ldots, 0,0),(1,0, \ldots, 0,0),(0,1, \ldots, 0,0), \ldots,(0,0, \ldots, 0,1)\}
$$

note that $(0,0, \ldots, 0,0)$ denotes the empty partial injective function. Thus $\left(P(n, 1), \leqslant_{F}\right)$ is an $n+1$-element chain, because $(0,0, \ldots, 0,0) \leqslant$ prod $(0,0, \ldots, 0,1) \rightarrow(0,0, \ldots, 1,0) \rightarrow$ $\ldots \rightarrow(0,1, \ldots, 0,0) \rightarrow(1,0, \ldots, 0,0)$. We will see in Subsection 2.3 that $\left(F(n, 1), \leqslant_{F}\right)$, for all $n \in \mathbb{N}^{*}$, is a lattice (but linearly ordered only for $n=1,2$ ). We will also show that $\left(F(n, m), \leqslant_{F}\right)$ and $\left(P(n, m), \leqslant_{F}\right)$ are not lattices for $n, m \geqslant 2$. Moreover, $\left(I(n, m), \leqslant_{F}\right)$ is a lattice only for $m=n=2$.

Posets $\left(F(n, m), \leqslant_{F}\right),\left(T(n, m), \leqslant_{P}\right),\left(P(n, m), \leqslant_{P}\right)$ and $\left(I(n, m), \leqslant_{I}\right)$, for $n, m \in \mathbb{N}^{*}$, 
have the bottom and the top elements. Functions $\mathbf{0}_{n}=(\underbrace{0,0, \ldots, 0}_{n})$ and $\mathbf{1}_{n}=(\underbrace{1,1, \ldots, 1}_{n})$ are the bottom elements of $\left(F(n, m), \leqslant_{F}\right)$ and $\left(T(n, m), \leqslant_{P}\right)$, respectively. Next, $\mathbf{m}_{n}=$ $(\underbrace{m, m, \ldots, m}_{n})$ is the top element of $\left(F(n, m), \leqslant_{F}\right)$ and $\left(T(n, m), \leqslant_{P}\right)$.

Since $\mathbf{0}_{n} \in P(n, m)$ (here $\mathbf{0}_{n}$ denotes the empty partial injection), it is also the bottom element of $P(n, m)$. Next,

$$
\nabla_{(n, m)}=(\underbrace{m, m-1, m-2, \ldots}_{n})= \begin{cases}(\underbrace{(\underbrace{m, m-1, m-2, \ldots 1}_{m} \underbrace{0,0, \ldots, 0}_{n-m})}_{m} & \text { if } m \leqslant n \\ (\underbrace{m, m-1, m-2, \ldots m-(n-1)}_{n}) & \text { if } n \leqslant m\end{cases}
$$

is the top element of $\left(P(n, m), \leqslant_{P}\right)$.

Take $n \leqslant m$. Then $\nabla_{(n, m)} \in I(n, m)$, so $\nabla_{(n, m)}$ is also the top element of $\left(I(n, m), \leqslant_{I}\right)$. Next, $\Delta_{(n, m)}=(1,2,3, \ldots, n) \in I(n, m)$ is the bottom element of $\left(I(n, m), \leqslant_{I}\right)$. In particular, $\nabla_{(n)}=\nabla_{(n, n)}$ and the identity function $\Delta_{(n)}=\Delta_{(n, n)}$ are the top and the bottom element of $\left(S(n), \leqslant_{B}\right)$, respectively.

\subsection{Self-duality of posets $\left(F(n, m), \leqslant_{F}\right),\left(T(n, m), \leqslant_{F}\right)$ and $\left(I(n, m), \leqslant_{F}\right)$}

The following result shows that the poset $\left(F(n, m), \leqslant_{F}\right)$ is self-dual for all $n, m \in \mathbb{N}^{*}$. Recall, a function $f: A \longrightarrow A$ is an involution if $f \circ f=i d_{A}$.

Proposition 2. Let $\Phi: F(n, m) \longrightarrow F(n, m)$ be a function such that

$$
\Phi(f)=\mathbf{m}_{n}-f=(m-f(1), m-f(2), \ldots, m-f(n)) \text { for all } f \in F(n, m)
$$

Then $\Phi$ is an involutive anti-automorphism of $F(n, m)$. In particular, $\left(F(n, m), \leqslant_{F}\right)$ is a self-dual poset. Moreover, $\left(\operatorname{Jir}(F(n, m)), \leqslant_{F}\right)$ and $\left(\operatorname{Mir}(F(n, m)), \leqslant_{F}\right)$ are isomorphic to $\left(\operatorname{Mir}(F(n, m)), \leqslant_{F}^{d}\right)$ and $\left(\operatorname{Jir}(F(n, m)), \leqslant_{F}^{d}\right)$, respectively, and these isomorphisms are given by functions $\Phi_{\lceil\operatorname{Jir}(F(n, m))}$ and $\Phi_{\lceil\operatorname{Mir}(F(n, m))}$. In particular, for each $g \in F(n, m), g$ is join-irreducible (respectively, meet-irreducible) iff $\mathbf{m}_{n}-g$ is meet-irreducible (respectively, join-irreducible).

Proof. It is easily shown that if $f \rightarrow g$, then $\Phi(g) \rightarrow \Phi(f)$. Next, since $\Phi$ is an involution, we have that $\Phi$ is bijective and $\Phi(g) \rightarrow \Phi(f)$ implies $f=\Phi(\Phi(f)) \rightarrow \Phi(\Phi(g))=g$.

Take the poset $\left(T(n, m), \leqslant_{F}\right)$, where $m \geqslant 2$, the natural isomorphism $\Theta: F(n, m-$ $1) \longrightarrow T(n, m)$ defined in the previous subsection (i.e., $\Theta(f)=f+\mathbf{1}_{n}$ ) and the antiautomorphism $\Phi$ of $F(n, m)$ introduced in Proposition 2. Then $\Psi=\Theta \circ \Phi \circ \Theta^{-1}$ is an anti-automorphism of $T(n, m)$ such that $\Psi(f)=(\mathbf{m}-\mathbf{1})_{n}-\left(f-\mathbf{1}_{n}\right)+\mathbf{1}_{n}=\mathbf{m}_{n}-f+\mathbf{1}_{n}$ and $\Psi \circ \Psi=\Theta \circ \Phi^{2} \circ \Theta^{-1}=\Theta \circ i d_{F(n, m)} \circ \Theta^{-1}=i d_{T(n, m)}$. Next, $\Psi(I(n, m)) \subseteq I(n, m)$, so $\Psi(I(n, m))=I(n, m)$. Summarizing, since $T(n, 1)=\left\{\mathbf{1}_{n}\right\}$, we obtain that for all $n, m \in \mathbb{N}^{*}$ the following fact holds (of course, it can be also proved directly in a similar way as Proposition 2). 
Corollary 3. Let $\Psi: T(n, m) \longrightarrow T(n, m)$ be a function such that $\Psi(f)=\mathbf{m}_{n}-f+\mathbf{1}_{n}$ for all $f \in T(n, m)$. Then

(a) $\Psi$ is an involutive anti-automorphism of $T(n, m)$. In particular, $\left(T(n, m) \leqslant_{F}\right)$ is self-dual. Moreover, $\left(\operatorname{Jir}(T(n, m)), \leqslant_{F}\right)$ and $\left(\operatorname{Mir}(T(n, m)), \leqslant_{F}\right)$ are isomorphic to $\left(\operatorname{Mir}(T(n, m)), \leqslant_{F}^{d}\right)$ and $\left(\operatorname{Jir}(I(n, m)), \leqslant_{F}^{d}\right)$, respectively, and these isomorphisms are give by functions $\Psi_{\lceil\operatorname{Jir}(T(n, m))}$ and $\Psi_{\lceil\operatorname{Mir}(T(n, m))}$. In particular, for each $g \in$ $T(n, m), g$ is join-irreducible (respectively, meet-irreducible) iff $\mathbf{m}_{n}-g+\mathbf{1}_{n}$ is meetirreducible (respectively, join-irreducible).

(b) If $n \leqslant m$, then $\Psi_{\mid I(n, m)}$ is an involutive anti-automorphism of $I(n, m)$. In particular, $\left(I(n, m) \leqslant_{F}\right)$ is self-dual. Moreover, $\left(\operatorname{Jir}(I(n, m)), \leqslant_{F}\right)$ and $\left(\operatorname{Mir}(I(n, m)), \leqslant_{F}\right)$ are isomorphic to $\left(\operatorname{Mir}(I(n, m)), \leqslant_{F}^{d}\right)$ and $\left(\operatorname{Jir}(I(n, m)), \leqslant_{F}^{d}\right)$, respectively, and these isomorphisms are give by functions $\Psi_{\lceil\operatorname{Jir}(I(n, m))}$ and $\Psi_{\lceil M i r(I(n, m))}$. In particular, for each $g \in I(n, m), g$ is join-irreducible (respectively, meet-irreducible) iff $\mathbf{m}_{n}-g+\mathbf{1}_{n}$ is meet-irreducible (respectively, join-irreducible).

The example of the poset $\left(P(2), \leqslant_{F}\right)$ shows that $\left(P(n, m), \leqslant_{F}\right)$ need not be self-dual. Taking $n=m$ in the point (b) of Corollary 3 we obtain analogous facts for the poset $(S(n) \leqslant B)$.

\section{Lattices of matrices}

M. Fortin has shown in [7] that each partial injective function $f \in P(n)$ can be represented by a square matrix $M(f)$ of size $n$ with non-negative entries. Moreover, he has proved that the function $f \longmapsto M(f)$ is an order embedding of the poset $\left(P(n), \leqslant_{F}\right)$ into the family of all square matrices of size $n$ with non-negative entries and with the natural componentwise order. In this section, using simple facts and methods of linear algebra (e.g., classical operations on rows and columns of matrices), we first give simpler and shorter proofs of these results in a more general case of the poset $F(n, m)$. Thus posets of finite functions $\left(F(n, m), \leqslant_{F}\right),\left(P(n, m), \leqslant_{F}\right),\left(T(n, m), \leqslant_{F}\right)$ and $\left(I(n, m), \leqslant_{F}\right)$ are (up to isomorphism) subposets of the family of matrices of size $n \times m$ with non-negative entries and with the natural componentwise order. Hence, using ideas from [7], we are able to define four lattices of matrices, which contain our posets of finite functions. Next, we show some properties of these lattices. For example, three of them are self-dual. We also show that these lattices have the same numbers of elements as families of row-alternating matrices, alternating matrices and row-alternating sign matrices, respectively. In the next section we will prove that these lattices represent Dedekind-MacNeille completions of $\left(F(n, m), \leqslant_{F}\right),\left(P(n, m), \leqslant_{P}\right),\left(I(n, m), \leqslant_{I}\right)$ and $\left(T(n, m), \leqslant_{T}\right)$. Hence we will obtain additional informations about our posets of finite functions. For instance, their subposets containing all join-irreducible and meet-irreducible elements will be described. 


\subsection{Lattices $\left(M_{n}^{m}(\mathbb{Z}), \leqslant_{M}\right),\left(M_{n}^{m}(\mathbb{N}), \leqslant_{M}\right)$ and $\left(M_{n}^{m}(k), \leqslant_{M}\right)$}

As usual, a matrix $A$ is called an $n \times m$-matrix or equivalently, a matrix of size $n \times m$, if $A$ has $n$ rows and $m$ columns, i.e.,

$$
A=[A[i, j]]_{i=1,2, \ldots n, j=1,2, \ldots m}=\left[\begin{array}{cccc}
A[1,1] & A[1,2] & \ldots & A[1, m] \\
A[2,1] & A[2,2] & \ldots & A[2, m] \\
\vdots & \vdots & \ddots & \vdots \\
A[n, 1] & A[n, 2] & \ldots & A[n, m]
\end{array}\right]
$$

where $n, m \in \mathbb{N}^{*}$ are positive integers.

For each subset $X \subseteq \mathbb{Z}$ (where $\mathbb{Z}$ is the set of all integers), let $M_{n}^{m}(X)$ be a family of all $n \times m$-matrices $A$ such that $A[i, j] \in X$. For all $k \in \mathbb{N}$, let $M_{n}^{m}(k)=M_{n}^{m}(\{0,1, \ldots, k\})$, of course, $M_{n}^{m}(0)$ contains only the zero matrix. Then we have $M_{n}^{m}(k) \subseteq M_{n}^{m}(k+1)$ and $M_{n}^{m}(k) \subseteq M_{n}^{m}(\mathbb{N}) \subseteq M_{n}^{m}(\mathbb{Z})$.

The family $M_{n}^{m}(\mathbb{Z})$ can be partially ordered by the natural componentwise order $\leqslant_{M}$ (see [7]), i.e., for each $A, B \in M_{n}^{m}(\mathbb{Z})$,

$$
A \leqslant_{M} B \text { iff } A[i, j] \leqslant B[i, j] \text { for all } i=1,2, \ldots n, j=1,2, \ldots m .
$$

Obviously $\left(M_{n}^{m}(\mathbb{Z}), \leqslant_{M}\right)$ is isomorphic to the direct power $\mathbb{Z}^{n \cdot m}$ with the product order $\leqslant$ prod. Thus

Proposition 4. $\left(M_{n}^{m}(\mathbb{Z}), \leqslant_{M}\right)$ is a distributive lattice, $\left(M_{n}^{m}(\mathbb{N}), \leqslant_{M}\right)$ is a sublattice of $\left(M_{n}^{m}(\mathbb{Z}), \leqslant_{M}\right)$ and $\left(M_{n}^{m}(k), \leqslant_{M}\right)$ is a finite sublattice of $\left(M_{n}^{m}(\mathbb{N}), \leqslant_{M}\right)$. In particular, $\left(M_{n}^{m}(k), \leqslant_{M}\right)$ is a finite distributive lattice. Moreover, operations of supremum $\vee$ and infimum $\wedge$ are defined as follows: for each $A, B \in M_{n}^{m}(\mathbb{Z})$ and $i=1,2, \ldots n, j=1,2, \ldots m$,

$$
(A \vee B)[i, j]=\max \{A[i, j], B[i, j]\} \text { and }(A \wedge B)[i, j]=\min \{A[i, j], B[i, j]\} .
$$

Lattices $\left(M_{n}^{m}(\mathbb{Z}), \leqslant_{M}\right)$ and $\left(M_{n}^{m}(\mathbb{N}), \leqslant_{M}\right)$ have no top elements, in particular, they are not complete lattices. The first of them has no bottom element, too. But the zero matrix

$$
\mathbf{0}_{n}^{m}=\left[\begin{array}{cccc}
0 & 0 & \cdots & 0 \\
0 & 0 & \cdots & 0 \\
\vdots & \vdots & \cdots & \vdots \\
0 & 0 & \cdots & 0
\end{array}\right] \in M_{n}^{m}(\mathbb{N})
$$

is the bottom element of $\left(M_{n}^{m}(\mathbb{N}), \leqslant_{M}\right)$. Hence $\mathbf{0}_{n}^{m}$ is also the bottom element of the lattice $\left(M_{n}^{m}(k), \leqslant_{M}\right)$ for all $k \in \mathbb{N}$. Next,

$$
\mathbf{k}_{n}^{m}=\left[\begin{array}{cccc}
k & k & \cdots & k \\
k & k & \cdots & k \\
\vdots & \vdots & \cdots & \vdots \\
k & k & \cdots & k
\end{array}\right] \in M_{n}^{m}(k)
$$

is the top element of $\left(M_{n}^{m}(k), \leqslant_{M}\right)$. 


\subsection{Matrix representation}

It is the classical result that each permutation $f \in S(n)$ can be represented by the linear operator $\varphi_{f}: \mathbb{R}^{n} \longrightarrow \mathbb{R}^{n}$ such that $\varphi_{f}\left(\varepsilon_{i}\right)=\varepsilon_{f(i)}$ for $i=1,2, \ldots, n$ and its matrix $M_{\mathcal{S} t}^{\mathcal{S t}}\left(\varphi_{f}\right)=\left[\varphi_{f}\left(\varepsilon_{1}^{n}\right), \varphi_{f}\left(\varepsilon_{2}^{n}\right), \ldots, \varphi_{f}\left(\varepsilon_{n}^{n}\right)\right]$ (here $\varphi_{f}\left(\varepsilon_{1}^{n}\right), \varphi_{f}\left(\varepsilon_{2}^{n}\right), \ldots, \varphi_{f}\left(\varepsilon_{n}^{n}\right)$ are successive columns of the matrix), where $\mathcal{S} t: \varepsilon_{1}^{n}=(1,0 \ldots, 0), \varepsilon_{2}^{n}=(0,1 \ldots, 0), \ldots, \varepsilon_{n}^{n}=(0,0 \ldots, n)$ is the standard base of $\mathbb{R}^{n}$. In fact, it is a faithful group representation of $S(n)$ in the special linear group $S L(n, \mathbb{R})$. In a similar way we can represent functions of $F(n, m)$.

For each $f \in F(n, m)$ take the linear operator $\varphi_{f}: \mathbb{R}^{n} \longrightarrow \mathbb{R}^{m}$ specified by conditions $\varphi_{f}\left(\varepsilon_{i}^{n}\right)=\varepsilon_{f(i)}^{m}$ if $f(i) \neq 0$, and $\varphi_{f}\left(\varepsilon_{i}^{n}\right)=(0,0, \ldots, 0)$ if $f(i)=0$. Let

$$
C(f)=\left[\begin{array}{ccc}
C(f)[1,1] & \cdots & C(f)[1, m] \\
C(f)[2,1] & \cdots & C(f)[2, m] \\
\vdots & \ddots & \vdots \\
C(f)[n, 1] & \cdots & C(f)[n, m]
\end{array}\right]
$$

be the transposition of the matrix $M_{\mathcal{S} t}^{\mathcal{S} t}\left(\varphi_{f}\right)$ corresponding to $\varphi_{f}$, i.e., $C(f)=\left(M_{\mathcal{S} t}^{\mathcal{S t}}\left(\varphi_{f}\right)\right)^{T}$. Equivalently,

$$
C(f)[i, j]=\left\{\begin{array}{ll}
1 & \text { if } j=f(i) \\
0 & \text { if } j \neq f(i)
\end{array} .\right.
$$

Of course, this matrix uniquely determines $f$.

Let $M^{c}(f)$ and $M^{r}(f)$ be matrices obtained from $C(f)$ in the following way:

$$
\begin{gathered}
M^{c}(f)[i, j]=C(f)[i, j]+C(f)[i, j+1]+\cdots+C(f)[i, m], \\
M^{r}(f)[i, j]=C(f)[1, j]+C(f)[2, j]+\cdots+C(f)[i, j],
\end{gathered}
$$

i.e., the $j$-th column of $M^{c}(f)$ is the sum of columns of $C(f)$ from the $j$-th to the $m$-th, and similarly, the $i$-th row of $M^{r}(f)$ is the sum of rows of $C(f)$ from the first to the $i$-th.

Of course,

$$
M^{c}(f)[i, j]=\left\{\begin{array}{ll}
1 & \text { if } j \leqslant f(i) \\
0 & \text { if } j>f(i)
\end{array} .\right.
$$

Now we can introduce the following definition.

Definition 5. For each $f \in F(n, m)$, let $M(f)$ be an $n \times m$-matrix such that

$$
M(f)[i, j]=\sum_{k=1}^{i} M^{c}(f)[k, j]=\sum_{k=j}^{m} M^{r}(f)[i, k]=\sum_{k \leqslant i, l \geqslant j} C[k, l],
$$

i.e., the $i$-th row of $M(f)$ is the sum of the rows of $M^{c}(f)$ from the first to the $i$-th, the $j$-th column of $M(f)$ is the sum of the columns of $M^{r}(f)$ from the $j$-th to the $m$-th, and $M(f)[i, j]$ is the sum of all entries of $C(f)$ which lie above and to the right of the place $(i, j)$. 
For $n=m$ and $f \in P(n)$, our definition of the matrix $M(f)$ is equivalent with the definition formulated in [7], Section 3.2. Therefore we use the same notation.

For example, take $f=\left(\begin{array}{llll}3 & 0 & 2 & 4\end{array}\right) \in P(5)$. Then (see also [7], Example 3.3) matrices $C(f), M^{c}(f), M^{r}(f)$ and $M(f)$ are equal, respectively, to

$$
\left[\begin{array}{lllll}
0 & 0 & 1 & 0 & 0 \\
0 & 0 & 0 & 0 & 0 \\
0 & 1 & 0 & 0 & 0 \\
0 & 0 & 0 & 1 & 0 \\
1 & 0 & 0 & 0 & 0
\end{array}\right],\left[\begin{array}{lllll}
1 & 1 & 1 & 0 & 0 \\
0 & 0 & 0 & 0 & 0 \\
1 & 1 & 0 & 0 & 0 \\
1 & 1 & 1 & 1 & 0 \\
1 & 0 & 0 & 0 & 0
\end{array}\right],\left[\begin{array}{lllll}
0 & 0 & 1 & 0 & 0 \\
0 & 0 & 1 & 0 & 0 \\
0 & 1 & 1 & 0 & 0 \\
0 & 1 & 1 & 1 & 0 \\
1 & 1 & 1 & 1 & 0
\end{array}\right],\left[\begin{array}{lllll}
1 & 1 & 1 & 0 & 0 \\
1 & 1 & 1 & 0 & 0 \\
2 & 2 & 1 & 0 & 0 \\
3 & 3 & 2 & 1 & 0 \\
4 & 3 & 2 & 1 & 0
\end{array}\right] .
$$

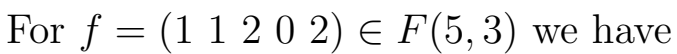

$$
C(f)=\left[\begin{array}{lll}
1 & 0 & 0 \\
1 & 0 & 0 \\
0 & 1 & 0 \\
0 & 0 & 0 \\
0 & 1 & 0
\end{array}\right], M^{c}(f)=\left[\begin{array}{lll}
1 & 0 & 0 \\
1 & 0 & 0 \\
1 & 1 & 0 \\
0 & 0 & 0 \\
1 & 1 & 0
\end{array}\right], M^{r}(f)=\left[\begin{array}{lll}
1 & 0 & 0 \\
2 & 0 & 0 \\
2 & 1 & 0 \\
2 & 1 & 0 \\
2 & 2 & 0
\end{array}\right], M(f)=\left[\begin{array}{lll}
1 & 0 & 0 \\
2 & 0 & 0 \\
3 & 1 & 0 \\
3 & 1 & 0 \\
4 & 2 & 0
\end{array}\right] .
$$

For each $f \in F(n, m)$ we have $C(f), M^{c}(f) \in M_{n}^{m}(1)$ and $M^{r}(f), M(f) \in M_{n}^{m}(n)$. Moreover, $f \in P(n, m)$ iff $M^{r}(f) \in M_{n}^{m}(1)$; and $f \in T(n, m)$ iff the first column of $M^{c}(f)$ equals $\left[\begin{array}{lllll}1 & 1 & 1 & \ldots & 1\end{array}\right]^{T}$ iff the first column of $M(f)$ equals $\left[\begin{array}{lllll}1 & 2 & 3 & \ldots & n\end{array}\right]^{T}$ iff $M(f)[n, 1]=n$.

Operations on rows and columns of $C(f)$ are invertible, so $M^{c}(f)$ and $M^{r}(f)$ can be rebuild from $M(f)$, and similarly, $C(f)$ can be rebuild from $M^{c}(f)$ and also from $M^{r}(f)$. For instance, $M^{c}(f)[i, j]=M(f)[i, j]-M(f)[i-1, j]$ and $C(f)[i, j]=M^{c}(f)[i, j]-$ $M^{c}(f)[i, j+1]$, where $M(f)[0, j]=0$ and $M^{c}(f)[i, m+1]=0$. Hence, for all $f, g \in F(n, m)$,

$$
M(f)=M(g) \text { implies } f=g .
$$

In particular, the function $f \longmapsto M(f)$ is injective.

Now we show a more general result. This result is proved by M. Fortin in [7] only for $P(n)$, but his proof can be adopted without major changes for $F(n, m)$. However, we now formulate another simpler and shorter proof of this fact.

Theorem 6. The function $f \longmapsto M(f)$ is an embedding of the poset $\left(F(n, m), \leqslant_{F}\right)$ into $\left(M_{n}^{m}(n), \leqslant_{M}\right)$, i.e., for all $f, g \in F(n, m), f \leqslant_{F} g$ iff $M(f) \leqslant_{M} M(g)$.

Proof. $\Longrightarrow$ : Take $f, g \in F(n, m)$. If $f \leqslant$ prod $g$, then $M^{c}(f) \leqslant{ }_{M} M^{c}(g)$, so also $M(f) \leqslant M$ $M(g)$. If $f \rightarrow_{B} g$, then there are $i_{1}<i_{2}, j_{1}<j_{2}$ such that $C(f)\left[i_{1}, j_{1}\right]=1, C(f)\left[i_{1}, j_{2}\right]=0$, $C(f)\left[i_{2}, j_{1}\right]=0, C(f)\left[i_{2}, j_{2}\right]=1, C(g)\left[i_{1}, j_{1}\right]=0, C(g)\left[i_{1}, j_{2}\right]=1, C(g)\left[i_{2}, j_{1}\right]=1$, $C(g)\left[i_{2}, j_{2}\right]=0$ and $C(f)[i, j]=C(g)[i, j]$ for all other $i, j$. So we have $M(f)[i, j]=$ $\sum_{k \leqslant i, l \geqslant j} C(f)[k, l] \leqslant \sum_{k \leqslant i, l \geqslant j} C(g)[k, l]=M(g)$ for all $i, j$. By these two facts we obtain our implication, because $\leqslant_{F}$ is the transitive closure of $\leqslant_{\text {prod }} \cup \rightarrow_{B}$.

$\Longleftarrow$ : Take $f, g \in F(n, m)$ such that $M(f) \leqslant_{M} M(g)$. Since the function $f \longmapsto M(f)$ is injective, we can assume that $M(f) \neq M(g)$. Then it is sufficient to show (see [7]) that there is $f^{\prime} \in F(n, m)$ such that $f ł_{F} f^{\prime}$ and $M\left(f^{\prime}\right) \leqslant_{M} M(g)$. If $f \leqslant_{\text {prod }} g$, then $f \leqslant_{F} g$. Thus assume $f \Varangle_{\text {prod }} g$ and take the least number $1 \leqslant i_{0} \leqslant n$ such that $f\left(i_{0}\right)>g\left(i_{0}\right)$. Since $M^{c}(f)[1, j]=M(f)[1, j] \leqslant M(g)[1, j]=M^{c}(g)[1, j]$, we have $i_{0} \geqslant 2$. 
Let $A$ be the set of all numbers $1 \leqslant l<i_{0}$ such that $f\left(i_{0}\right) \leqslant f(l)$. Then we have $M^{c}(f)\left[l, f\left(i_{0}\right)\right]=1$ for $l \in A \cup\left\{i_{0}\right\}$, so $M(g)\left[i_{0}, f\left(i_{0}\right)\right] \geqslant M(f)\left[i_{0}, f\left(i_{0}\right)\right]=M^{c}(f)\left[1, f\left(i_{0}\right)\right]+$ $M^{c}(f)\left[2, f\left(i_{0}\right)\right]+\cdots+M^{c}(f)\left[i_{0}, f\left(i_{0}\right)\right]=|A|+1$. Hence

$$
M^{c}(g)\left[1, f\left(i_{0}\right)\right]+M^{c}(g)\left[2, f\left(i_{0}\right)\right]+\cdots+M^{c}(g)\left[i_{0}, f\left(i_{0}\right)\right] \geqslant|A|+1 .
$$

Next, $M^{c}(g)\left[i_{0}, f\left(i_{0}\right)\right]=0\left(\right.$ since $\left.f\left(i_{0}\right)>g\left(i_{0}\right)\right)$ and for each $1 \leqslant i<i_{0}, M^{c}(f)\left[i, f\left(i_{0}\right)\right]=1$ implies $M^{c}(g)\left[i, f\left(i_{0}\right)\right]=1$ (because $f(i) \leqslant g(i)$ for $1 \leqslant i<i_{0}$ ). By these three facts, there is $1 \leqslant k_{0}<i_{0}$ such that

$$
M^{c}(g)\left[k_{0}, f\left(i_{0}\right)\right]=1 \quad \text { and } \quad M^{c}(f)\left[k_{0}, f\left(i_{0}\right)\right]=0,
$$

so $f\left(i_{0}\right) \leqslant g\left(k_{0}\right)$ and $f\left(k_{0}\right)<f\left(i_{0}\right)$.

Let $f^{\prime} \in F(n, m)$ be a function such that $f^{\prime}\left(k_{0}\right)=f\left(i_{0}\right), f^{\prime}\left(i_{0}\right)=f\left(k_{0}\right)$ and $f^{\prime}(i)=f(i)$ for $i \neq k_{0}, i_{0}$. Then $f \rightarrow_{B} f^{\prime}$. Next, for $s=f\left(k_{0}\right)+1, f\left(k_{0}\right)+2, \ldots, f\left(i_{0}\right)$,

$M^{c}\left(f^{\prime}\right)\left[k_{0}, s\right]=1=M^{c}(f)\left[k_{0}, s\right]+1=M^{c}(g)\left[k_{0}, s\right], \quad M^{c}\left(f^{\prime}\right)\left[i_{0}, s\right]=0=M^{c}(f)\left[i_{0}, s\right]-1$

and for all other pairs $(l, j)$,

$$
M^{c}\left(f^{\prime}\right)[l, j]=M^{c}(f)[l, j] .
$$

Since $f(i) \leqslant g(i)$ for $i<i_{0}$, we have also that

$$
M^{c}(f)[l, j] \leqslant M^{c}(g)[l, j] \text { for } l=1,2, \ldots, i_{0}-1 \text { and } 1 \leqslant j \leqslant m .
$$

Now take $1 \leqslant r \leqslant n$ and $1 \leqslant s \leqslant m$. Then by the above facts we obtain:

If $s \notin\left\{f\left(k_{0}\right)+1, f\left(k_{0}\right)+2, \ldots, f\left(i_{0}\right)\right\}$ or $1 \leqslant r \leqslant k_{0}-1$, then $M\left(f^{\prime}\right)[r, s]=$ $M^{c}\left(f^{\prime}\right)[1, s]+M^{c}\left(f^{\prime}\right)[2, s]+\cdots+M^{c}\left(f^{\prime}\right)[r, s]=M^{c}(f)[1, s]+M^{c}(f)[2, s]+\cdots+M^{c}(f)[r, s]=$ $M(f)[r, s] \leqslant M(g)[r, s]$.

If $k_{0} \leqslant r \leqslant i_{0}-1$ and $s \in\left\{f\left(k_{0}\right)+1, f\left(k_{0}\right)+2, \ldots, f\left(i_{0}\right)\right\}$, then $M\left(f^{\prime}\right)[r, s]=$ $M^{c}\left(f^{\prime}\right)[1, s]+\cdots+M^{c}\left(f^{\prime}\right)\left[k_{0}, s\right]+\cdots+M^{c}\left(f^{\prime}\right)[r, s]=M^{c}(f)[1, s]+\cdots+\left(M^{c}(f)\left[k_{0}, s\right]+\right.$ $1)+\cdots+M^{c}(f)[r, s] \leqslant M^{c}(g)[1, s]+\cdots+M^{c}(g)\left[k_{0}, s\right]+\cdots+M^{c}(g)[r, s]=M(g)[r, s]$.

If $i_{0} \leqslant r \leqslant n$ and $s \in\left\{f\left(k_{0}\right)+1, f\left(k_{0}\right)+2, \ldots, f\left(i_{0}\right)\right\}$, then $M\left(f^{\prime}\right)[r, s]=M^{c}\left(f^{\prime}\right)[1, s]+$ $\cdots+M^{c}\left(f^{\prime}\right)\left[k_{0}, s\right]+\cdots+M^{c}\left(f^{\prime}\right)\left[i_{0}, s\right]+\cdots+M^{c}\left(f^{\prime}\right)[r, s]=M^{c}(f)[1, s]+\cdots+\left(M^{c}(f)\left[k_{0}, s\right]+\right.$ 1) $+\cdots+\left(M^{c}(f)\left[i_{0}, s\right]-1\right)+\cdots+M^{c}(f)[r, s]=M(f)[r, s] \leqslant M(g)[r, s]$.

Definition 7. For all $n, m \in \mathbb{N}^{*}$, let $F_{n}^{m}=\{M(f): f \in F(n, m)\}, P_{n}^{m}=\{M(f): f \in$ $P(n, m)\}, T_{n}^{m}=\{M(f): f \in T(n, m)\}$ and $I_{n}^{m}=\{M(f): f \in I(n, m)\}$. Moreover, $S_{n}^{n}=I_{n}^{n}=\{M(f): f \in S(n)\}$.

Note that $P_{n}^{n}$ is denoted by $R_{n}$ in [7]. Of course, $I_{n}^{m} \subseteq T_{n}^{m} \subseteq F_{n}^{m}$ and $I_{n}^{m} \subseteq P_{n}^{m} \subseteq F_{n}^{m}$. If $n>m$, then $I_{n}^{m}=\emptyset$. Recall that the function $f \longmapsto M(f)$ is injective. So $I_{n}^{m}=$ $P_{n}^{m} \cap T_{n}^{m}$. Moreover, we can introduce the following notation (which will be used in the third section).

Remark 8. For each matrix $A \in F_{n}^{m}$, there is an exactly one function of $F(n, m)$, denoted by $f_{A}$, such that $M\left(f_{A}\right)=A$. 
Sets $F_{n}^{m}, P_{n}^{m}, T_{n}^{m}, I_{n}^{m}$ induce subposets of $\left(M_{n}^{m}(n), \leqslant_{M}\right)$, which are isomorphic to $\left(F(n, m), \leqslant_{F}\right),\left(P(n, m), \leqslant_{P}\right),\left(T(n, m), \leqslant_{P}\right),\left(I(n, m), \leqslant_{I}\right)$, respectively, by Theorem 6 . In particular, $\left(S(n), \leqslant_{B}\right)$ is isomorphic to $\left(S_{n}^{n}, \leqslant_{M}\right)$.

For all $m \in \mathbb{N}^{*}$,

$$
I_{1}^{m}=T_{1}^{m}=\left\{\left[\begin{array}{llllll}
1 & 0 & 0 & \ldots & 0 & 0
\end{array}\right],\left[\begin{array}{llllll}
1 & 1 & 0 & \ldots & 0 & 0
\end{array}\right], \ldots,\left[\begin{array}{llllll}
1 & 1 & 1 & \ldots & 1 & 0
\end{array}\right],\left[\begin{array}{llllll}
1 & 1 & 1 & \ldots & 1 & 1
\end{array}\right]\right\}
$$

is an $m$-element chain and $P_{1}^{m}=F_{1}^{m}=\left\{\left[\begin{array}{llllll}0 & 0 & 0 & \ldots & 0 & 0\end{array}\right]\right\} \cup T_{1}^{m}$ is an $m+1$-element chain. Next, for each $n \in \mathbb{N}^{*}$,

$P_{n}^{1}=\left\{\left[\begin{array}{llllll}0 & 0 & \ldots & 0 & 0 & 0\end{array}\right]^{T},\left[\begin{array}{llllll}0 & 0 & \ldots & 0 & 0 & 1\end{array}\right]^{T},\left[\begin{array}{lllll}0 & 0 & \ldots & 0 & 1\end{array}\right]^{T}, \ldots\left[\begin{array}{llllll}0 & 1 & \ldots & 1 & 1 & 1\end{array}\right]^{T},\left[\begin{array}{llllll}1 & 1 & \ldots & 1 & 1 & 1\end{array}\right]^{T}\right\}$

is an $n+1$-element chain (hence we obtain another proof of the fact that $\left(P(n, 1), \leqslant_{F}\right)$ is an $n+1$-element chain).

Finally, take posets $T(n, m)$ and $T_{n}^{m}$. If $m=1$, then $T(n, 1)=\left\{\left(\begin{array}{llll}1 & 1 & 1 & \ldots\end{array}\right)\right\}$ and $\left.T_{n}^{1}=\left\{\begin{array}{lllll}1 & 2 & 3 & \ldots & n\end{array}\right]^{T}\right\}$. Thus assume that $m \geqslant 2$. Then we know that the function $f \longmapsto f-\mathbf{1}_{n}$ is an isomorphism of $\left(T(n, m), \leqslant_{F}\right)$ onto $\left(F(n, m-1), \leqslant_{F}\right)$ and the function $g \longmapsto g+\mathbf{1}_{n}$ is its inverse. It is easy to see that $M\left(f-\mathbf{1}_{n}\right)=\downarrow M(f)$ for each $f \in T(n, m)$, where $\downarrow A$ is the $n \times(m-1)$-matrix obtained from an $n \times m$-matrix $A$ by removing its first column. Similarly, we obtain $M\left(g+\mathbf{1}_{n}\right)=\uparrow M(g)$ for each $g \in F(n, m-1)$, where $\uparrow A$ be an $n \times(k+1)$-matrix obtained from an $n \times k$-matrix $A$ by adding the first column $\left[\begin{array}{lllll}1 & 2 & 3 & \ldots & n\end{array}\right]^{T}$. Hence the function $\Phi: A \longmapsto \downarrow A$ is an isomorphism of $\left(T_{n}^{m}, \leqslant_{M}\right)$ onto $\left(F_{n}^{m-1}, \leqslant_{M}\right)$, and $\Psi: A \longmapsto \uparrow A$ is an isomorphism of $\left(F_{n}^{m-1}, \leqslant_{M}\right)$ onto $\left(T_{n}^{m}, \leqslant_{M}\right)$. Moreover, it is obvious that $\downarrow \uparrow A=A$ for each matrix $A$, and it is not difficult to see that $\uparrow \downarrow A=A$ for each $A \in T_{n}^{m}$. Thus $\Psi$ is the inverse function of $\Phi$.

\subsection{Special sublattices of $\left(M_{n}^{m}(n), \leqslant_{M}\right)$}

Posets $\left(F_{n}^{m}, \leqslant_{M}\right),\left(P_{n}^{m}, \leqslant_{M}\right)\left(T_{n}^{m}, \leqslant_{M}\right)$, and $\left(I_{n}^{m}, \leqslant_{M}\right)$ are not lattices. Therefore now, applying methods from [7], we define families $F G_{n}^{m}, P G_{n}^{m} T G_{n}^{m}$, and $I G_{n}^{m}$ of matrices, which contains these posets and form sublattices of $\left(M_{n}^{m}(n), \leqslant_{M}\right)$. In fact we will show in the next section that these lattices are (up to isomorphism) Dedekind-MacNeille completions of these posets, and consequently, of posets $\left(F(n, m), \leqslant_{F}\right),\left(P(n, m), \leqslant_{F}\right),\left(T(n, m), \leqslant_{F}\right)$ and $\left(I(n, m), \leqslant_{F}\right)$.

Definition 9. Let $n, m \in \mathbb{N}^{*}$ be positive integers. Then

(a) $F G_{n}^{m}$ is a set of all $n \times m$-matrices $A \in M_{n}^{m}(\mathbb{N})$ such that

(1) the columns of $A$, from the top to the bottom, are increasing and starting by 0 or 1 ,

(2) each two adjacent numbers on a column of $A$ are equal or differ by 1 ,

(3) the rows of $A$, from the left to the right, are decreasing.

(b) $P G_{n}^{m}$ is a set of all matrices $A \in F G_{n}^{m}$ such that 
(1) the rows of $A$, from the left to the right, are decreasing and ending by 0 or 1 ,

(2) each two adjacent numbers on a row of $A$ are equal or differ by 1 .

(c) $T G_{n}^{m}$ is a set of all matrices $A \in F G_{n}^{m}$ such that $A[n, 1]=n$ (equivalently, the first column of $A$ is equal to $\left[\begin{array}{llll}1 & 2 & \ldots & n\end{array}\right]^{T}$ ).

(d) $I G_{n}^{m}=P G_{n}^{m} \cap T G_{n}^{m}$. In other words, $I G_{n}^{m}$ is a set of all matrices $A \in P G_{n}^{m}$ such that $A[n, 1]=n$ (equivalently, the first column of $A$ is equal to $[1,2, \ldots, n]^{T}$ ).

If $n=m$, then $I G_{n}^{n}$ will be denoted by $S G_{n}^{n}$ (equivalently, $S G_{n}^{n}$ is a set of all matrices $A \in P G_{n}^{n}$ such that the first column of $A$ is equal to $[1,2, \ldots, n]^{T}$ and the last row is equal to $[1,2, \ldots, n])$.

Note that the family $P G_{n}^{n}$ is introduced in [7], where it is denoted by $R G_{n}$. Next, conditions (b.1) and (b.2) imply that $I G_{n}^{m}=\emptyset$ for $n>m$.

For each $m \in \mathbb{N}^{*}, F G_{1}^{m}=F_{1}^{m}, P G_{1}^{m}=P_{1}^{m}, T G_{1}^{m}=T_{1}^{m}$ and $I G_{1}^{m}=I_{1}^{m}$; in particular, $P G_{1}^{m}=F G_{1}^{m}$ and $I G_{1}^{m}=T G_{1}^{m}$. Next, for each $\left.n \in \mathbb{N}^{*}, T G_{n}^{1}=T_{n}^{1}=\left\{\begin{array}{lllll}1 & 2 & 3 & \ldots & n\end{array}\right]^{T}\right\}$ and $P G_{n}^{1}=P_{n}^{1}$.

Conditions (a.1) and (a.2) of Definition 9 imply

$$
0 \leqslant A[i, j] \leqslant i \leqslant n \text { for each } A \in F G_{n}^{m} .
$$

Hence $P G_{n}^{m}, T G_{n}^{m}, I G_{n}^{m} \subseteq F G_{n}^{m} \subseteq M_{n}^{m}(n)$. In particular, all these sets are finite.

By these two inequalities and conditions (b.1), (b.2) of Definition 9 we obtain

$$
0 \leqslant A[i, j] \leqslant \min \{i, m-j+1\} \leqslant \min \{n, m\} \text { for each } A \in P G_{n}^{m} .
$$

In particular, $P G_{n}^{m}, I G_{n}^{m} \subseteq M_{n}^{m}(\min \{n, m\})$.

Conditions (b.1), (b.2) and (d) of Definition 9 imply also

$$
\max \{0, i-j+1\} \leqslant A[i, j] \text { for each } A \in I G_{n}^{m} .
$$

The set $F G_{n}^{m}$ induces a subposet of the lattice $\left(M_{n}^{m}(n), \leqslant_{M}\right)$, and sets $P G_{n}^{m}, T G_{n}^{m}, I G_{n}^{m}$ induce subposets of $\left(F G_{n}^{m}, \leqslant_{M}\right)$. Moreover, it is easy to see that the following more general result holds (it generalizes Theorem 4.16 formulated and proved in [7] for $P G_{n}^{n}$ ).

Proposition 10. Let $n, m \in \mathbb{N}^{*}$. Then

(a) $\left(F G_{n}^{m}, \leqslant_{M}\right)$ is a sublattice of the lattice $\left(M_{n}^{m}(n), \leqslant_{M}\right)$. In particular, it is a finite distributive lattice with the join and meet operations defined as in Proposition 4.

(b) $\left(P G_{n}^{m}, \leqslant_{M}\right),\left(T G_{n}^{m}, \leqslant_{M}\right)$ and $\left(I G_{n}^{m}, \leqslant_{M}\right)$ (if $\left.n \leqslant m\right)$ are sublattices of $\left(F G_{n}^{m}, \leqslant_{M}\right)$.

The lattice $\left(I G_{n}^{m}, \leqslant_{M}\right)$ (if $\left.1 \leqslant n \leqslant m\right)$ is a sublattice of lattices $\left(P G_{n}^{m}, \leqslant_{M}\right)$ and $\left(T G_{n}^{m}, \leqslant_{M}\right)$. Moreover, $\left(P G_{n}^{m}, \leqslant_{M}\right)$ is a sublattice of the lattice $\left(M_{n}^{m}(\min \{n, m\}), \leqslant_{M}\right)$.

For each $m \in \mathbb{N}^{*}, F G_{1}^{m}=P G_{1}^{m}$ is an $m+1$-element chain and $T G_{1}^{m}=I G_{1}^{m}$ is $m$-element chain. Next, for each $n \in \mathbb{N}^{*},\left(P G_{n}^{1}, \leqslant_{M}\right)$ is an $n+1$-element chain. 
Take the lattice $\left(T G_{n}^{m}, \leqslant_{M}\right)$. If $m=1$, then it is a trivial (i.e., one-element) lattice. For $m \geqslant 2$ it is easy to see that the function $\Phi: A \longmapsto \downarrow A$, for all $A \in T G_{n}^{m}$, is an embedding of $\left(T G_{n}^{m}, \leqslant_{M}\right)$ into $\left(F G_{n}^{m-1}, \leqslant_{M}\right)$; recall that $\downarrow A$ is the $n \times(m-1)$-matrix obtained from an $n \times m$-matrix $A$ by removing its first column. Next, observe that if $A \in F G_{n}^{m-1}$, then $\uparrow A \in T G_{n}^{m}$ (rows of $\uparrow A$ are decreasing, because $A[i, 1] \leqslant i$ ), recall that $\uparrow A$ is an $n \times(k+1)$-matrix obtained from an $n \times k$-matrix $A$ by adding the first column $\left[\begin{array}{lllll}1 & 2 & 3 & \ldots & n\end{array}\right]^{T}$. Thus the function $\Psi: A \longmapsto \uparrow A$, for all $A \in F G_{n}^{m-1}$, is an embedding of $\left(F G_{n}^{m-1}, \leqslant_{M}\right)$ into $\left(T G_{n}^{m}, \leqslant_{M}\right)$. Moreover, $\uparrow \downarrow A=A$ for each $A \in T G_{n}^{m}$ and $\downarrow \uparrow A=A$ for each $A \in F G_{n}^{m-1}$. So $\Psi$ is the inverse function of $\Phi$. Hence $\Phi$ is an isomorphism between $\left(T G_{n}^{m}, \leqslant_{M}\right)$ and $\left(F G_{n}^{m-1}, \leqslant_{M}\right)$.

Now we formulate some technical notions which will be needed later.

Definition 11. Let $A \in M_{n}^{m}(\mathbb{Z})$. Then

(a) $O^{r}(A)$ is a matrix such that $O^{r}(A)[i, j]=A[i, j]-A[i-1, j]$, where $A[0, j]=0$, for $i=1,2, \ldots, n, j=1,2, \ldots, m$ (i.e., the $i$-th row of $O^{r}(A)$ is the difference of the $i$-th and the $i-1$-th row of $A$ ).

(b) $O^{c}(A)$ is a matrix such that $O^{c}(A)[i, j]=A[i, j]-A[i, j+1]$, where $A[i, m+1]=0$, for $i=1,2, \ldots, n$ and $j=1,2, \ldots, m$ (i.e., the $j$-th column of $O^{c}(A)$ is the difference of the $j$-th and the $j+1$-th column of $A$ ).

(c) $O(A)=O^{c}\left(O^{r}(A)\right)=O^{r}\left(O^{c}(A)\right)$, i.e., $O(A)[i, j]=(A[i, j]-A[i-1, j])-(A[i, j+$ $1]-A[i-1, j+1])=(A[i, j]+A[i-1, j+1])-(A[i-1, j]+A[i, j+1])$, where $A[0, j]=0$ and $A[i, m+1]=0$.

(d) $A^{\text {ht }}$ is a matrix (called the horizontal transposition of $A$ ) obtained from $A$ by changing the positions of columns from the last to the first, i.e., $A^{h t}[i, j]=A[i, m-j+1]$.

(e) $A^{v t}$ is a matrix (called the vertical transposition of $A$ ) obtained from $A$ by changing the positions of rows from the bottom to the top, i.e., $A^{h t}[i, j]=A[n-i+1, j]$.

(f) $A^{q t}=\left(\left(A^{h t}\right)^{v t}\right)^{T}=\left(\left(A^{v t}\right)^{h t}\right)^{T}$ (where ${ }^{T}$ denotes the transposition of matrix), equivalently, $A^{q t}[i, j]=A[m-j+1, n-i+1]$. This matrix will be called the quasitransposition of $A$. In other words, $A^{q t}$ is obtained from $A$ by the mirror symmetry with respect to the second diagonal (i.e., the diagonal from the left and the bottom to the right and the top).

(g) If $A$ is a $0-1$ matrix, i.e., $A \in M_{n}^{m}(1)$, then $A^{\text {neg }}=\mathbf{1}_{n}^{m}-A$ (i.e., $A^{\text {neg }}[i, j]=$ $1-A[i, j])$, equivalently, $A^{\text {neg }}$ is obtained from $A$ by replacing each zero entry by 1 and each one entry by 0 .

Take $A=\left[\begin{array}{lll}1 & 0 & 0 \\ 1 & 1 & 0 \\ 2 & 2 & 0\end{array}\right]$. Then $A \in F G_{3}^{3}$ and $A \notin P G_{3}^{3}, A \notin T G_{3}^{3}, A \notin I G_{3}^{3}$. Next,

$$
O^{r}(A)=\left[\begin{array}{lll}
1 & 0 & 0 \\
0 & 1 & 0 \\
1 & 1 & 0
\end{array}\right], \quad O^{c}(A)=\left[\begin{array}{lll}
1 & 0 & 0 \\
0 & 1 & 0 \\
0 & 2 & 0
\end{array}\right], \quad O(A)=\left[\begin{array}{ccc}
1 & 0 & 0 \\
-1 & 1 & 0 \\
0 & 1 & 0
\end{array}\right]
$$




$$
A^{h t}=\left[\begin{array}{lll}
0 & 0 & 1 \\
0 & 1 & 1 \\
0 & 2 & 2
\end{array}\right], \quad A^{v t}=\left[\begin{array}{lll}
2 & 2 & 0 \\
1 & 1 & 0 \\
1 & 0 & 0
\end{array}\right], \quad A^{q t}=\left[\begin{array}{lll}
0 & 0 & 0 \\
2 & 1 & 0 \\
2 & 1 & 1
\end{array}\right] .
$$

Take $A \in M_{n}^{m}(\mathbb{Z})$. Then $O(A)^{q t}[i, j]=O(A)[m-j+1, n-i+1]=(A[m-j+$ $1, n-i+1]+A[m-j, n-i+2])-(A[m-j, n-i+1]+A[m-j+1, n-i+2])=$ $\left(A^{q t}[i, j]+A^{q t}[i-1, j+1]\right)-\left(A^{q t}[i, j+1]+A^{q t}[i-1, j]\right)=O\left(A^{q t}\right)[i, j]$. Thus

$$
O\left(A^{q t}\right)=O(A)^{q t}
$$

It is also easy to show that $O(A)=O^{r}\left(O^{r}(A)^{q t}\right)^{q t}=O^{r}\left(O^{r}(A)^{T}\right)^{T}$. Next, $O^{r}\left(A^{h t}\right)=$ $O^{r}(A)^{h t}, O^{c}\left(A^{v t}\right)=O^{c}(A)^{v t},\left(A^{q t}\right)^{q t}=A,\left(A^{h t}\right)^{h t}=A,\left(A^{v t}\right)^{v t}=A$. If $A$ is a $0-1$-matrix, then $\left(A^{\text {neq }}\right)^{h t}=\left(A^{h t}\right)^{n e q},\left(A^{n e q}\right)^{v t}=\left(A^{v t}\right)^{n e q}$ and $\left(A^{\text {neq }}\right)^{q t}=\left(A^{q t}\right)^{n e q}$.

Matrices $O(A), O^{r}(A), O^{c}(A)$ are obtained from $A$ by invertible operations, so $A$ can be rebuild from these matrices, and also $O^{r}(A), O^{c}(A)$ can be rebuild from $O(A)$. More precisely,

$$
\begin{gathered}
O^{r}(A)[i, j]=\sum_{k=j}^{m} O(A)[i, k], O^{c}(A)[i, j]=\sum_{k=1}^{i} O(A)[k, j], \\
A[i, j]=\sum_{k=1}^{i} O^{r}(A)[k, j]=\sum_{k=j}^{m} O^{c}(A)[i, k]=\sum_{k \leqslant i, l \geqslant j} O(A)[k, l] .
\end{gathered}
$$

In particular, for all $A, B \in M_{n}^{m}(\mathbb{Z})$,

$$
\text { if } O(A)=O(B) \text { or } O^{r}(A)=O^{r}(B) \text { or } O^{c}(A)=O^{c}(B) \text {, then } A=B \text {. }
$$

Applying the above results it is easy to see that for every function $f \in F(n, m)$,

$$
O^{r}(M(f))=M^{c}(f), \quad O^{c}(M(f))=M^{r}(f) \text { and } O(M(f))=C(f) .
$$

For each $A \in M_{n}^{m}(\mathbb{N})$ we have also the following simple facts:

$A \in F G_{n}^{m}$ implies that $O^{r}(A)$ is a $0-1$ matrix, i.e., $O^{r}(A) \in M_{n}^{m}(1)$.

$A \in P G_{n}^{m}$ iff $A \in F G_{n}^{m}$ and $A^{q t} \in F G_{m}^{n}$.

$A \in F_{n}^{m}$ iff each row of $O^{r}(A)$ is decreasing from the left to the right, i.e. each row of $O^{r}(A)$ is of the form $[\underbrace{1,1, \ldots, 1}_{k}, 0,0, \ldots, 0]$, where $0 \leqslant k \leqslant m$.

Moreover, if $n=m$, then $A \in S G_{n}^{n}$ iff $A^{q t} \in S G_{n}^{n}$.

\section{Proposition 12.}

(a) $F_{n}^{m} \subseteq F G_{n}^{m}, P_{n}^{m} \subseteq P G_{n}^{m}, T_{n}^{m} \subseteq T G_{n}^{m}$ and $I_{n}^{m} \subseteq I G_{n}^{m}$.

(b) $F_{n}^{m} \cap P G_{n}^{m}=P_{n}^{m}, F_{n}^{m} \cap T G_{n}^{m}=T_{n}^{m}$ and $F_{n}^{m} \cap I G_{n}^{m}=I_{n}^{m}$.

Proof. (a): Take $f \in F(n, m)$. Then $M^{c}(f)$ is a $0-1$ matrix which has decreasing rows, so $M(f) \in F G_{n}^{m}$. If $f \in P(n, m)$, then additionally $M^{r}(f)$ is a $0-1$ matrix, so $M(f) \in P G_{n}^{m}$. If $f \in T(n, m)$, then $M(f)[n, 1]=n$, so $M(f) \in T G_{n}^{m}$. Finally, $I_{n}^{m}=P_{n}^{m} \cap T_{n}^{m} \subseteq P G_{n}^{m} \cap T G_{n}^{m}=I G_{n}^{m}$.

(b): $P_{n}^{m} \subseteq F_{n}^{m} \cap P G_{n}^{m}$ and $T_{n}^{m} \subseteq F_{n}^{m} \cap T G_{n}^{m}$ by (a). Thus take $A \in F_{n}^{m}$ and $f=f_{A}$ (see Remark 8). If $A \in T G_{n}^{m}$, then $A[n, 1]=n$, so $f \in T(n, m)$. Hence 
$A \in T_{n}^{m}$. Next, assume that $f \notin P(n, m)$, i.e., $f(i)=f(j) \geqslant 1$ for some $i<j$. If $f(j)<m$, then $A[j, f(j)]=M(f)[j, f(j)]=\sum_{k \leqslant j, l \geqslant f(j)} C(f)[k, l] \geqslant C(f)[i, f(i)]+$ $C(f)[j, f(j)]+\sum_{k \leqslant j, l \geqslant f(j)+1} C(f)[k, l]=2+A[j, f(j)+1]$. If $f(j)=m$, then $A[j, m]=$ $\sum_{k \leqslant j, l=m} C(f)[k, l] \geqslant C(f)[i, m]+C(f)[j, m]=2$. These cases imply $A \notin P G_{n}^{m}$, so $F_{n}^{m} \cap P G_{n}^{m} \subseteq P_{n}^{m}$.

Finally, $F_{n}^{m} \cap I G_{n}^{m}=F_{n}^{m} \cap P G_{n}^{m} \cap T G_{n}^{m}=F_{n}^{m} \cap P G_{n}^{m} \cap F_{n}^{m} \cap T G_{n}^{m}=P_{n}^{m} \cap T_{n}^{m}=I_{n}^{m}$.

Take a matrix $A \in F G_{n}^{1}$. Then the unique column of $O^{r}(A)=O(A)$ can be considered as a function $f$ from $\{1,2, \ldots, n\}$ to $\{0,1\}$. Of course, $M^{c}(f)=O^{r}(A)$, so $M(f)=$ $A$. Hence we obtain that $F G_{n}^{1}=F_{n}^{1}$ for all $n \in \mathbb{N}^{*}$. In particular, $\left(F_{n}^{1}, \leqslant_{M}\right)$ is a finite distributive lattice for each $n \in \mathbb{N}^{*}$. Observe also that $\left(F_{n}^{1}, \leqslant_{M}\right)$ is not linearly ordered for $n \geqslant 3$, because the supremum of $\left[\begin{array}{lllll}1 & 1 & 1 & \ldots & 1\end{array}\right]^{T}$ and $\left[\begin{array}{lllll}0 & 1 & 2 & \ldots & 2\end{array}\right]^{T}$ is equal to $\left[\begin{array}{lllll}1 & 1 & 2 & \ldots & 2\end{array}\right]^{T}$. Note that these matrices correspond to functions $(1,0,0,0, \ldots, 0)$, $(0,1,1,0, \ldots, 0)$ and $(1,0,1,0, \ldots, 0)$, respectively. Obviously, lattices $F_{1}^{1}=\{[0],[1]\}$ and $F_{2}^{1}=\left\{\left[\begin{array}{ll}0 & 0\end{array}\right]^{T},\left[\begin{array}{ll}0 & 1\end{array}\right]^{T},\left[\begin{array}{ll}1 & 1\end{array}\right]^{T},\left[\begin{array}{ll}1 & 2\end{array}\right]^{T},\right\}$ are chains.

Since $\left(F(n, 1), \leqslant_{F}\right)$ and $\left(F_{n}^{1}, \leqslant_{M}\right)$ are isomorphic, we have also that $F(n, 1)$ is a finite distributive lattice for all $n \in \mathbb{N}^{*}$. Next, $F(n, 1)$ is a chain iff $n=1,2$. Note that $F(1,1)=\{(0),(1)\}$ and $F(2,1)=\{(0,0),(0,1),(1,0),(1,1)\}$.

For all $n, m \geqslant 2$, posets $\left(F_{n}^{m}, \leqslant_{M}\right)$ and $\left(P_{n}^{m}, \leqslant_{M}\right)$, thus also posets $\left(F(n, m), \leqslant_{F}\right)$ and $\left(P(n, m), \leqslant_{F}\right)$, do not form lattices. Take four functions $f_{1}=(1,0,0, \ldots, 0), f_{2}=$ $(0,2,0, \ldots, 0), g_{1}=(1,2,0, \ldots, 0), g_{2}=(2,0,0, \ldots, 0) \in P(n, m) \subseteq F(n, m)$ (see Figure in Subsection 1.3) and let $A=M\left(f_{1}\right), B=M\left(f_{2}\right), C=M\left(g_{1}\right), D=M\left(g_{2}\right)$, i.e.,

$$
\begin{gathered}
A=\left[\begin{array}{ccccc}
1 & 0 & 0 & \cdots & 0 \\
1 & 0 & 0 & \cdots & 0 \\
1 & 0 & 0 & \cdots & 0 \\
\vdots & \vdots & \vdots & \cdots & \vdots \\
1 & 0 & 0 & \cdots & 0
\end{array}\right], \quad B=\left[\begin{array}{ccccc}
0 & 0 & 0 & \cdots & 0 \\
1 & 1 & 0 & \cdots & 0 \\
1 & 1 & 0 & \cdots & 0 \\
\vdots & \vdots & \vdots & \cdots & \vdots \\
1 & 1 & 0 & \cdots & 0
\end{array}\right], \\
C=\left[\begin{array}{ccccc}
1 & 0 & 0 & \cdots & 0 \\
2 & 1 & 0 & \cdots & 0 \\
2 & 1 & 0 & \cdots & 0 \\
\vdots & \vdots & \vdots & \cdots & \vdots \\
2 & 1 & 0 & \cdots & 0
\end{array}\right], \quad D=\left[\begin{array}{ccccc}
1 & 1 & 0 & \cdots & 0 \\
1 & 1 & 0 & \cdots & 0 \\
1 & 1 & 0 & \cdots & 0 \\
\vdots & \vdots & \vdots & \cdots & \vdots \\
1 & 1 & 0 & \cdots & 0
\end{array}\right] .
\end{gathered}
$$

Assume that $S \in F_{n}^{m}$ is a supremum of $A$ and $B$. Then $A, B \leqslant_{M} S$, so $S[1,1] \geqslant$ $\max \{A[1,1], B[1,1]\}=1, S[1,2] \geqslant 0, S[2,1], S[2,2] \geqslant 1$. Next, $C$ and $D$ are upper bound of $A$ and $B$, so $S \leqslant{ }_{M} C, D$. Hence $S[1,1] \leqslant \min \{C[1,1], D[1,1]\}=1, S[1,2] \leqslant 0$, $S[2,1], S[2,2] \leqslant 1$. Summarizing, $S[1,1]=S[2,1]=S[2,2]=1$ and $S[1,2]=0$. Thus $O^{r}(S)[1,1]=1, O^{r}(S)[1,2]=0, O^{r}(S)[2,1]=0, O^{r}(S)[2,2]=1$, so $S \notin F_{n}^{m}$. This contradiction shows that $\{A, B\}$ has no supremum in $F_{n}^{m}$, thus also in $P_{n}^{m}$.

Posets $\left(I_{n}^{m}, \leqslant_{M}\right)$ and $\left(I(n, m), \leqslant_{F}\right)$ (where $\left.n \leqslant m\right)$ are not lattices for $n \geqslant 2$ and $m \geqslant 3$. Take four functions $f_{1}=(2,1,3,4,5, \ldots, n), f_{2}=(1,3,2,4,5, \ldots, n), g_{1}=$ 
$(2,3,1,4,5, \ldots, n), g_{2}=(3,1,2,4,5, \ldots, n) \in I(n, m)$ (see Figure in Subsection 1.3) and let $A=M\left(f_{1}\right), B=M\left(f_{2}\right), C=M\left(g_{1}\right), D=M\left(g_{2}\right)$, i.e.,

$$
\begin{aligned}
& A=\left[\begin{array}{cccc}
1 & 1 & 0 & \cdots \\
2 & 1 & 0 & \cdots \\
3 & 2 & 1 & \cdots \\
\vdots & \vdots & \vdots & \cdots
\end{array}\right], \quad B=\left[\begin{array}{cccc}
1 & 0 & 0 & \cdots \\
2 & 1 & 1 & \cdots \\
3 & 2 & 1 & \ldots \\
\vdots & \vdots & \vdots & \ldots
\end{array}\right], \\
& C=\left[\begin{array}{cccc}
1 & 1 & 0 & \cdots \\
2 & 2 & 1 & \cdots \\
3 & 2 & 1 & \cdots \\
\vdots & \vdots & \vdots & \cdots
\end{array}\right], \quad D=\left[\begin{array}{cccc}
1 & 1 & 1 & \cdots \\
2 & 1 & 1 & \cdots \\
3 & 2 & 1 & \cdots \\
\vdots & \vdots & \vdots & \ldots
\end{array}\right]
\end{aligned}
$$

Assume that $S \in I_{n}^{m}$ is a supremum of $A$ and $B$. Then $A, B \leqslant_{M} S$, so $S[1,1] \geqslant$ $\max \{A[1,1], B[1,1]\}=1, S[1,2], S[2.2], S[2,3] \geqslant 1, S[1,3] \geqslant 0, S[2,1] \geqslant 2$. Next, $C$ and $D$ are upper bound of $A$ and $B$, so $S \leqslant{ }_{M} C, D$. Hence $S[1,1] \leqslant \min \{C[1,1], D[1,1]\}=1$, $S[1,2], S[2,2], S[2,3] \leqslant 1, S[1,3] \leqslant 0, S[2,1] \leqslant 2$. Summarizing, $S[1,1]=S[1,2]=$ $S[2,2]=S[2,3]=1, S[2,1]=2$ and $S[1,3]=0$. Thus $O^{r}(S)[1,1]=O^{r}(S)[1,2]=$ $O^{r}(S)[2,1]=O^{r}(S)[2,3]=1$ and $O^{r}(S)[1,3]=O^{r}(S)[2,2]=0$. Hence $S \notin F_{n}^{m}$, in particular, $S \notin I_{n}^{m}$.

Recall that $\left(I_{1}^{m}, \leqslant_{M}\right)$ is an $m$-element chain. Next, $I_{2}^{2}=S_{2}^{2}=\left\{\left[\begin{array}{ll}1 & 0 \\ 2 & 1\end{array}\right],\left[\begin{array}{ll}1 & 1 \\ 2 & 1\end{array}\right]\right\}$ is a two-element chain. Hence and by the above fact we obtain that $\left(I_{n}^{m}, \leqslant_{M}\right)$, thus also $\left(I(n, m), \leqslant_{F}\right)$, is a lattice iff $n=m=2$ or $n=1$, because $I_{n}^{m}=\emptyset$ if $m<n$.

The bottom element $\mathbf{0}_{n}^{m}=\left[\begin{array}{cccc}0 & 0 & \ldots & 0 \\ 0 & 0 & \ldots & 0 \\ \vdots & \vdots & \ldots & \vdots \\ 0 & 0 & \ldots & 0\end{array}\right]$ of the lattice $\left(M_{n}^{m}(\mathbb{N}), \leqslant_{M}\right)$ is also the bottom element of lattices $\left(F G_{n}^{m}, \leqslant_{M}\right),\left(P G_{n}^{m}, \leqslant_{M}\right)$ and of posets $\left(F_{n}^{m}, \leqslant_{M}\right),\left(P_{n}^{m}, \leqslant_{M}\right)$.

Since $\mathbf{1}_{n}$ is the bottom element of $\left(T(n, m), \leqslant_{F}\right)$ (see the end of Subsection 1.2), we have that the matrix $M\left(\mathbf{1}_{n}\right)=\left[\begin{array}{cccc}1 & 0 & \ldots & 0 \\ 2 & 0 & \ldots & 0 \\ \vdots & \vdots & \ldots & \vdots \\ n & 0 & \ldots & 0\end{array}\right]$ is the bottom element of $\left(T_{n}^{m}, \leqslant M\right)$, which will be sometimes denoted by $\mathbf{0}_{T_{n}^{m}}$. Of course, $\mathbf{0}_{T_{n}^{m}}$ is the bottom element of $\left(T G_{n}^{m}, \leqslant_{M}\right)$ too. It also follows from facts that $T G_{n}^{1}=T_{n}^{1}=\left\{\mathbf{0}_{T_{n}^{1}}\right\}$ and for $m \geqslant 2$, the function $A \longmapsto \downarrow A$ is an isomorphism from $T G_{n}^{m}$ to $F G_{n}^{m-1}$ and $\downarrow \mathbf{0}_{T_{n}^{m}}=\mathbf{0}_{n}^{m-1}$.

Take the top element $\mathbf{m}_{n}$ of $\left(F(n, m), \leqslant_{F}\right)$ and $\left(T(n, m), \leqslant_{F}\right)$ (see the end of Subsection 1.2). Then $M\left(\mathbf{m}_{n}\right)=\left[\begin{array}{cccc}1 & 1 & \ldots & 1 \\ 2 & 2 & \ldots & 2 \\ \vdots & \vdots & \ldots & \vdots \\ n & n & \ldots & n\end{array}\right]$ is the top element of $\left(F_{n}^{m}, \leqslant_{M}\right)$ and $\left(T_{n}^{m}, \leqslant_{M}\right)$. The matrix $M\left(\mathbf{m}_{n}\right)$ will be denoted by $\mathbf{1}_{F_{n}^{m}}$. It is easy to see that $\mathbf{1}_{F_{n}^{m}}$ is also the top element of $\left(F G_{n}^{m}, \leqslant M\right)$ and $\left(T G_{n}^{m}, \leqslant_{M}\right)$. Note that $O^{r}\left(\mathbf{1}_{F_{n}^{m}}\right)=M^{c}\left(\mathbf{m}_{n}\right)=\left[\begin{array}{cccc}1 & 1 & \ldots & 1 \\ 1 & 1 & \ldots & 1 \\ \vdots & \vdots & \ldots & \vdots \\ 1 & 1 & \ldots & 1\end{array}\right]$ 
and $O\left(\mathbf{1}_{F_{n}^{m}}\right)=C\left(\mathbf{m}_{n}\right)=\left[\begin{array}{ccccc}0 & 0 & \ldots & 0 & 1 \\ 0 & 0 & \cdots & 0 & 1 \\ \vdots & \vdots & \ldots & \vdots & \vdots \\ 0 & 0 & \ldots & 0 & 1\end{array}\right]$

Take the top element $\nabla_{(n, m)}$ of $\left(P(n, m), \leqslant_{F}\right)$ (see the end of Subsection 1.2), which is also the top element of $\left(I(n, m), \leqslant_{F}\right)$ if $n \leqslant m$. Then $M\left(\nabla_{(n, m)}\right)$ is the top element of $\left(P_{n}^{m}, \leqslant_{M}\right)$ and also of $\left(I_{n}^{m}, \leqslant_{M}\right)$ if $n \leqslant m$. The matrix $M\left(\nabla_{(n, m)}\right)$ will be denoted by $\nabla_{n}^{m}$. Next, it is easy to see that $\nabla_{n}^{m}=\left[\begin{array}{ccccccc}1 & 1 & 1 & \cdots & 1 & 1 & 1 \\ 2 & 2 & 2 & \cdots & 2 & 2 & 1 \\ 3 & 3 & 3 & \cdots & 3 & 2 & 1 \\ \vdots & \vdots & \vdots & \cdots & \vdots & \vdots & \vdots \\ m-1 & m-1 & m-2 & \cdots & 3 & 2 & 1 \\ m & m-1 & m-2 & \cdots & 3 & 2 & 1 \\ \vdots & \vdots & \vdots & \cdots & \vdots & \vdots & \vdots \\ m & m-1 & m-2 & \cdots & 3 & 2 & 1\end{array}\right]$ if $m \leqslant n$, and $\nabla_{n}^{m}=\left[\begin{array}{ccccccccc}1 & 1 & \cdots & 1 & 1 & 1 & \cdots & 1 & 1 \\ 2 & 2 & \cdots & 2 & 2 & 2 & \cdots & 2 & 1 \\ \vdots & \vdots & \cdots & \vdots & \vdots & \vdots & \cdots & \vdots & \vdots \\ n-1 & n-1 & \cdots & n-1 & n-1 & n-2 & \cdots & 2 & 1 \\ n & n & \cdots & n & n-1 & n-2 & \cdots & 2 & 1\end{array}\right]$ if $n \leqslant m$. Hence it easily follows that $\nabla_{n}^{m}$ is the top element of $\left(P G_{n}^{m}, \leqslant M\right)$ and also of $\left(I G_{n}^{m}, \leqslant_{M}\right)$ if $n \leqslant m$. Note that $O^{r}\left(\nabla_{n}^{m}\right)=M^{c}\left(\nabla_{(n, m)}\right)=\left[\begin{array}{cccccc}1 & 1 & \cdots & 1 & 1 & 1 \\ 1 & 1 & \cdots & 1 & 1 & 0 \\ 1 & 1 & \cdots & 1 & 0 & 0 \\ \vdots & \vdots & \cdots & \vdots & \vdots & \vdots \\ 1 & 0 & \cdots & 0 & 0 & 0 \\ \vdots & \vdots & \cdots & \vdots & \vdots & \vdots \\ 0 & 0 & \cdots & 0 & 0 & 0\end{array}\right]$ if $m \leqslant n$, and $O^{r}\left(\nabla_{n}^{m}\right)=M^{c}\left(\nabla_{(n, m)}\right)=$ $\left[\begin{array}{cccccccc}1 & 1 & \cdots & 1 & 1 & \cdots & 1 & 1 \\ 1 & 1 & \cdots & 1 & 1 & \cdots & 1 & 0 \\ \vdots & \vdots & \cdots & \vdots & \vdots & \cdots & \vdots & \vdots \\ 1 & 1 & \cdots & 1 & 1 & \cdots & 0 & 0 \\ 1 & 1 & \cdots & 1 & 0 & \cdots & 0 & 0\end{array}\right]$ if $n \leqslant m$. Next, $O\left(\nabla_{n}^{m}\right)=C\left(\nabla_{(n, m)}\right)=\left[\begin{array}{cccccc}0 & 0 & \cdots & 0 & 0 & 1 \\ 0 & 0 & \cdots & 0 & 1 & 0 \\ 0 & 0 & \cdots & 1 & 0 & 0 \\ \vdots & \vdots & \cdots & \vdots & \vdots & \vdots \\ 1 & 0 & \cdots & 0 & 0 & 0 \\ \vdots & \vdots & \cdots & \vdots & \vdots & \vdots \\ 0 & 0 & \cdots & 0 & 0 & 0\end{array}\right]$ if $m \leqslant n$, and $O\left(\nabla_{n}^{m}\right)=C\left(\nabla_{(n, m)}\right)=\left[\begin{array}{cccccccc}0 & 0 & \cdots & 0 & 0 & \cdots & 0 & 1 \\ 0 & 0 & \cdots & 0 & 0 & \cdots & 1 & 0 \\ \vdots & \vdots & \cdots & \vdots & \vdots & \cdots & \vdots & \vdots \\ 0 & 0 & \cdots & 0 & 1 & \cdots & 0 & 0 \\ 0 & 0 & \cdots & 1 & 0 & \cdots & 0 & 0\end{array}\right]$ if $n \leqslant m$.

Finally, assume $n \leqslant m$ and take the bottom element $\Delta_{(n, m)}$ of $\left(I(n, m), \leqslant_{F}\right)$ (see the end of Subsection 1.2). Then $M\left(\Delta_{(n, m)}\right)$ is the bottom element of $\left(I_{n}^{m}, \leqslant_{M}\right)$. The matrix $M\left(\Delta_{(n, m)}\right)$ will be denoted by $\Delta_{n}^{m}$. Next, $\Delta_{n}^{m}=\left[\begin{array}{ccccccccc}1 & 0 & 0 & \cdots & 0 & 0 & \cdots & 0 & 0 \\ 2 & 1 & 0 & \cdots & 0 & 0 & \cdots & 0 & 0 \\ \vdots & \vdots & \vdots & \cdots & \vdots & \vdots & \cdots & \vdots & \vdots \\ n-1 & n-2 & n-3 & \cdots & 1 & 0 & \cdots & 0 & 0 \\ n & n-1 & n-2 & \cdots & 2 & 1 & \cdots & 0 & 0\end{array}\right]$, so it is the bottom element of $\left(I G_{n}^{m}, \leqslant_{M}\right)$, too. Note that $O^{r}\left(\Delta_{n}^{m}\right)=M^{c}\left(\Delta_{(n, m)}\right)=$ $\left[\begin{array}{ccccccccc}1 & 0 & 0 & \cdots & 0 & 0 & \cdots & 0 & 0 \\ 1 & 1 & 0 & \cdots & 0 & 0 & \cdots & 0 & 0 \\ \vdots & \vdots & \vdots & \cdots & \vdots & \vdots & \cdots & \vdots & \vdots \\ 1 & 1 & 1 & \cdots & 1 & 0 & \cdots & 0 & 0 \\ 1 & 1 & 1 & \cdots & 1 & 1 & \cdots & 0 & 0\end{array}\right], O\left(\Delta_{n}^{m}\right)=C\left(\Delta_{(n, m)}\right)=\left[\begin{array}{cccccccccc}1 & 0 & 0 & \cdots & 0 & 0 & 0 & \cdots & 0 & 0 \\ 0 & 1 & 0 & \cdots & 0 & 0 & 0 & \cdots & 0 & 0 \\ \vdots & \vdots & \vdots & \cdots & \vdots & \vdots & \vdots & \cdots & \vdots & \vdots \\ 0 & 0 & 0 & \cdots & 0 & 1 & 0 & \cdots & 0 & 0 \\ 0 & 0 & 0 & \cdots & 0 & 0 & 1 & \cdots & 0 & 0\end{array}\right]$. 
In particular, for $n=m$ we obtain that matrices $\nabla_{n}^{n}=\left[\begin{array}{cccccc}1 & 1 & 1 & \cdots & 1 & 1 \\ 2 & 2 & 2 & \cdots & 2 & 1 \\ \vdots & \vdots & \vdots & \cdots & \vdots & \vdots \\ n-2 & n-2 & n-2 & \cdots & 2 & 1 \\ n-1 & n-1 & n-2 & \cdots & 2 & 1 \\ n & n-1 & n-2 & \cdots & 2 & 1\end{array}\right]$ and $\Delta_{n}^{n}=\left[\begin{array}{cccccc}1 & 0 & 0 & \cdots & 0 & 0 \\ 2 & 1 & 0 & \cdots & 0 & 0 \\ \vdots & \vdots & \vdots & \cdots & \vdots & \vdots \\ n-1 & n-2 & n-3 & \cdots & 1 & 0 \\ n & n-1 & n-2 & \cdots & 2 & 1\end{array}\right]$ are the top and the bottom element of the lattice $\left(S G_{n}^{n}, \leqslant_{M}\right)$, respectively (obviously, these matrices are also the top and the bottom element of $\left(S_{n}^{n}, \leqslant_{M}\right)$, respectively). Note that $O^{r}\left(\nabla_{n}^{n}\right)=M^{c}\left(\nabla_{(n)}\right)=\left[\begin{array}{cccccc}1 & 1 & 1 & \cdots & 1 & 1 \\ 1 & 1 & 1 & \cdots & 1 & 0 \\ \vdots & \vdots & \vdots & \cdots & \vdots & \vdots \\ 1 & 1 & 0 & \cdots & 0 & 0 \\ 1 & 0 & 0 & \cdots & 0 & 0\end{array}\right]$, $O\left(\nabla_{n}^{n}\right)=C\left(\nabla_{(n)}\right)=\left[\begin{array}{cccccc}0 & 0 & 0 & \cdots & 0 & 1 \\ 0 & 0 & 0 & \cdots & 1 & 0 \\ \vdots & \vdots & \vdots & \cdots & \vdots & \vdots \\ 0 & 1 & 0 & \cdots & 0 & 0 \\ 1 & 0 & 0 & \cdots & 0 & 0\end{array}\right], O^{r}\left(\Delta_{n}^{n}\right)=M^{c}\left(\Delta_{(n)}\right)=\left[\begin{array}{cccccc}1 & 0 & 0 & \cdots & 0 & 0 \\ 1 & 1 & 0 & \cdots & 0 & 0 \\ \vdots & \vdots & \vdots & \cdots & \vdots & \vdots \\ 1 & 1 & 1 & \cdots & 1 & 0 \\ 1 & 1 & 1 & \cdots & 1 & 1\end{array}\right]$, $O\left(\Delta_{n}^{n}\right)=C\left(\Delta_{(n)}\right)=I_{n}^{n}=\left[\begin{array}{cccccc}1 & 0 & 0 & \cdots & 0 & 0 \\ 0 & 1 & 0 & \cdots & 0 & 0 \\ \vdots & \vdots & \vdots & \cdots & \vdots & \vdots \\ 0 & 0 & 0 & \cdots & 1 & 0 \\ 0 & 0 & 0 & \cdots & 0 & 1\end{array}\right]$

\subsection{Self-duality of lattices $\left(F G_{n}^{m}, \leqslant_{M}\right),\left(T G_{n}^{m}, \leqslant_{M}\right)$ and $\left(I G_{n}^{m}, \leqslant_{M}\right)$}

We know that the function $f \longmapsto \mathbf{m}_{n}-f$ is an involutive order anti-automorphism of $F(n, m)$ (Proposition 2). Now we show that this function can be transported on the poset $F_{n}^{m}$, and next, extended to an involutive anti-automorphism of the lattice $F G_{n}^{m}$. In particular, $F G_{n}^{m}$ is a self-dual lattice. We start with the following definition.

Definition 13. For each $A \in M_{n}^{m}(\mathbb{Z})$, let $A^{d}=\left(\mathbf{1}_{F_{n}^{m}}-A\right)^{h t}=\mathbf{1}_{F_{n}^{m}}^{h t}-A^{h t}=\mathbf{1}_{F_{n}^{m}}-A^{h t}$, i.e., $A^{d}[i, j]=i-A[i, m-j+1]$.

Lemma 14. For each $A \in F G_{n}^{m}, A^{d} \in F G_{n}^{m}$ and $\left(A^{d}\right)^{d}=A$.

Proof. The first part is obtained by a standard verification. Next, $\left(A^{d}\right)^{d}=\left(\mathbf{1}_{F}-\left(\mathbf{1}_{F}-\right.\right.$ $\left.A)^{h t}\right)^{h t}=\mathbf{1}_{F}-\left(\left(\mathbf{1}_{F}-A\right)^{h t}\right)^{h t}=\mathbf{1}_{F}-\left(\mathbf{1}_{F}-A\right)=A$.

For $A \in F G_{n}^{m}$, the matrix $A^{d}$ will be sometimes called the dual of $A$ in the lattice $\left(F G_{n}^{m}, \leqslant_{M}\right)$, because the following result holds.

\section{Theorem 15.}

(a) The function $A \longmapsto A^{d}$ is an involutive anti-automorphism of the $\operatorname{poset}\left(F G_{n}^{m}, \leqslant_{M}\right)$. In particular, the finite distributive lattice $\left(F G_{n}^{m}, \leqslant_{M}\right)$ is self-dual.

(b) Posets $\left(\operatorname{Jir}\left(F G_{n}^{m}\right), \leqslant_{M}\right)$ and $\left(\operatorname{Mir}\left(F G_{n}^{m}\right), \leqslant_{M}\right)$ are isomorphic to $\left(\operatorname{Mir}\left(F G_{n}^{m}\right), \leqslant_{M}^{d}\right)$ and $\left(\operatorname{Jir}\left(F G_{n}^{m}\right), \leqslant_{M}^{d}\right)$, respectively. 
(c) For each matrix $B \in F G_{n}^{m}, B \in \operatorname{Jir}\left(F G_{n}^{m}\right)$ (respectively, $B \in \operatorname{Mir}\left(F G_{n}^{m}\right)$ ) iff $B^{d} \in \operatorname{Mir}\left(F G_{n}^{m}\right)$ (respectively, $\left.B^{d} \in \operatorname{Jir}\left(F G_{n}^{m}\right)\right)$.

Proof. (a): By Lemma 14 this function is a well-defined involution from $F G_{n}^{m}$ to $F G_{n}^{m}$. Next, for $A, B \in F G_{n}^{m}, A \leqslant_{M} B$ iff $A^{h t} \leqslant_{M} B^{h t}$ iff $-B^{h t} \leqslant_{M}-A^{h t}$ iff $\mathbf{1}_{F}-B^{h t} \leqslant_{M}$ $\mathbf{1}_{F}-A^{\text {ht }}$ iff $B^{d} \leqslant_{M} A^{d}$ iff $A^{d} \leqslant_{M}^{d} B^{d}$.

Points (b) and (c) are obtained from (a).

Lemma 16. For each $A \in F G_{n}^{m}, O^{r}\left(A^{d}\right)=\left(O^{r}(A)^{n e g}\right)^{h t}$.

Proof. $O^{r}\left(A^{d}\right)=O^{r}\left(\mathbf{1}_{F}-A^{h t}\right)=O^{r}\left(\mathbf{1}_{F}\right)-O^{r}\left(A^{h t}\right)=\mathbf{1}_{n}^{m}-O^{r}\left(A^{h t}\right)=\left(O^{r}(A)^{h t}\right)^{n e g}=$ $\left(O^{r}(A)^{n e g}\right)^{h t}$.

Lemma 17. Let $f \in F(n, m)$ and $A \in F G_{n}^{m}$. Then

(a) $M(f)^{d}=M\left(\mathbf{m}_{n}-f\right)$.

(b) $A \in F_{n}^{m}$ iff $A^{d} \in F_{n}^{m}$.

Proof. (a): Let $A=M(f)$. The $i$-th row of $O^{r}(A)$ is equal to $[\underbrace{1,1, \ldots, 1}_{f(i)}, 0,0, \ldots, 0]$. Thus the $i$-th row of $\left(O^{r}(A)^{n e g}\right)^{h t}=O^{r}\left(A^{d}\right)$ (see Lemma 16) is equal to $[\underbrace{1,1, \ldots, 1}_{m-f(i)}, 0,0, \ldots, 0]$. Hence $O^{r}\left(M\left(\mathbf{m}_{n}-f\right)\right)=C^{c}\left(\mathbf{m}_{n}-f\right)=O^{r}\left(A^{d}\right)$, so $M\left(\mathbf{m}_{n}-f\right)=A^{d}$.

(b): It is obtained by (a) and the equality $\left(A^{d}\right)^{d}=A$.

By the above lemma we obtain the following fact.

Proposition 18. Take the involutive anti-automorphism $\Phi: A \longmapsto A^{d}$ of $\left(F G_{n}^{m}, \leqslant_{M}\right)$ and the isomorphism $\Psi: f \longmapsto M(f)$ between $\left(F(n, m), \leqslant_{F}\right)$ and $\left(F_{n}^{m}, \leqslant_{M}\right)$. Then $\Phi_{\left\lceil F_{n}^{m}\right.}$ and $\Psi^{-1} \circ \Phi_{\left\lceil F_{n}^{m}\right.} \circ \Psi$ are involutive anti-automorphisms of $\left(F_{n}^{m}, \leqslant_{M}\right)$ and $\left(F(n, m), \leqslant_{F}\right)$, respectively. Moreover, $\left.\Psi^{-1} \circ \Phi\right|_{F_{n}^{m}} \circ \Psi(f)=\mathbf{m}_{n}-f$ for all $f \in F(n, m)$, i.e., it is the function defined in Proposition 2.

Recall that the function $A \longmapsto \downarrow A$ is an isomorphism between lattices $\left(T G_{n}^{m}, \leqslant_{M}\right)$ and $\left(F G_{n}^{m-1}, \leqslant_{M}\right)$ (for $m \geqslant 2$ ). Thus by Theorem 15 we have that the finite distributive lattice $\left(T G_{n}^{m}, \leqslant_{M}\right)$ is self-dual and posets $\left(\operatorname{Jir}\left(T G_{n}^{m}\right), \leqslant_{M}\right),\left(\operatorname{Mir}\left(T G_{n}^{m}\right), \leqslant_{M}\right)$ are isomorphic to posets $\left(\operatorname{Mir}\left(T G_{n}^{m}\right), \leqslant_{M}^{d}\right),\left(\operatorname{Jir}\left(T G_{n}^{m}\right), \leqslant_{M}^{d}\right)$, respectively. Note that for $m=1$ these facts are obvious, because $T G_{n}^{1}=\left\{\left[\begin{array}{llll}1 & 2 \ldots n\end{array}\right]^{T}\right\}$ and consequently, $\operatorname{Jir}\left(T G_{n}^{1}\right)=\operatorname{Mir}\left(T G_{n}^{1}\right)=\emptyset$. Moreover, we can transport the function $A \longmapsto A^{d}$ on $T G_{n}^{m}$ to obtain an involutive anti-automorphism of $\left(T G_{n}^{m}, \leqslant_{M}\right)$. We will also show that this new anti-automorphism preserves $I G_{n}^{m}$, so $\left(I G_{n}^{m}, \leqslant_{M}\right)$ is self-dual too. Therefore we first introduce the following definition. 
Definition 19. For each $A \in M_{n}^{m}(\mathbb{Z})$, where $m \geqslant 2$, let $A^{t d}=\uparrow\left((\downarrow A)^{d}\right)$.

Recall that for every $n \times k$-matrix $B, \downarrow B$ is the $n \times(k-1)$-matrix (for $k \geqslant 2$ ) obtained from $B$ by removing the first column, i.e., $\downarrow B[i, j]=B[i, j+1]$ for $1 \leqslant i \leqslant n$ and $1 \leqslant j \leqslant k-1$. Next, $\uparrow B$ is the $n \times(k+1)$-matrix obtained from $B$ by adding the first column $\left[\begin{array}{lllll}1 & 2 & 3 & \ldots\end{array}\right]^{T}$, i.e., $\uparrow B[i, 1]=i$ and $\uparrow B[i, j]=B[i, j-1]$ for $1 \leqslant i \leqslant n$ and $2 \leqslant j \leqslant k+1$.

If $m=1$, then we set $A^{t d}=\left[\begin{array}{lllll}1 & 2 & 3 & \ldots\end{array}\right]^{T}$ for all $A \in M_{n}^{1}(\mathbb{Z})$.

Observe that for all $1 \leqslant i \leqslant n$ and $1 \leqslant j \leqslant m$,

$$
A^{t d}[i, j]=\left\{\begin{array}{ll}
i & \text { if } j=1 \\
i-A[i, m-j+2] & \text { if } 2 \leqslant j \leqslant m
\end{array},\right.
$$

because $A^{t d}[i, j]=(\downarrow A)^{d}[i, j-1]=i-(\downarrow A)[i,(m-1)-(j-1)+1]=i-(\downarrow A)[i, m-j+1]=$ $i-A[i, m-j+2]$ for each $j \geqslant 2$.

It is easy to see that the following facts holds. First, $\downarrow \uparrow A=A$ for each $n \times m$-matrix $A$. Second, $\downarrow A \in F G_{n}^{m-1}$ (if $m \geqslant 2$ ) and $\uparrow A \in T G_{n}^{m+1} \subseteq F G_{n}^{m+1}$ for all $A \in F G_{n}^{m}$. Third, for every $A \in F G_{n}^{m}$ (where $m \geqslant 2$ ), $\uparrow(\downarrow A)=A$ iff $A \in T G_{n}^{m}$.

\section{Lemma 20.}

(a) For each $A \in F G_{n}^{m}, A^{t d} \in T G_{n}^{m}$. Moreover, if $A \in I G_{n}^{m}$, then $A^{t d} \in I G_{n}^{m}$.

(b) For each $A \in T G_{n}^{m},\left(A^{t d}\right)^{t d}=A$.

Proof. (a): It is obvious for $m=1$. Thus assume $m \geqslant 2$. Then $\downarrow A \in F G_{n}^{m-1}$, so $(\downarrow A)^{d} \in F G_{n}^{m-1}$ by Lemma 14 . Hence $A^{t d}=\uparrow\left((\downarrow A)^{d}\right) \in T G_{n}^{m}$.

Assume that $A \in I G_{n}^{m}$. Since $A^{t d} \in T G_{n}^{m}$, it is sufficient to show that every two adjacent numbers on a row are equal or differ by 1 . Of course, all rows of $\downarrow A$ satisfy this condition, so it holds also for all rows of $(\downarrow A)^{h t}$. Consequently, each row of $(\downarrow A)^{d}=$ $\mathbf{1}_{F_{n}^{m-1}}-(\downarrow A)^{h t}$ satisfies this condition. Moreover, $A^{t d}[i, 2]=i-A[i, m]$ for $1 \leqslant i \leqslant n$. Thus $\left.A^{t d}[i, 1]-A^{t d}[i, 2]=i-(i-A[i, m])=A[i, m]\right)=0$ or 1 .

(b): $\left(A^{t d}\right)^{t d}=\left(\uparrow\left((\downarrow A)^{d}\right)\right)^{t d}=\uparrow\left(\left(\downarrow \uparrow\left((\downarrow A)^{d}\right)\right)^{d}\right)=\uparrow\left(\left((\downarrow A)^{d}\right)^{d}\right)=\uparrow(\downarrow A)=A$, the last equality holds, because $A \in T G_{n}^{m}$.

For $A \in T G_{n}^{m}$, the matrix $A^{t d}$ will be sometimes called the $t$-dual of $A$ in the lattice $\left(T G_{n}^{m}, \leqslant_{M}\right)$, because by Theorem 15 we have the following fact.

\section{Corollary 21.}

(a) The function $A \longmapsto A^{\text {td }}$ is an involutive anti-automorphism of $\left(T G_{n}^{m}, \leqslant_{M}\right)$. In particular, the finite distributive lattice $\left(T G_{n}^{m}, \leqslant_{M}\right)$ is self-dual.

(b) Posets $\left(\operatorname{Jir}\left(T G_{n}^{m}\right), \leqslant_{M}\right)$ and $\left(\operatorname{Mir}\left(T G_{n}^{m}\right), \leqslant_{M}\right)$ are isomorphic to $\left(\operatorname{Mir}\left(T G_{n}^{m}\right), \leqslant_{M}^{d}\right)$ and $\left(\operatorname{Jir}\left(T G_{n}^{m}\right), \leqslant_{M}^{d}\right)$, respectively. 
(c) For each matrix $A \in T G_{n}^{m}, A \in \operatorname{Jir}\left(T G_{n}^{m}\right)$ (respectively, $A \in \operatorname{Mir}\left(T G_{n}^{m}\right)$ ) iff $A^{t d} \in \operatorname{Mir}\left(T G_{n}^{m}\right)$ (respectively, $\left.A^{t d} \in \operatorname{Jir}\left(T G_{n}^{m}\right)\right)$.

Proof. Assume first that $m \geqslant 2$ and take the involutive anti-automorphism $\Theta: A \longmapsto$ $A^{d}$ of the lattice $\left(F G_{n}^{m-1}, \leqslant_{M}\right)$, the isomorphism $\Phi: A \longmapsto \downarrow A$ from $\left(T G_{n}^{m}, \leqslant_{M}\right)$ to $\left(F G_{n}^{m-1}, \leqslant_{M}\right)$ and its inverse $\Phi^{-1}: A \longmapsto \uparrow A$. Then the composition $\Phi^{-1} \circ \Theta \circ \Phi$ is an involutive anti-isomorphism of $\left(T G_{n}^{m}, \leqslant M\right)$ and $\Phi^{-1} \circ \Theta \circ \Phi(A)=A^{t d}$ for all $A \in T G_{n}^{m}$.

$A \longmapsto A^{t d}$ is also an involutive anti-automorphism of $\left(T G_{n}^{1}, \leqslant_{M}\right)$, because $T G_{n}^{1}=$ $\left.\left\{\begin{array}{lllll}1 & 2 & 3 & \ldots & n\end{array}\right]^{T}\right\}$.

Since $A^{t d} \in I G_{n}^{m}$ for all $A \in I G_{n}^{m}$ (Lemma 20), by Corollary 21 we obtain the following result.

\section{Corollary 22.}

(a) The function $A \longmapsto A^{\text {td }}$ is an involutive anti-automorphism of $\left(I G_{n}^{m}, \leqslant_{M}\right)$ (where $n \leqslant m)$. In particular, the finite distributive lattice $\left(I G_{n}^{m}, \leqslant_{M}\right)$ is self-dual.

(b) Posets $\left(\operatorname{Jir}\left(I G_{n}^{m}\right), \leqslant_{M}\right)$ and $\left(\operatorname{Mir}\left(I G_{n}^{m}\right), \leqslant_{M}\right)$ are isomorphic to $\left(\operatorname{Mir}\left(I G_{n}^{m}\right), \leqslant_{M}^{d}\right)$ and $\left(\operatorname{Jir}\left(I G_{n}^{m}\right), \leqslant_{M}^{d}\right)$, respectively.

(c) For each matrix $B \in I G_{n}^{m}, B \in \operatorname{Jir}\left(I G_{n}^{m}\right)$ (respectively, $\left.B \in \operatorname{Mir}\left(I G_{n}^{m}\right)\right)$ iff $B^{t d} \in$ $\operatorname{Mir}\left(I G_{n}^{m}\right)$ (respectively, $B^{t d} \in \operatorname{Jir}\left(I G_{n}^{m}\right)$ ).

We use the following notation in the next results. For each $n \times m$-matrix $B$ and $b_{1}, b_{2}, b_{3}, \ldots, b_{n} \in \mathbb{Z}, \uparrow_{b_{1}, b_{2}, b_{3}, \ldots, b_{n}} B$ denotes the $n \times(m+1)$-matrix obtained from $B$ by adding the first column $\left[\begin{array}{lllll}b_{1} & b_{2} & b_{3} & \ldots & b_{n}\end{array}\right]^{T}$.

Lemma 23. For each $A \in T G_{n}^{m}, O^{r}\left(A^{t d}\right)=\uparrow_{1,1, \ldots, 1}\left(\left(O^{r}(\downarrow A)^{n e g}\right)^{h t}\right)$.

Proof. $O^{r}\left(A^{t d}\right)=O^{r}\left(\uparrow\left((\downarrow A)^{d}\right)\right)=\uparrow_{1,1, \ldots, 1} O^{r}\left((\downarrow A)^{d}\right)=\left(\right.$ Lemma 16) $=_{1,1, \ldots, 1}\left(\left(O^{r}(\downarrow\right.\right.$ $\left.\left.A)^{n e g}\right)^{h t}\right)$.

Lemma 24. Let $f \in T(n, m)$ and $A \in T G_{n}^{m}$. Then

(a) $M(f)^{t d}=M\left(\mathbf{m}_{n}-f+\mathbf{1}_{n}\right)$.

(b) $A \in T_{n}^{m}$ (respectively, $\left.A \in I_{n}^{m}\right)$ iff $A^{t d} \in T_{n}^{m}$ (respectively, $A^{t d} \in I_{n}^{m}$ ).

Proof. (a): Let $A=M(f)$. The $i$-th row of the matrix $O^{r}(\downarrow A)=\downarrow O^{r}(A)$ is equal to $\overbrace{[\underbrace{1,1, \ldots, 1}_{f(i)-1}, 0,0, \ldots, 0]}^{m-1}$. Thus the $i$-th row of $\left(O^{r}(\downarrow A)^{n e g}\right)^{h t}$ equals $\overbrace{[\underbrace{1,1, \ldots, 1}_{m-f(i)}, 0,0, \ldots, 0]}^{m-1}$.

Next, by Lemma 23 we have the equality $O^{r}\left(A^{t d}\right)=\uparrow_{1,1, \ldots, 1}\left(\left(O^{r}(\downarrow A)^{n e g}\right)^{h t}\right)$. These two facts imply that the $i$-th row of the matrix $O^{r}\left(A^{t d}\right)$ equals $\overbrace{\underbrace{1,1, \ldots, 1}_{m-f(i)+1}, 0,0, \ldots, 0]}^{m}$. Hence $O^{r}\left(M\left(\mathbf{m}_{n}-f+\mathbf{1}_{n}\right)\right)=C^{c}\left(\mathbf{m}_{n}-f+\mathbf{1}_{n}\right)=O^{r}\left(A^{t d}\right)$, so $M\left(\mathbf{m}_{n}-f+\mathbf{1}_{n}\right)=A^{t d}$.

(b): It is obtained by (a) and the equality $\left(A^{t d}\right)^{t d}=A$ which holds for all $A \in T G_{n}^{m}$. 
By the above lemma we obtain the following fact.

Proposition 25. Take the involutive anti-automorphism $\Phi: A \longmapsto A^{\text {td }}$ of $\left(T G_{n}^{m}, \leqslant_{M}\right)$ and the isomorphism $\Psi: f \longmapsto M(f)$ between $\left(T(n, m), \leqslant_{F}\right)$ and $\left(T_{n}^{m}, \leqslant_{M}\right)$. Then

(a) $\Phi_{\left\lceil T_{n}^{m}\right.}$ and $\Psi^{-1} \circ \Phi_{\left\lceil T_{n}^{m}\right.} \circ \Psi$ are involutive anti-automorphisms of posets $\left(T_{n}^{m}, \leqslant_{M}\right)$ and $\left(T(n, m), \leqslant_{F}\right)$, respectively. Moreover, $\left(\Psi^{-1} \circ \Phi_{\mid T_{n}^{m}} \circ \Psi\right)(f)=\mathbf{m}_{n}-f+\mathbf{1}_{n}$ for all $f \in T(n, m)$, i.e., it is the function defined in Corollary 3.

(b) If $n \leqslant m$, then $\Phi_{\left\lceil I_{n}^{m}\right.}$ and $\left(\Psi^{-1} \circ \Phi_{\left\lceil T_{n}^{m}\right.} \circ \Psi\right)_{\lceil I(n, m)}=\left(\Psi_{\lceil I(n, m)}\right)^{-1} \circ \Phi_{\left\lceil I_{n}^{m}\right.} \circ \Psi_{\lceil I(n, m)}$ are involutive anti-automorphisms of $\left(I_{n}^{m}, \leqslant_{M}\right)$ and $\left(I(n, m), \leqslant_{F}\right)$, respectively.

\subsection{Row-alternating matrices, alternating matrices and row-alternating sign matrices}

Definition 26. (a) We say that $A \in M_{n}^{m}(\{-1,0,1\})$ is a row-alternating matrix if the following five conditions hold:

(ra.1) the sum of all entries on each row is 0 or 1 ;

(ra.2) for each $1 \leqslant k \leqslant n$, the sum of first $k$ entries on each column is non-negative;

(ra.3) numbers 1 and -1 alternate on each row;

(ra.4) the first non-zero entry (if exists) on each column is 1 ;

(ra.5) the last non-zero entry (if exists) on each row is 1.

The set of all quasi-alternating matrices of size $n \times m$ will be denoted by $\mathcal{R} \mathcal{A}_{n}^{m}$.

Note that (ra.1) follows (ra.3) and (ra.5), and (ra.4) from (ra.2). Next, (ra.3) and (ra.4) imply that the first row of row-alternating matrix contains at most one 1.

(b) $A \in M_{n}^{m}(\{-1,0,1\})$ is called an alternating matrix if the following six conditions hold:

(al.1) the sum of all entries on each row is 0 or 1 ;

(al.2) the sum of all entries on each column is 0 or 1 ;

(al.3) numbers 1 and -1 alternate on each row;

(al.4) numbers 1 and -1 alternate on each column;

(al.5) the first non-zero entry (if exists) on each column is 1 ;

(al.6) the last non-zero entry (if exists) on each row is 1.

The set of all alternating matrices of size $n \times m$ will be denoted by $\mathcal{A}_{n}^{m}$.

Note that (al.1) follows from (al.3) and (al.6), and (al.2) from (al.4) and (al.5). Next, (al.4) and (al.6) imply that the last column of alternating matrix contains at most one 1. Similarly, by (al.3) and (al.5) we have that the first row of alternating matrix contains at most one 1 . 
(c) An alternating matrix is called a row-alternating sign matrix if the sum of all entries on each row is 1 . The set of all row-alternating sign matrices of size $n \times m$ will be denoted by $\mathcal{R} \mathcal{A} \mathcal{S}_{n}^{m}$.

The concept of alternating matrices has been introduced in [7] for square matrices. Take $A \in \mathcal{A}_{n}^{m}$. It follows from (al.4) and (al.5) that the sum of first $k$ entries on each column of $A$ is 0 or 1 , so (ra.2) holds for $A$. Hence $\mathcal{A}_{n}^{m} \subseteq \mathcal{R} \mathcal{A}_{n}^{m}$. Of course, we have also $\mathcal{R}_{\mathcal{A}} \mathcal{S}_{n}^{m} \subseteq \mathcal{A}_{n}^{m}$. Next, we easily obtain that $A \in \mathcal{A}_{n}^{m}$ iff $A \in \mathcal{R} \mathcal{A}_{n}^{m}$ and $A^{q t} \in \mathcal{R} \mathcal{A}_{m}^{n}$.

Recall that a square alternating matrix is called an alternating sign matrix (see [2]) if the sum of all entries on each row and on each column is 1 . The set of all alternating sign matrices of size $n \times n$ will be denoted by $\mathcal{A S}_{n}^{n}$. Of course, $\mathcal{A} \mathcal{S}_{n}^{n} \subseteq \mathcal{R} \mathcal{A} \mathcal{S}_{n}^{n}$. It is also obvious that $A \in \mathcal{A S}_{n}^{n}$ iff $A \in \mathcal{R} \mathcal{A S}_{n}^{n}$ and $A^{q t} \in \mathcal{R} \mathcal{A S}_{n}^{n}$.

It is proved in [7] that $P G_{n}^{n}$ and $\mathcal{A}_{n}^{n}$ have the same number of elements. Now we show analogous results for $F G_{n}^{m}, P G_{n}^{m}, T G_{n}^{m}, I G_{n}^{m}$. These results may be proved using techniques from [7], but we formulate other proofs which apply matrix operators $O^{r}$ and $O$. We first prove two technical facts.

Lemma 27. For each $A \in M_{n}^{m}(\mathbb{N})$,

(a) $A \in F G_{n}^{m}$ iff $O(A) \in \mathcal{R} \mathcal{A}_{n}^{m}$.

(b) $A \in P G_{n}^{m}$ iff $O(A) \in \mathcal{A}_{n}^{m}$.

(c) $A \in I G_{n}^{m}$ iff $O(A) \in \mathcal{R} \mathcal{A} \mathcal{S}_{n}^{m}$.

(d) $A \in S G_{n}^{n}$ iff $O(A) \in \mathcal{A S}_{n}^{n}$.

Proof. (a): Assume that $A \in F G_{n}^{m}$. Then $O^{r}(A) \in M_{n}^{m}(1)$, so $O(A) \in M_{n}^{m}(\{-1,0,1\})$ and $O(A)$ satisfies (ra.5). Next, we have that $\sum_{k \leqslant i, l \geqslant j} O(A)[k, l]=A[i, j] \geqslant A[i, j+1]=$ $\sum_{k \leqslant i, l \geqslant j+1} O(A)[k, l]$, so $\sum_{k=1}^{i} O(A)[k, j] \geqslant 0$, i.e., (ra.2) holds for $A$.

Take the $i$-th row $R$ of the matrix $O^{r}(A)$. If $R$ is non-zero, then $R$ is of the form $[\ldots, 0,1, \ldots, 1,0 \ldots, 0,1, \ldots, 1,0, \ldots]$. Each fragment $0,1, \ldots, 1,0$ of $R$ is transformed to $-1,0, \ldots, 0,1,0$ in the $i$-th row $R^{\prime}$ of $O(A)$. Of course, if $R=[1, \ldots, 1,1,0, \ldots]$, then $R^{\prime}=[0, \ldots, 0,1,0, \ldots]$. Thus $O(A)$ satisfies (ra.3).

To see (ra.4) assume that the first non-zero entry of a $j$-th column of $O(A)$ is -1 in the position $i$. Then $j \leqslant m-1$, because $A$ satisfies (ra.5). Next, by the above results concerning rows of $O^{r}(A)$ and $O(A)$ we obtain that $O^{r}(A)[i, j]=0, O^{r}(A)[i, j+1]=1$ and $O^{r}(A)[k, j]=O^{r}(A)[k, j+1]=0$ or $O^{r}(A)[k, j]=O^{r}(A)[k, j+1]=1$ for each $k<i$. These facts imply $A[i, j]=\sum_{k=1}^{i} O^{r}(A)[k, j]<\sum_{k=1}^{i} O^{r}(A)[k, j+1]=A[i, j+1]$. This contradiction shows that $O(A)$ satisfies (ra.4).

Now assume that $O(A) \in \mathcal{R} \mathcal{A}_{n}^{m}$. Then we have that $A[i, j]=\sum_{k \leqslant i, l \geqslant j} O(A)[k, l]=$ $\sum_{k \leqslant i, l \geqslant j+1} O(A)[k, l]+\sum_{k=1}^{i} O(A)[k, j] \geqslant \sum_{k \leqslant i, l \geqslant j+1} O(A)[k, l]=A[i, j+1]$ by (ra.2), so each row of $A$ is decreasing. Next, (ra.3) and (ra.5) imply $\sum_{l=j}^{m} O(A)[i, l]=0$ or 1 . Thus $A[i, j]-A[i-1, j]=\sum_{k \leqslant i, l \geqslant j} O(A)[k, l]-\sum_{k \leqslant i-1, l \geqslant j} O(A)[k, l]=\sum_{l=j}^{m} O(A)[i, l]=0$ or 1 , where we set $A[0, j]=O(A)[0, j]=0$. In particular, $A[1, j]=0$ or 1 . 
(b): We know that $A \in P G_{n}^{m}$ iff $A \in F G_{n}^{m}$ and $A^{q t} \in F G_{m}^{n}$. Hence $A \in P G_{n}^{m}$ iff $O(A) \in \mathcal{R} \mathcal{A}_{n}^{m}$ and $O(A)^{q t}=O\left(A^{q t}\right) \in \mathcal{R} \mathcal{A}_{m}^{n}$ iff $O(A) \in \mathcal{A}_{n}^{m}$.

(c): Assume that $A \in I G_{n}^{m}$. Then $O(A) \in \mathcal{A}_{n}^{m}$ by (b). Since the first column of $A$ is $[1,2, \ldots, n]^{T}$, each row of $O^{r}(A)$ is of the form $[1,1, \ldots, 1,0, \ldots]$. Hence the first non-zero entry on each row of $O(A)$ is 1 . Thus (al.3) and (al.6) imply that the sum of all entries on each row of $O(A)$ is 1 . So $O(A) \in \mathcal{R} \mathcal{A S}_{n}^{m}$.

Now assume that $O(A) \in \mathcal{R A S}_{n}^{m}$. Then $A \in P G_{n}^{m}$ by (b). Next, we have that $A[i, 1]=\sum_{k \leqslant i, l \geqslant 1} O(A)[k, l]=\sum_{k=1}^{i}\left(\sum_{l=1}^{m} O(A)[k, l]\right)=\sum_{k=1}^{i} 1=i$. Thus $A \in I G_{n}^{m}$.

(d): " $\Longleftarrow "$ follows from (c) (for $n=m$ ), because $\mathcal{A S}_{n}^{n} \subseteq \mathcal{R} \mathcal{A S}_{n}^{n}$. Assume that $A \in S G_{n}^{n}$. Then we know that $A^{q t} \in S G_{n}^{n}$. Hence and by (c), $O(A)$ and $O(A)^{q t}=O\left(A^{q t}\right)$ belong to $\mathcal{R} \mathcal{A S}_{n}^{n}$. Thus $O(A) \in \mathcal{A S}_{n}^{n}$.

Lemma 28. Let $A, B \in M_{n}^{m}(\mathbb{Z})$ be matrices such that $A[i, j]=\sum_{k \leqslant i, l \geqslant j} B[k, l]$. Then $O(A)=B$.

Proof. $O^{r}(A)=A[i, j]-A[i-1, j]=\sum_{l \geqslant j} B[i, l]$, so $O(A)=O^{r}(A)[i, j]-O^{r}(A)[i, j+1]=$ $B[i, j]$.

Since the function $A \longmapsto O(A)$ is injective, by Lemmas 27 and 28 we obtain the following result (recall that $T G_{n}^{m+1}$ is isomorphic to $F G_{n}^{m}$ ).

Theorem 29. $\left|F G_{n}^{m}\right|=\left|\mathcal{R} \mathcal{A}_{n}^{m}\right|=\left|T G_{n}^{m+1}\right|,\left|P G_{n}^{m}\right|=\left|\mathcal{A}_{n}^{m}\right|,\left|I G_{n}^{m}\right|=\left|\mathcal{R} \mathcal{A} \mathcal{S}_{n}^{m}\right|$ and $\left|S G_{n}^{n}\right|=\left|\mathcal{A} \mathcal{S}_{n}^{n}\right|$.

Since $\mathcal{A S}_{n}^{n} \subseteq \mathcal{R} \mathcal{A} \mathcal{S}_{n}^{n}$ and $\left|\mathcal{A S}_{n}^{n}\right|=\left|S G_{n}^{n}\right|=\left|\mathcal{R} \mathcal{A} \mathcal{S}_{n}^{n}\right|$, we obtain the following fact.

Corollary 30. $\mathcal{A S}_{n}^{n}=\mathcal{R} \mathcal{A S}_{n}^{n}$.

If $n \neq m$, then the sum of all entries on a column of a row-alternating sign matrix need not be 1 , in general. For example, take the matrix $\left[\begin{array}{cccc}0 & 0 & 1 & 0 \\ 0 & 1 & -1 & 1 \\ 1 & -1 & 1 & 0\end{array}\right]$ which obviously belongs to $\in \mathcal{R} \mathcal{A S}_{3}^{4}$.

Next, since $I G_{n}^{m}=\emptyset$ for $n>m$, by Theorem 29 we obtain

Remark 31. $\mathcal{R A S}_{n}^{m}=\emptyset$ for $n>m$.

By the above results we obtain the following fact that describes, using only the matrix operator $O$ whose matrices of $F G_{n}^{m}, P G_{n}^{m}$ and $I G_{n}^{m}$, respectively, belong to $F_{n}^{m}, P_{n}^{m}$ and $I_{n}^{m}$.

Proposition 32. Let $A \in M_{n}^{m}\left(\mathbb{N}^{*}\right)$. Then

(a) $A \in F_{n}^{m}$ iff $A \in F G_{n}^{m}$ and $O(A) \in M_{n}^{m}(1)$ iff $O(A) \in \mathcal{R} \mathcal{A}_{n}^{m} \cap M_{n}^{m}(1)$.

(b) $A \in P_{n}^{m}$ iff $A \in P G_{n}^{m}$ and $O(A) \in M_{n}^{m}(1)$ iff $O(A) \in \mathcal{A}_{n}^{m} \cap M_{n}^{m}(1)$.

(c) $A \in I_{n}^{m}$ iff $A \in I G_{n}^{m}$ and $O(A) \in M_{n}^{m}(1)$ iff $O(A) \in \mathcal{R}_{\mathcal{A}} \mathcal{S}_{n}^{m} \cap M_{n}^{m}(1)$.

(d) $A \in S_{n}^{n}$ iff $A \in S G_{n}^{n}$ and $O(A) \in M_{n}^{m}(1)$ iff $O(A) \in \mathcal{A S}_{n}^{n} \cap M_{n}^{m}(1)$. 
Proof. Observe first that (d) is obtained by (c) and Corollary 30.

Take $A \in F_{n}^{m}$ and its corresponding function $f_{A} \in F(n, m)$ (see Remark 8). Then $O(A)=C\left(f_{A}\right) \in M_{n}^{m}(1)$, because $A=M\left(f_{A}\right)$. Recall also that $F_{n}^{m} \subseteq F G_{n}^{m}, P_{n}^{m} \subseteq$ $P G_{n}^{m}$ and $I_{n}^{m} \subseteq I G_{n}^{m}$ (Proposition 12(a)). Hence we obtain implications " $\Longrightarrow$ "of first equivalences in (a), (b) and (c). Second equivalences in these three points follow from Lemma 27.

If $O(A) \in \mathcal{R} \mathcal{A}_{n}^{m} \cap M_{n}^{m}(1)$, then $O(A)$ has at most one entry 1 in each row by (ra.3). Let $f \in F(n, m)$ be a function such that $f(i)=0$ if the $i$-th row of $O(A)$ contains only zeros, and $O(A)[i, f(i)]=1$ otherwise. Then $O(A)=C(f)=O(M(f))$, so $A=M(f) \in F_{n}^{m}$. If additionally $O(A) \in \mathcal{A}_{n}^{m}$ (respectively, $O(A) \in \mathcal{R} \mathcal{A S}_{n}^{m}$ ), then $A \in F_{n}^{m} \cap P G_{n}^{m}=P_{n}^{m}$ (respectively, $A \in F_{n}^{m} \cap I G_{n}^{m}=I_{n}^{m}$ ) by Proposition 12(b) and Lemma 27.

Applying isomorphisms $A \longmapsto \downarrow A$ and $A \longmapsto \uparrow A$ between lattices $\left(T G_{n}^{m}, \leqslant_{M}\right)$ and $\left(F G_{n}^{m-1}, \leqslant_{M}\right.$ ) (for $m \geqslant 2$ ), all results of this subsection that concern $F G_{n}^{m}$ can be translated for $T G_{n}^{m}$ (where $m \geqslant 2$ ). For example, by Lemma 27(a) we have that for each $A \in M_{n}^{m}\left(\mathbb{N}^{*}\right)$ (and $\left.m \geqslant 2\right), A \in T G_{n}^{m}$ iff $\downarrow O(A)=O(\downarrow A) \in \mathcal{R} \mathcal{A}_{n}^{m-1}$ and the first column of $A$ equals $\left[\begin{array}{lllll}1 & 2 & 3 & \ldots & n\end{array}\right]^{T}$.

Now we show that $T G_{n}^{m}$ can be characterized in a similar way as $F G_{n}^{m}$. Let $\mathcal{B}_{n}^{m}$ be a set of all matrices $A \in \mathcal{R} \mathcal{A}_{n}^{m}$ such that the sum of all entries on each row of $A$ is 1 . Then

(T.1) For each $A \in M_{n}^{m}\left(\mathbb{N}^{*}\right), A \in T G_{n}^{m}$ iff $O(A) \in \mathcal{B}_{n}^{m}$.

$" \Longrightarrow "$ : By Lemma 27(a) we have that $O(A) \in \mathcal{R} \mathcal{A}_{n}^{m}$, because $T G_{n}^{m} \subseteq F G_{n}^{m}$. Next, in the same way as in the proof of the implication " $\Longrightarrow$ " of (c) of Lemma 27 we obtain that the sum of all entries on each row of $A$ is 1 .

$" \Longleftarrow "$ : By Lemma 27(a) we have that $A \in F G_{n}^{m}$, because $\mathcal{B}_{n}^{m} \subseteq \mathcal{R} \mathcal{A}_{n}^{m}$. Next, in the same way as in the proof of the implication " $\Longleftarrow$ of (c) of Lemma 27 we obtain that the first column of $A$ equals $\left[\begin{array}{lllll}1 & 2 & 3 & \ldots & n\end{array}\right]^{T}$. Thus $A \in T G_{n}^{m}$.

Since the function $A \longmapsto O(A)$ is injective, by Lemma 28 and (T.1) we obtain the following equality.

$$
\left|T G_{n}^{m}\right|=\left|\mathcal{B}_{n}^{m}\right|
$$

Hence and by Theorem 29 we have, in particular, the following interesting fact.

$$
\left|\mathcal{B}_{n}^{m+1}\right|=\left|\mathcal{R} \mathcal{A}_{n}^{m}\right| \cdot
$$

Proposition 32 takes the following form for $T_{n}^{m}$.

(T.3) $A \in T_{n}^{m}$ iff $A \in T G_{n}^{m}$ and $O(A) \in M_{n}^{m}(1)$ iff $O(A) \in \mathcal{B}_{n}^{m} \cap M_{n}^{m}(1)$.

The implication " $\Longrightarrow$ " of the first equivalence is obtained in the same way as in the proof of Proposition 32. The second equivalence follows from (T.1). Next, if $O(A) \in \mathcal{B}_{n}^{m} \cap$ $M_{n}^{m}(1)$, then $O(A) \in \mathcal{R} \mathcal{A}_{n}^{m} \cap M_{n}^{m}(1)$ and $A \in T G_{n}^{m}$ (by (T.1)), so $A \in F_{n}^{m} \cap T G_{n}^{m}=T_{n}^{m}$ by Propositions 12(b) and 32(a). 


\section{Dedekind-MacNeille completions}

It is shown in [7] that the lattice $\left(P G_{n}^{n}, \leqslant_{M}\right)$ is (up to isomorphism) the DedekindMacNeille completion of the poset $\left(P_{n}^{n}, \leqslant_{M}\right)$, so also of $\left(P(n), \leqslant_{F}\right)$. To this purpose (see Theorem 35 in the subsection below) M. Fortin has described join- and meet-irreducible elements of $\left(P G_{n}^{n}, \leqslant_{M}\right)$ and has shown that they belong to $P_{n}^{n}$. Now we use Fortin's methods to describe join- and meet-irreducible elements for every finite sublattice of the lattice $\left(M_{n}^{m}(\mathbb{Z}), \leqslant_{M}\right)$. In particular, join- and meet-irreducible elements of lattices $\left(F G_{n}^{m}, \leqslant_{M}\right),\left(P G_{n}^{m}, \leqslant_{M}\right),\left(T G_{n}^{m}, \leqslant_{M}\right)$ and $\left(I G_{n}^{m}, \leqslant_{M}\right)$ are found. We also show that these elements belong to $F_{n}^{m}, P_{n}^{m}, T_{n}^{m}$ and $I_{n}^{m}$, respectively. Hence we obtain that lattices $\left(F G_{n}^{m}, \leqslant_{M}\right),\left(P G_{n}^{m}, \leqslant_{M}\right),\left(T G_{n}^{m}, \leqslant_{M}\right)$ and $\left(I G_{n}^{m}, \leqslant_{M}\right)$ are Dedekind-MacNeille completions of posets $\left(F_{n}^{m}, \leqslant_{M}\right),\left(P_{n}^{m}, \leqslant_{M}\right),\left(T_{n}^{m}, \leqslant_{M}\right)$ and $\left(I_{n}^{m}, \leqslant_{M}\right)$, respectively, so also of $\left(F(n, m), \leqslant_{F}\right),\left(P(n, m), \leqslant_{F}\right),\left(T(n, m), \leqslant_{F}\right)$ and $\left(I(n, m), \leqslant_{F}\right)$. In particular (see Proposition 34 in the subsection below $),\left(F_{n}^{m}, \leqslant_{M}\right),\left(P_{n}^{m}, \leqslant_{M}\right),\left(T_{n}^{m}, \leqslant_{M}\right),\left(I_{n}^{m}, \leqslant_{M}\right)$ and $\left(F G_{n}^{m}, \leqslant_{M}\right),\left(P G_{n}^{m}, \leqslant_{M}\right),\left(T G_{n}^{m}, \leqslant_{M}\right),\left(I G_{n}^{m}, \leqslant_{M}\right)$ have the same sets of join- and meetirreducible elements, respectively. Thus we obtain also descriptions of join- and meetirreducible elements of $\left(F(n, m), \leqslant_{F}\right),\left(P(n, m), \leqslant_{F}\right),\left(T(n, m), \leqslant_{F}\right)$ and $\left(I(n, m), \leqslant_{F}\right)$. Next, we precisely describe structures of subposets induced by sets of join- and meetirreducible elements. Of course, taking $n=m$ we obtain analogous results for $\left(S G_{n}^{n}, \leqslant_{M}\right)$, $\left(S_{n}^{n}, \leqslant_{M}\right)$ and $\left(S(n), \leqslant_{B}\right)$.

\subsection{Basic notions and facts}

The classical construction due to $\mathrm{H}$. MacNeille order embeds a given poset $P$ in a complete lattice $L(P)$ (see [5] and also [7]). This complete lattice $L(P)$ is called the DedekindMacNeille completion of $P$ (or the completion by cuts). Recall shortly this construction. Take a poset $\left(P, \leqslant_{P}\right)$ and for each $X \subseteq P$, let $X^{u}=\left\{p \in P: \forall_{x \in X} x \leqslant_{P} p\right\}$ and $X^{l}=\left\{p \in P: \forall_{x \in X} p \leqslant_{P} x\right\}$. Next, let $L(P)=\left\{X \subseteq P: X^{u l}=X\right\}$. Then it is easy to see that the function $X \longmapsto X^{u l}$ is a closure operator, i.e., $X \subseteq X^{u l},\left(X^{u l}\right)^{u l}=X^{u l}$ and $X \subseteq Y$ implies $X^{u l} \subseteq Y^{u l}$ (see [3]). Hence $L(P)$ is a closure system, so $(L(P), \subseteq)$ is a complete lattice. Next, $\varphi_{P}: P \longrightarrow L(P)$ such that $\varphi_{P}(p)=\{p\}^{l}$, for all $p \in P$, is an embedding of $P$ into $L(P)$. Recall also that for $\left\{X_{i}\right\}_{i \in I} \subseteq L(P), \bigwedge_{i \in I} X_{i}=\left(\bigcap_{i \in I} X_{i}\right)^{u l}=$ $\bigcap_{i \in I} X_{i}$ and $\bigvee_{i \in I} X_{i}=\left(\bigcup_{i \in I} X_{i}\right)^{u l}$ (in particular, $\bigcup_{i \in I} X_{i} \subseteq \bigvee_{i \in I} X_{i}$ ).

It easily follows from the definition of the Dedekind-MacNeille completion that if posets $\left(P, \leqslant_{P}\right)$ and $\left(Q, \leqslant_{Q}\right)$ are isomorphic, then $L(P)$ and $L(Q)$ are also isomorphic.

Take a poset $\left(P, \leqslant_{P}\right)$, its dual $P^{d}$ and let $L^{l u}(P)=\left\{X \subseteq P: X^{l u}=X\right\}$. Then $L\left(P^{d}\right)=L^{l u}(P)$. Next, if $X^{u l}=X$ (respectively, $\left.X^{l u}=X\right)$, then $\left(X^{u}\right)^{l u}=X^{u}$, i.e., $X^{u} \in L^{l u}(P)$ (respectively, $\left(X^{l}\right)^{u l}=X^{l}$, i.e., $\left.X^{l} \in L(P)\right)$. Thus we have functions $L(P) \ni X \longmapsto X^{u} \in L^{l u}(P)$ and $L^{l u}(P) \ni X \longmapsto X^{l} \in L(P)$ which are invertible each other, so they are bijections. Of course, $X \subseteq Y$ implies $Y^{u} \subseteq X^{u}$ and $Y^{l} \subseteq X^{l}$. Thus these functions are anti-isomorphisms, so $L(P) \simeq\left(L\left(P^{d}\right)\right)^{d}$. Hence $L(P)^{d} \simeq L\left(P^{d}\right)$. This implies that if $P$ is self-dual (i.e., $P \simeq P^{d}$ ), then $L(P)$ is self-dual, too, because isomorphic posets have isomorphic Dedekind-MacNeille completions. Thus we have obtained 
Proposition 33. Let $P$ be a poset. Then $L(P)^{d} \simeq L\left(P^{d}\right)$. In particular, if $P$ is self-dual, then $L(P)$ is self-dual.

It is not difficult to see that the embedding $\varphi_{P}$ of a given poset $P$ into its DedekindMacNeille completion $L(P)$ preserves all joins and meets which exist in $P$ (see [[5], Theorem 7.40(ii)] for details). Next, $\varphi_{P}(P)$ is both join-dense and meet-dense in $L(P)$ (see [[5], Theorem 7.41(i)]), i.e., for each $X \in L(P)$ there are $Q, R \subseteq P$ such that $\bigvee \varphi_{P}(Q)=\bigwedge \varphi_{P}(R)=X$; it is sufficient to take $Q=X$ and $R=X^{u}$ (because $\left.\bigwedge_{r \in R}\{r\}^{l}=\bigcap_{r \in R}\{r\}^{l}=R^{l}=X^{u l}=X\right)$. Having these two facts we can prove the following result (see also [11] where another proof of this result is given for finite posets).

Proposition 34. A poset $P$ and its Dedekind-MacNeille completion $L(P)$ have the same sets of join-irreducible and meet-irreducible elements, i.e., $\operatorname{Jir}(L(P))=\varphi_{P}(\operatorname{Jir}(P))$ and $\operatorname{Mir}(L(P))=\varphi_{P}(\operatorname{Mir}(P))$.

Proof. Take $X \in \operatorname{Jir}(L(P))$. Then $\bigvee \varphi_{P}(Q)=X$ for some $Q \subseteq P$. Thus $X=\varphi_{P}(q)$ for some $q \in Q$. If $q$ would be join-reducible, then $\varphi_{P}(q)$ would be also join-reducible, because $\varphi_{P}$ preserves all joins. Thus $X \in \varphi_{P}(\operatorname{Jir}(P))$.

Now take $p \in \operatorname{Jir}(P)$ and let $\varphi_{P}(p)=\bigvee \mathcal{X}$ for some $\mathcal{X} \subseteq L(P)$. Since $\varphi_{P}(P)$ is joindense in $L(P)$, for each $X \in \mathcal{X}$ there is $Q_{X} \subseteq P$ such that $\bigvee \varphi_{P}\left(Q_{X}\right)=X$, in particular, $\bigcup \varphi_{P}\left(Q_{X}\right) \subseteq X$. Thus

$$
\bigcup\left(\varphi_{P}\left(\bigcup_{X \in \mathcal{X}} Q_{X}\right)\right)=\bigcup\left(\bigcup_{X \in \mathcal{X}} \varphi_{P}\left(Q_{X}\right)\right)=\bigcup_{X \in \mathcal{X}}\left(\bigcup \varphi_{P}\left(Q_{X}\right)\right) \subseteq \bigcup \mathcal{X}
$$

which implies

$$
\bigvee\left(\varphi_{P}\left(\bigcup_{X \in \mathcal{X}} Q_{X}\right)\right)=\left(\bigcup\left(\varphi_{P}\left(\bigcup_{X \in \mathcal{X}} Q_{X}\right)\right)\right)^{u l} \subseteq(\bigcup \mathcal{X})^{u l}=\bigvee \mathcal{X}
$$

On the other hand, $\varphi_{P}\left(Q_{X}\right) \subseteq \varphi_{P}\left(\bigcup_{X \in \mathcal{X}} Q_{X}\right)$, so $X=\bigvee \varphi_{P}\left(Q_{X}\right) \subseteq \bigvee \varphi_{P}\left(\bigcup_{X \in \mathcal{X}} Q_{X}\right)$. Hence

$$
\bigvee \mathcal{X} \subseteq \bigvee \varphi_{P}\left(\bigcup_{X \in \mathcal{X}} Q_{X}\right)
$$

Summarizing, $\varphi_{P}(p)=\bigvee \mathcal{X}=\bigvee \varphi_{P}\left(\bigcup_{X \in \mathcal{X}} Q_{X}\right)$. This implies that $p$ is the supremum of $\bigcup_{X \in \mathcal{X}} Q_{X}$ in $P$, because $\varphi_{P}$ is an isomorphism between $P$ and $\varphi_{P}(P)$. Hence $p \in \bigcup_{X \in \mathcal{X}} Q_{X}$, i.e., $p \in Q_{X_{0}}$ for some $X_{0} \in \mathcal{X}$, because $p \in \operatorname{Jir}(P)$. Then $\varphi_{P}(p) \subseteq$ $\bigvee \varphi_{P}\left(Q_{X_{0}}\right)=X_{0} \subseteq \bigvee \mathcal{X}=\varphi_{P}(p)$ (recall that $L(P)$ is partially ordered by $\subseteq$ ), so $\varphi_{P}(p)=X_{0} \in \mathcal{X}$. Thus $\varphi_{P}(p) \in \operatorname{Jir}(L(P))$.

The second equality can be obtained in the analogous way or we take the dual $P^{d}$ and use facts $\operatorname{Mir}(P)=\operatorname{Jir}\left(P^{d}\right)$ and $L\left(P^{d}\right) \simeq L(P)^{d}$.

The Dedekind-MacNeille completion is characterized in the following way.

Theorem 35 ([5], Theorem 7.41(ii)). Let a poset $P$, a complete lattice $L$ and an embedding $\varphi: P \longrightarrow L$ satisfy the following condition: for each $l \in L$, there are subsets $X, Y \subseteq P$ such that $l=\bigvee \varphi(X)=\bigwedge \varphi(Y)$. Then there is an isomorphism $\bar{\varphi}: L \longrightarrow L(P)$ of $L$ onto the Dedekind-MacNeille completion $L(P)$ of $P$ such that $\bar{\varphi} \circ \varphi=\varphi_{P}$. 
By Proposition 34, $\bar{\varphi} \circ \varphi(\operatorname{Jir}(P))=\varphi_{P}(\operatorname{Jir}(P))=\operatorname{Jir}(L(P))$, so $\varphi(\operatorname{Jir}(P))=$ $\bar{\varphi}^{-1}(\operatorname{Jir}(L(P)))=\operatorname{Jir}(L)$. In the same way we obtain $\varphi(\operatorname{Mir}(P))=\operatorname{Mir}(L)$. Summarizing,

Corollary 36. Let $P, L$ and $\varphi$ be as in Theorem 35. Then $\operatorname{Jir}(L)=\varphi(\operatorname{Jir}(P))$ and $\operatorname{Mir}(L)=\varphi(\operatorname{Mir}(P))$.

Taking $\varphi=i d_{P}$ in the above two results we obtain that if a poset $P$ is a subposet of a complete lattice $L$ such that for each $l \in L$, there are $X, Y \subseteq P$ such that $l=\bigvee X=\bigwedge Y$, then there is an isomorphism $\bar{\varphi}: L \longrightarrow L(P)$ such that $\varphi_{\mid P}=\varphi_{P}$. Moreover, $\operatorname{Jir}(L)=$ $\operatorname{Jir}(P)$ and $\operatorname{Mir}(L)=\operatorname{Mir}(P)$. In fact, in the rest of this section we will use only these simpler versions of Theorem 35 and Corollary 36.

For a finite lattice $\left(L, \leqslant_{L}\right)$, its set $\operatorname{Jir}(L)$ of all join-irreducible elements is join-dense in $\left(L, \leqslant_{L}\right)$ (i.e., each element of $L$ is the join of join-irreducible elements) and its set $\operatorname{Mir}(L)$ of all meet-irreducible elements is meet-dense in $\left(L, \leqslant_{L}\right)$ (i.e., each element of $L$ is the meet of meet-irreducible elements). Thus by the above facts we obtain the following interesting result concerning Dedekind-MacNeille completions, which will be used in the next subsections.

Corollary 37. Let $\left(L, \leqslant_{L}\right)$ be a finite lattice and $P$ be a subposet of $\left(L, \leqslant_{L}\right)$ such that $\operatorname{Jir}(L) \subseteq P$ and $\operatorname{Mir}(L) \subseteq P$. Then $\left(L, \leqslant_{L}\right) \simeq(L(P), \subseteq)$ (i.e., $L$ is isomorphic to the Dedekind-MacNeille completion of $P)$ and $\operatorname{Jir}(P)=\operatorname{Jir}(L), \operatorname{Mir}(P)=\operatorname{Mir}(L)$.

\subsection{Join- and meet-irreducible elements of finite sublattices of $\left(M_{n}^{m}(\mathbb{Z}), \leqslant_{M}\right)$}

In the paper [7] (Subsections 4.2, 4.3, 4.4 and 4.5) M. Fortin has described join- and meetirreducible elements of lattice $\left(P G_{n}^{n}, \leqslant_{M}\right)$. Now we show that his concepts and results concerning this particular case can be adopted without major changes to arbitrary finite sublattices of lattice $\left(M_{n}^{m}(\mathbb{Z}), \leqslant_{M}\right)$.

In the whole subsection $\left(L_{n}^{m}, \leqslant_{M}\right)$ denotes a finite sublattice of $\left(M_{n}^{m}(\mathbb{Z}), \leqslant_{M}\right)$ with the bottom element $\mathbf{0}_{L_{n}^{m}}$ and the top element $\mathbf{1}_{L_{n}^{m}}$. Next, for each $k=1,2, \ldots, n$ and $l=1,2, \ldots, m$, let $\pi_{(k, l)}: M_{n}^{m}(\mathbb{Z}) \longrightarrow \mathbb{Z}$ be the projection on the $(k, l)$-coordinate, i.e., $\pi_{(k, l)}(A)=A[k, l]$.

Definition 38. Let $1 \leqslant k \leqslant n, 1 \leqslant l \leqslant m$ and let $a \in \pi_{(k, l)}\left(L_{n}^{m}\right) \backslash\left\{\mathbf{0}_{L_{n}^{m}}[k, l]\right\}, b \in$ $\pi_{(k, l)}\left(L_{n}^{m}\right) \backslash\left\{\mathbf{1}_{L_{n}^{m}}[k, l]\right\}$. Then

(a) $B_{k, l, a, n \times m}^{L}=\bigwedge\left\{A \in L_{n}^{m}: A[k, l]=a\right\}$, i.e., $B_{k, l, a, n \times m}^{L}$ is the least matrix of $\left(L_{n}^{m}, \leqslant M\right)$ such that $B_{k, l, a, n \times m}^{L}[k, l]=a$.

(b) $C_{k, l, b, n \times m}^{L}=\bigvee\left\{A \in L_{n}^{m}: A[k, l]=b\right\}$, i.e., $C_{k, l, b, n \times m}^{L}$ is the greatest matrix of $\left(L_{n}^{m}, \leqslant_{M}\right)$ such that $C_{k, l, b, n \times m}^{L}[k, l]=b$.

The assumption $a, b \in \pi_{(k, l)}\left(L_{n}^{m}\right)$ implies in particular that $\mathbf{0}_{L_{n}^{m}}[k, l] \leqslant a, b \leqslant \mathbf{1}_{L_{n}^{m}}[k, l]$, because $\mathbf{0}_{L_{n}^{m}}[k, l] \leqslant A[k, l] \leqslant \mathbf{1}_{L_{n}^{m}}[k, l]$ for all matrices $A \in L_{n}^{m}$. Next, if we take $a=\mathbf{0}_{L_{n}^{m}}[k, l]$ in the above definition, then $\mathbf{0}_{L_{n}^{m}} \in\left\{A \in L_{n}^{m}: A[k, l]=a\right\}$, so $\bigwedge\{A \in$ 
$\left.L_{n}^{m}: A[k, l]=a\right\}=\mathbf{0}_{L_{n}^{m}}$. But the bottom element is not join-irreducible. Similarly, if we take $b=\mathbf{1}_{L_{n}^{m}}[k, l]$, then $\mathbf{1}_{L_{n}^{m}} \in\left\{A \in L_{n}^{m}: A[k, l]=b\right\}$, so $\bigvee\left\{A \in L_{n}^{m}: A[k, l]=a\right\}=\mathbf{1}_{L_{n}^{m}}$. But the top element is not meet-irreducible.

The following three results are proved in [7] only for the lattice $\left(P G_{n}^{n}, \leqslant_{M}\right)$ (Lemmas 4.2(1),(2) and 4.6(1),(2), Theorems 4.3 and 4.7, Theorems 4.12 and 4.13, respectively), but these proofs can be adopted without major changes for every finite sublattice of $\left(M_{n}^{m}(\mathbb{Z}), \leqslant_{M}\right)$. However, we give simpler and shorter proofs of the first and the third result. We recall also the short proof of the second result.

Lemma 39. Let $1 \leqslant k \leqslant n, 1 \leqslant l \leqslant m$ and let $a \in \pi_{(k, l)}\left(L_{n}^{m}\right) \backslash\left\{\mathbf{0}_{L_{n}^{m}}[k, l]\right\}, b \in$ $\pi_{(k, l)}\left(L_{n}^{m}\right) \backslash\left\{\mathbf{1}_{L_{n}^{m}}[k, l]\right\}$. Then for each $A \in L_{n}^{m}$,

(a) $a \leqslant A[k, l]$ iff $B_{k, l, a, n \times m}^{L} \leqslant{ }_{M} A$.

(b) $A[k, l] \leqslant b$ iff $A \leqslant{ }_{M} C_{k, l, b, n \times m}^{L}$.

Proof. The implication " $\Longleftarrow$ is obvious in the both cases.

If $a \leqslant A[k, l]$, then $\left(B_{k, l, a, n \times m}^{L} \wedge A\right)[k, l]=\min \left\{B_{k, l, a, n \times m}^{L}[k, l], A[k, l]\right\}=a$, so $B_{k, l, a, n \times m}^{L} \leqslant M\left(B_{k, l, a, n \times m}^{L} \wedge A\right) \leqslant_{M} A$.

If $A[k, l] \leqslant b$, then $\left(C_{k, l, b, n \times m}^{L} \vee A\right)[k, l]=\max \left\{C_{k, l, b, n \times m}^{L}[k, l], A[k, l]\right\}=b$, so $A \leqslant M$ $\left(C_{k, l, b, n \times m}^{L} \vee A\right) \leqslant{ }_{M} C_{k, l, b, n \times m}^{L}$.

Theorem 40. For all $A \in L_{n}^{m}$,

(a) $A=\bigvee \mathcal{A}$, where $\mathcal{A}=\left\{B_{k, l, a, n \times m}^{L}: 1 \leqslant k \leqslant n, 1 \leqslant l \leqslant m, a=A[k, l]\right.$ and $A[k, l] \neq$ $\left.\mathbf{0}_{L_{n}^{m}}[k, l]\right\}$

(b) $A=\bigwedge \mathcal{B}$, where $\mathcal{B}=\left\{C_{k, l, b, n \times m}^{L}: 1 \leqslant k \leqslant n, 1 \leqslant l \leqslant m, b=A[k, l]\right.$ and $A[k, l] \neq$ $\left.\mathbf{1}_{L_{n}^{m}}[k, l]\right\}$.

If $\mathcal{A}=\emptyset$, i.e., $A[k, l]=\mathbf{0}_{L_{n}^{m}}[k, l]$ for all pairs $(k, l)$, then $A=\mathbf{0}_{L_{n}^{m}}=\bigvee \emptyset$. Similarly, if $\mathcal{B}=\emptyset$, i.e., $A[k, l]=\mathbf{1}_{L_{n}^{m}}[k, l]$ for all pairs $(k, l)$, then $A=\mathbf{1}_{L_{n}^{m}}[k, l]=\bigwedge \emptyset$.

Proof. Let $D=\bigvee \mathcal{A}$. Then $D[k, l] \geqslant B_{k, l, A[k, l], n \times m}^{L}[k, l]=A[k, l]$ for pairs $(k, l)$ such that $A[k, l] \neq \mathbf{0}_{L_{n}^{m}}[k, l]$. Of course, $D[i, j] \geqslant \mathbf{0}_{L_{n}^{m}}[i, j]=A[i, j]$ for all other pairs $(i, j)$. Hence $A \leqslant_{M} D$. Next, if $A[k, l] \neq \mathbf{0}_{L_{n}^{m}}[k, l]$, then $B_{k, l, A[k, l], n \times m}^{L} \leqslant_{M} A$ by Lemma 39. Thus $B \leqslant_{M} A$ for all $B \in \mathcal{A}$, so $D \leqslant_{M} A$.

Let $D=\wedge \mathcal{B}$. Then $D[k, l] \leqslant C_{k, l, A[k, l], n \times m}^{L}[k, l]=A[k, l]$ for pairs $(k, l)$ such that $A[k, l] \neq \mathbf{1}_{L_{n}^{m}}[k, l]$. Of course, $D[i, j] \leqslant \mathbf{1}_{L_{n}^{m}}[i, j]=A[i, j]$ for all other pairs $(i, j)$. So $D \leqslant_{M} A$. Next, if $A[k, l] \neq \mathbf{1}_{L_{n}^{m}}[k, l]$, then $A \leqslant_{M} C_{k, l, A[k, l], n \times m}^{L}$ by Lemma 39. Thus $A \leqslant_{M} C$ for all $C \in \mathcal{B}$, so $A \leqslant_{M} D$.

\section{Theorem 41.}

$$
\begin{aligned}
& \operatorname{Jir}\left(L_{n}^{m}\right)=\left\{B_{k, l, a, n \times m}^{L}: 1 \leqslant k \leqslant n, 1 \leqslant l \leqslant m \text { and } a \in \pi_{(k, l)}\left(L_{n}^{m}\right), \quad a \neq \mathbf{0}_{L_{n}^{m}}[k, l]\right\}, \\
& \operatorname{Mir}\left(L_{n}^{m}\right)=\left\{C_{k, l, b, n \times m}^{L}: 1 \leqslant k \leqslant n, 1 \leqslant l \leqslant m \text { and } b \in \pi_{(k, l)}\left(L_{n}^{m}\right), \quad b \neq \mathbf{1}_{L_{n}^{m}}[k, l]\right\} .
\end{aligned}
$$


Proof. The inclusion " $\subseteq$ " follows from Theorem 40 in the both cases.

Since $B_{k, l, a, n \times m}^{L} \neq \mathbf{0}_{L_{n}^{m}}$, we can assume that $B_{k, l, a, n \times m}^{L}=A_{1} \vee A_{2}$ for some $A_{1}, A_{2} \in L_{n}^{m}$. Then $a=B_{k, l, a, n \times m}^{L}[k, l]=\max \left\{A_{1}[k, l], A_{2}[k, l]\right\}$. So $a=A_{i}[k, l]$, where $i=1$ or $i=2$. Hence and by Lemma 39, $B_{k, l, a, n \times m}^{L} \leqslant{ }_{M} A_{i} \leqslant_{M} A_{1} \vee A_{2}$. Thus $B_{k, l, a, n \times m}^{L}=A_{i}$, so $B_{k, l, a, n \times m}^{L}$ is join-irreducible.

Since $C_{k, l, a, n \times m}^{L} \neq \mathbf{1}_{L_{n}^{m}}$, we can assume that $C_{k, l, a, n \times m}^{L}=A_{1} \wedge A_{2}$ for some $A_{1}, A_{2} \in L_{n}^{m}$. Then $a=C_{k, l, a, n \times m}^{p}[k, l]=\min \left\{A_{1}[k, l], A_{2}[k, l]\right\}$. So $a=A_{i}[k, l]$, where $i=1$ or $i=2$. Hence and by Lemma 39, $A_{1} \wedge A_{2} \leqslant{ }_{M} A_{i} \leqslant{ }_{M} C_{k, l, a, n \times m}^{L}$. Thus $C_{k, l, a, n \times m}^{L}=A_{i}$, so $C_{k, l, a, n \times m}^{L}$ is meet-irreducible.

\subsection{Join-irreducible elements of the lattice $\left(P G_{n}^{m}, \leqslant_{M}\right)$}

In this and the next subsections we will use Theorem 41 to show interesting informations about join-irreducible and meet-irreducible elements in lattices $\left(F G_{n}^{m}, \leqslant_{M}\right),\left(P G_{n}^{m}, \leqslant_{M}\right)$, $\left(T G_{n}^{m}, \leqslant_{M}\right),\left(I G_{n}^{m}, \leqslant_{M}\right)$ and posets $\left(F_{n}^{m}, \leqslant_{M}\right),\left(P_{n}^{m}, \leqslant_{M}\right),\left(T_{n}^{m}, \leqslant_{M}\right),\left(I_{n}^{m}, \leqslant_{M}\right)$, thus also posets $\left(F(n, m), \leqslant_{F}\right),\left(P(n, m), \leqslant_{F}\right),\left(T(n, m), \leqslant_{F}\right),\left(I(n, m), \leqslant_{F}\right)$.

We start with the lattice $\left(P G_{n}^{m}, \leqslant_{M}\right)$. By the definition of the set $P G_{n}^{m}$ (see Definition 9 ) and properties of the bottom $\mathbf{0}_{n}^{m}$ and the top element $\nabla_{n}^{m}$ of the lattice $\left(P G_{n}^{m}, \leqslant_{M}\right)$ (see the last part of Subsection 2.3) we obtain $\pi_{(k, l)}\left(P G_{n}^{m}\right)=\left\{\mathbf{0}_{n}^{m}[k, l], \mathbf{0}_{n}^{m}[k, l]+1, \ldots, \nabla_{n}^{m}[k, l]\right\}$ for all $k=1,2, \ldots, n$ and $l=1,2, \ldots, m$. Hence and by Theorem 41 we have that all joinirreducible elements of the lattice $\left(P G_{n}^{m}, \leqslant M\right)$ are of the form $B_{k, l, a, n \times m}^{P G}$, where $1 \leqslant k \leqslant n$, $1 \leqslant l \leqslant m$ and $1=\mathbf{0}_{n}^{m}[k, l]+1 \leqslant a \leqslant \nabla_{n}^{m}[k, l]=\min \{k, m+1-l\} \leqslant \min \{n, m\}$. These matrices will be denoted by $B_{k, l, a, n \times m}^{p}$ to simplify notation. In other words, we have

$$
\operatorname{Jir}\left(P G_{n}^{m}\right)=\left\{B_{k, l, a, n \times m}^{p}: 1 \leqslant k \leqslant n, 1 \leqslant l \leqslant m \text { and } 1 \leqslant a \leqslant \min \{k, m+1-l\}\right\} .
$$

Since $B_{k, l, a, n \times m}^{p}$ is the least matrix of $\left(P G_{n}^{m}, \leqslant_{M}\right)$ with the entry $a$ in the position $(k, l)$, we obtain that the matrix $B_{k, l, a, n \times m}^{p}$ looks as follows (note that $l+a-1 \leqslant m$ )

$$
\left[\begin{array}{cccccccccccc}
0 & \multicolumn{1}{c}{l} & \multicolumn{1}{c}{l+a} & \multicolumn{1}{c}{l} & m \\
\vdots & \vdots & \vdots & \vdots & \vdots & \vdots & \vdots & \vdots & \vdots & \vdots & \vdots & \vdots \\
0 & 0 & \cdots & 0 & 0 & 0 & \cdots & 0 & 0 & 0 & \cdots & 0 \\
1 & 1 & \cdots & 1 & 0 & 0 & \cdots & 0 & 0 & 0 & \cdots & 0 \\
2 & 2 & \cdots & 2 & 1 & 0 & \cdots & 0 & 0 & 0 & \cdots & 0 \\
\vdots & \vdots & \vdots & \vdots & \vdots & \vdots & \vdots & \vdots & \vdots & \vdots & \vdots & \vdots \\
a-1 & a-1 & \cdots & a-1 & a-2 & a-3 & \cdots & 1 & 0 & 0 & \cdots & 0 \\
a & a & \cdots & a & a-1 & a-2 & \cdots & 2 & 1 & 0 & \cdots & 0 \\
a & a & \cdots & a & a-1 & a-2 & \cdots & 2 & 1 & 0 & \cdots & 0 \\
\vdots & \vdots & \vdots & \vdots & \vdots & \vdots & \vdots & \vdots & \vdots & \vdots & \vdots & \vdots \\
a & a & \cdots & a & a-1 & a-2 & \cdots & 2 & 1 & 0 & \cdots & 0
\end{array}\right]_{n}
$$


Thus the matrix $O^{r}\left(B_{k, l, a, n \times m}^{p}\right)$ equals

$$
\left[\begin{array}{cccccccccccc}
0 & 0 & \cdots & 0 & 0 & 0 & \cdots & 0 & 0 & 0 & \cdots & 0 \\
\vdots & \vdots & \vdots & \vdots & \vdots & \vdots & \vdots & \vdots & \vdots & \vdots & \vdots & \vdots \\
0 & 0 & \cdots & 0 & 0 & 0 & \cdots & 0 & 0 & 0 & \cdots & 0 \\
1 & 1 & \cdots & 1 & 0 & 0 & \cdots & 0 & 0 & 0 & \cdots & 0 \\
1 & 1 & \cdots & 1 & 1 & 0 & \cdots & 0 & 0 & 0 & \cdots & 0 \\
\vdots & \vdots & \vdots & \vdots & \vdots & \vdots & \vdots & \vdots & \vdots & \vdots & \vdots & \vdots \\
1 & 1 & \cdots & 1 & 1 & 1 & \cdots & 1 & 0 & 0 & \cdots & 0 \\
1 & 1 & \cdots & 1 & 1 & 1 & \cdots & 1 & 1 & 0 & \cdots & 0 \\
0 & 0 & \cdots & 0 & 0 & 0 & \cdots & 0 & 0 & 0 & \cdots & 0 \\
\vdots & \vdots & \vdots & \vdots & \vdots & \vdots & \vdots & \vdots & \vdots & \vdots & \vdots & \vdots \\
0 & 0 & \cdots & 0 & 0 & 0 & \cdots & 0 & 0 & 0 & \cdots & 0
\end{array}\right]_{n}{ }_{n}
$$

Hence (see also Remark 8) we obtain the following result, which is proved in [7], Lemma $4.2(3)$, for $n=m$. The proof given in [7] can be easily adopted for arbitrary $n, m \in \mathbb{N}$, but our proof is simpler.

Proposition 42. Let $1 \leqslant k \leqslant n, 1 \leqslant l \leqslant m$ and $1 \leqslant a \leqslant \min \{k, m+1-l\}$. Then

(a) $B_{k, l, a, n \times m}^{p} \in P_{n}^{m}$.

(b) $f_{B_{k, l, a, n \times m}^{p}}=\left(\begin{array}{ccccccccccc}1 & 2 & \ldots & k-a & k-a+1 & k-a+2 & \ldots & k & k+1 & \ldots & n \\ 0 & 0 & \ldots & 0 & l & l+1 & \ldots & l+a-1 & 0 & \ldots & 0\end{array}\right)$.

Of course, for $f=\left(\begin{array}{ccccccccccc}1 & 2 & \ldots & r-1 & r & r+1 & \ldots & s & s+1 & \ldots & n \\ 0 & 0 & \ldots & 0 & x & x+1 & \ldots & x+s-r & 0 & \ldots & 0\end{array}\right)$, where $1 \leqslant r \leqslant$ $s \leqslant n$ and $1 \leqslant x \leqslant m-s+r$, we have $f=f_{B_{s, x, s-r+1, n \times m}^{p}}$. So $M(f)=B_{s, x, s-r+1, n \times m}^{p}$; this fact follows also from the equality $M^{c}(f)=O^{r}\left(B_{s, x, s-r+1, n \times m}^{p}\right)$. Note that $1 \leqslant s-r+1 \leqslant$ $\min \{s, m+1-x\}$.

Now we precisely describe the structure of the poset $\left(\operatorname{Jir}\left(P G_{n}^{m}\right), \leqslant_{M}\right)$. To this purpose we first prove the following technical lemma.

Lemma 43. Let $1 \leqslant k_{1}, k_{2} \leqslant n, 1 \leqslant l_{1}, l_{2} \leqslant m$ and $1 \leqslant a_{1} \leqslant \min \left\{k_{1}, m+1-l_{1}\right\}$, $1 \leqslant a_{2} \leqslant \min \left\{k_{2}, m+1-l_{2}\right\}$. Then

$$
B_{k_{1}, l_{1}, a_{1}, n \times m}^{p} \leqslant{ }_{M} B_{k_{2}, l_{2}, a_{2}, n \times m}^{p} \quad \text { iff } \quad\left\{\begin{array}{l}
k_{1}-a_{1} \geqslant k_{2}-a_{2} \\
l_{1}+a_{1} \leqslant l_{2}+a_{2} \\
a_{1} \leqslant a_{2} \\
a_{1}+l_{1}-k_{1} \leqslant a_{2}+l_{2}-k_{2}
\end{array}\right.
$$

$$
B_{k_{1}, l_{1}, a_{1}, n \times m}^{p}=B_{k_{2}, l_{2}, a_{2}, n \times m}^{p} \quad \text { iff } \quad\left(k_{1}, l_{1}, a_{1}\right)=\left(k_{2}, l_{2}, a_{2}\right) .
$$


Proof. Note first that (b) is implied by (a). This fact may be also easily obtained by using the form of matrices $O^{r}\left(B_{k, l, a, n \times m}^{p}\right)$.

(a): Let $C_{1}=B_{k_{1}, l_{1}, a_{1}, n \times m}^{p}$ and $C_{2}=B_{k_{2}, l_{2}, a_{2}, n \times m}^{p}$. Observe that the first non-zero rows in $C_{1}$ and $C_{2}$ lie in the positions $k_{1}-a_{1}+1$ and $k_{2}-a_{2}+1$, respectively, and similarly, the last non-zero columns in $C_{1}$ and $C_{2}$ lie in the positions $l_{1}+a_{1}-1$ and $l_{2}+a_{2}-1$, respectively.

By Lemma 39 we have the equivalence

$$
C_{1} \leqslant{ }_{M} C_{2} \text { iff } a_{1} \leqslant C_{2}\left[k_{1}, l_{1}\right] .
$$

We divide our proof into the following four cases:

(a.1): $k_{1} \geqslant k_{2}$ and $l_{1} \leqslant l_{2}$. Then $C_{2}\left[k_{1}, l_{1}\right]=C_{2}\left[k_{2}, l_{2}\right]=a_{2}$, so $C_{1} \leqslant{ }_{M} C_{2}$ iff $a_{1} \leqslant a_{2}$. All other inequalities (from the right-hand side of our equivalence) are implied by the proved inequality $a_{1} \leqslant a_{2}$ and our assumptions of this case. The similar situation will hold in the next cases, therefore we will not explicit formulate it later.

(a.2): $k_{1} \leqslant k_{2}$ and $l_{1} \leqslant l_{2}$. Then $C_{2}\left[k_{1}, l_{1}\right]=C_{2}\left[k_{1}, l_{2}\right]=C_{2}\left[k_{2}-\left(k_{2}-k_{1}\right), l_{2}\right]=$ $\max \left\{C_{2}\left[k_{2}, l_{2}\right]-\left(k_{2}-k_{1}\right), 0\right\}=\max \left\{a_{2}-\left(k_{2}-k_{1}\right), 0\right\}$, so $C_{1} \leqslant{ }_{M} C_{2}$ iff $a_{1} \leqslant \max \left\{a_{2}-\right.$ $\left.\left(k_{2}-k_{1}\right), 0\right\}$. The last inequality is equivalent to $a_{1} \leqslant a_{2}-\left(k_{2}-k_{1}\right)$, because $a_{1} \geqslant 1$. Thus $C_{1} \leqslant{ }_{M} C_{2}$ iff $a_{1}-k_{1} \leqslant a_{2}-k_{2}$.

(a.3): $k_{1} \geqslant k_{2}$ and $l_{1} \geqslant l_{2}$. Then $C_{2}\left[k_{1}, l_{1}\right]=C_{2}\left[k_{2}, l_{1}\right]=C_{2}\left[k_{2}, l_{2}+\left(l_{1}-l_{2}\right)\right]=$ $\max \left\{C_{2}\left[k_{2}, l_{2}\right]-\left(l_{1}-l_{2}\right), 0\right\}=\max \left\{a_{2}-\left(l_{1}-l_{2}\right), 0\right\}$, so $C_{1} \leqslant{ }_{M} C_{2}$ iff $a_{1} \leqslant \max \left\{a_{2}-\right.$ $\left.\left(l_{1}-l_{2}\right), 0\right\}$. The last inequality is equivalent to $a_{1} \leqslant a_{2}-\left(l_{1}-l_{2}\right)$, because $a_{1} \geqslant 1$. Thus $C_{1} \leqslant{ }_{M} C_{2}$ iff $a_{1}+l_{1} \leqslant a_{2}+l_{2}$.

(a.4): $k_{1} \leqslant k_{2}$ and $l_{1} \geqslant l_{2}$. Then $C_{2}\left[k_{1}, l_{1}\right]=C_{2}\left[k_{2}-\left(k_{2}-k_{1}\right), l_{2}+\left(l_{1}-l_{2}\right)\right]=$ $\max \left\{C_{2}\left[k_{2}, l_{2}\right]-\left(k_{2}-k_{1}\right)-\left(l_{1}-l_{2}\right), 0\right\}=\max \left\{a_{2}-\left(k_{2}-k_{1}\right)-\left(l_{1}-l_{2}\right), 0\right\}$, so $C_{1} \leqslant{ }_{M} C_{2}$ iff $a_{1} \leqslant \max \left\{a_{2}-\left(k_{2}-k_{1}\right)-\left(l_{1}-l_{2}\right), 0\right\}$. The last inequality is equivalent to $a_{1} \leqslant a_{2}-\left(k_{2}-k_{1}\right)-\left(l_{1}-l_{2}\right)$, because $a_{1} \geqslant 1$. Thus $C_{1} \leqslant{ }_{M} C_{2}$ iff $a_{1}+l_{1}-k_{1} \leqslant a_{2}+l_{2}-k_{2}$.

Theorem 44. $\left(\operatorname{Jir}\left(P G_{n}^{m}\right), \leqslant_{M}\right)$ is isomorphic to a subposet of the poset $\left(\mathbb{N}^{4}, \leqslant_{\text {prod }}\right)$ induced by the set $T_{n, m}=\left\{(x, y, z, t) \in \mathbb{N}^{4}: 1 \leqslant x \leqslant n, 1 \leqslant y \leqslant m, 1 \leqslant z \leqslant\right.$ $\min \{x, y\}$ and $t=x+y-z\}$. Moreover, this isomorphism is given by the function

$$
B_{k, l, a, n \times m}^{p} \longmapsto(n-k+a, l+a-1, a, a-k+l+n-1) .
$$

Note that $T_{n, m} \subseteq\{1,2, \ldots, n\} \times\{1,2, \ldots, m\} \times\{1,2, \ldots, \min \{n, m\}\} \times\{1,2, \ldots, n+m-1\}$.

Proof. Having Lemma 43(b) we can take a function $\Psi: \operatorname{Jir}\left(P G_{n}^{m}\right) \longrightarrow \mathbb{N}^{4}$ such that

$$
\Psi\left(B_{k, l, a, n \times m}^{p}\right)=(n-k+a, l+a-1, a, a+l-k)+(0,0,0, n-1) .
$$

Lemma $43(\mathrm{a})$ implies that $\Psi$ is an embedding of $\left(\operatorname{Jir}\left(P G_{n}^{m}\right), \leqslant_{M}\right)$ into $\left(\mathbb{N}^{4}, \leqslant_{\text {prod }}\right)$. Next, for each $B_{k, l, a, n \times m}^{p}$ we have that $1 \leqslant a \leqslant n-k+a \leqslant n$ and $1 \leqslant a \leqslant l+a-1 \leqslant m$, because $a \leqslant \min \{k, m+1-l\}$. Moreover, $a+l-k+n-1=(n-k+a)+(l+a-1)-a$. Hence $\Psi\left(\operatorname{Jir}\left(P G_{n}^{m}\right)\right) \subseteq T_{n, m}$. On the other hand, take $(x, y, z, t) \in T_{n, m}$. Applying inequalities which define the set $T_{n, m}$, it can be shown (simple details are left to the reader) that

$1 \leqslant n-x+z \leqslant n, \quad 1 \leqslant y-z+1 \leqslant m$ and $1 \leqslant z \leqslant \min \{n-x+z, m+1-(y-z+1)\}$. 
Thus we can take the matrix $B_{n-x+z, y-z+1, z, n \times m}^{p}$. Of course, $\Psi\left(B_{n-x+z, y-z+1, z, n \times m}^{p}\right)=$ $(x, y, z, x+y-z)=(x, y, z, t)$. Hence $T_{n, m} \subseteq \Psi\left(\operatorname{Jir}\left(P G_{n}^{m}\right)\right)$, because $(x, y, z, t) \in T_{n, m}$ was arbitrarily chosen.

Proposition 45. $\left|\operatorname{Jir}\left(P G_{n}^{m}\right)\right|=\frac{\alpha(\alpha+1)(2 \alpha+1)}{6}+\frac{\alpha(\alpha+1)}{2} \cdot(\beta-\alpha)$, where $\alpha=\min \{n, m\}$ and $\beta=\max \{n, m\}$. In particular, $\left|\operatorname{Jir}\left(P G_{n}^{n}\right)\right|=\frac{n(n+1)(2 n+1)}{6}$.

Proof. By Lemma 43(b) we have

$$
\left|\operatorname{Jir}\left(P G_{n}^{m}\right)\right|=|S|
$$

where $S=\left\{(k, l, a) \in \mathbb{N}^{3}: 1 \leqslant k \leqslant n, 1 \leqslant l \leqslant m, 1 \leqslant a \leqslant \alpha, a \leqslant k\right.$ and $\left.l \leqslant m-a+1\right\}$.

Next, $|\{(k, l, a) \in S: a=i\}|=(n-i+1) \cdot(m-i+1)$ for $1 \leqslant i \leqslant \alpha$. So

$$
|S|=\sum_{i=1}^{\min \{n, m\}}(n-i+1) \cdot(m-i+1) .
$$

Assume $n \leqslant m$ (the case $m \leqslant n$ is analogous, it is sufficient to replace $m$ by $n$ and vice versa). Then $\sum_{i=1}^{n}(n-i+1) \cdot(m-i+1)=\sum_{i=1}^{n}(n-i+1) \cdot((m-n)+(n-i+1))=$ $\sum_{i=1}^{n}\left((n-i+1) \cdot(m-n)+(n-i+1)^{2}\right)=\sum_{i=1}^{n}((n-i+1) \cdot(m-n))+\sum_{i=1}^{n}(n-i+1)^{2}=$ $(m-n) \cdot(1+2+3+\cdots+n)+\left(1^{2}+2^{2}+3^{2}+\cdots+n^{2}\right)=\frac{n(n+1)}{2} \cdot(m-n)+\frac{n(n+1)(2 n+1)}{6}$.

Since $\left(P G_{n}^{m}, \leqslant_{M}\right)$ is a finite distributive lattice, it is isomorphic to the lattice of all order ideals of $\operatorname{Jir}\left(P G_{n}^{m}\right)$ (see [4] and Subsection 1.1). Hence and by Theorem 44 (see also Subsection 1.1 for the second part of this fact) we obtain

Corollary 46. $\left(P G_{n}^{m}, \leqslant_{M}\right) \simeq\left(O I\left(T_{n, m}\right), \subseteq\right)$. In particular, $\left|P G_{n}^{m}\right|$ is equal to the cardinality of the family of all anti-chains of $\left(T_{n, m}, \leqslant\right.$ prod $)$.

\subsection{Meet-irreducible elements of the lattice $\left(P G_{n}^{m}, \leqslant_{M}\right)$}

At the beginning of Subsection 3.3 we have shown $\pi_{(k, l)}\left(P G_{n}^{m}\right)=\left\{\mathbf{0}_{n}^{m}[k, l], \mathbf{0}_{n}^{m}[k, l]+\right.$ $\left.1, \ldots, \nabla_{n}^{m}[k, l]\right\}$ for all $k=1,2, \ldots, n$ and $l=1,2, \ldots, m$. Thus by Theorem 41 we have that all meet-irreducible elements of the lattice $\left(P G_{n}^{m}, \leqslant_{M}\right)$ are of the form $C_{k, l, a, n \times m}^{P G}$, where $1 \leqslant k \leqslant n, 1 \leqslant l \leqslant m$ and $0=\mathbf{0}_{n}^{m}[k, l] \leqslant a \leqslant \nabla_{n}^{m}[k, l]-1=\min \{k, m+1-l\}-1 \leqslant$ $\min \{n, m\}-1$. These matrices will be denoted by $C_{k, l, a, n \times m}^{p}$ to simplify notation. In other words, we have

$$
\operatorname{Mir}\left(P G_{n}^{m}\right)=\left\{C_{k, l, a, n \times m}^{p}: 1 \leqslant k \leqslant n, 1 \leqslant l \leqslant m \text { and } 0 \leqslant a \leqslant \min \{k, m+1-l\}-1\right\} .
$$

Unfortunately, matrices of the form $C_{k, l, a, n \times m}^{p}$ have more complicated structure than matrices of the form $B_{k, l, a, n \times m}^{p}$. Therefore the proof of an analogous result to Proposition 42 is technically more complicated. 
Take $A=C_{k, l, a, n \times m}^{p}$ and observe that the first $k+2$ rows of the matrix $A$ look as follows (note that $l<m-a+1$ ):

$$
\left[\begin{array}{cccccccccccccc}
\multicolumn{1}{c}{} & \multicolumn{1}{c}{l} & \multicolumn{1}{c}{m-a} & m-a+1 & & & & & \\
1 & \cdots & 1 & 1 & 1 & \cdots & 1 & 1 & 1 & 1 & 1 & \cdots & 1 & 1 \\
2 & \cdots & 2 & 2 & 2 & \cdots & 2 & 2 & 2 & 2 & 2 & \cdots & 2 & 1 \\
\vdots & \vdots & \vdots & \vdots & \vdots & \vdots & \vdots & \vdots & \vdots & \vdots & \vdots & \vdots & \vdots & \vdots \\
a-1 & \cdots & a-1 & a-1 & a-1 & \cdots & a-1 & a-1 & a-1 & a-1 & a-2 & \cdots & 2 & 1 \\
a & \cdots & a & a & a & \cdots & a & a & a & a-1 & a-2 & \cdots & 2 & 1 \\
a+1 & \cdots & a+1 & a+1 & a & \cdots & a & a & a & a-1 & a-2 & \cdots & 2 & 1 \\
a+2 & \cdots & a+2 & a+1 & a & \cdots & a & a & a & a-1 & a-2 & \cdots & 2 & 1 \\
\vdots & \vdots & \vdots & \vdots & \vdots & \vdots & \vdots & \vdots & \vdots & \vdots & \vdots & \vdots & \vdots & \vdots \\
x & \cdots & a+2 & a+1 & \underline{a} & \cdots & a & a & a & a-1 & a-2 & \cdots & 2 & 1 \\
x+1 & \cdots & a+3 & a+2 & a+1 & \cdots & a+1 & a+1 & a & a-1 & a-2 & \cdots & 2 & 1 \\
x+2 & \cdots & a+4 & a+3 & a+2 & \cdots & a+2 & a+1 & a & a-1 & a-2 & \cdots & 2 & 1 \\
\vdots & \vdots & \vdots & \vdots & \vdots & \vdots & \vdots & \vdots & \vdots & \vdots & \vdots & \vdots & \vdots & \vdots
\end{array}\right]
$$

where $x=a+\min \{l-1, k-a\}$.

Of course, for $a=1$ we have $A[i, j]=1$ for all $i \leqslant k$ and $j \geqslant l$. Similarly, if $a=0$, then $A[i, j]=0$ for all $i \leqslant k$ and $j \geqslant l$.

Thus the first $k+2$ rows of the matrix $O^{r}(A)$ look as follows:

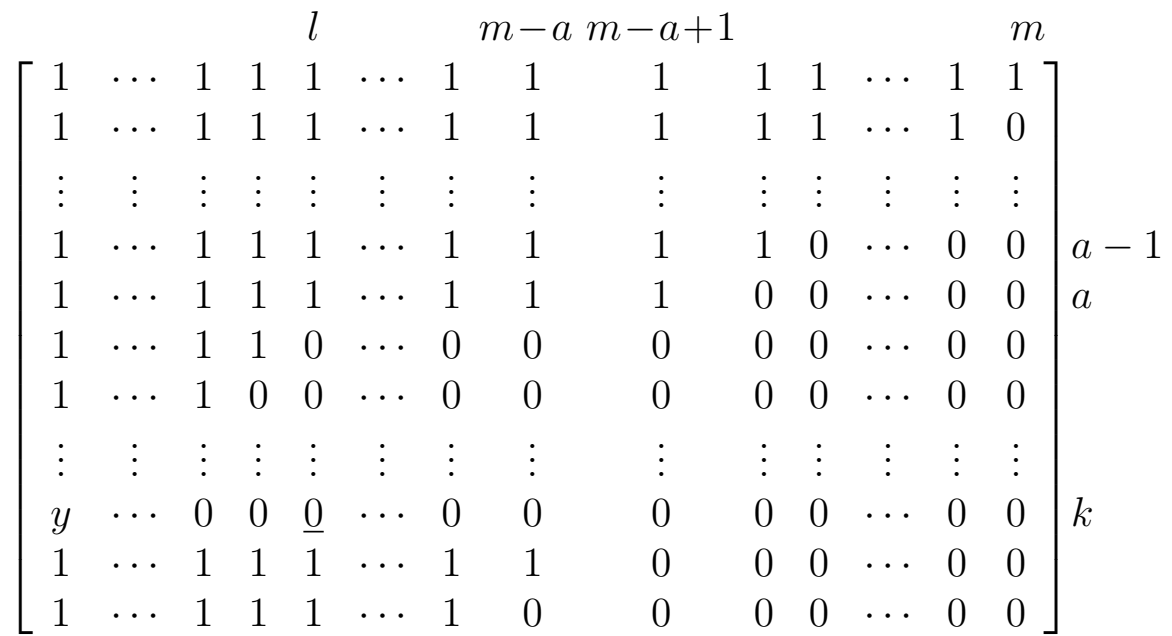

where $y=0$ if $l \leqslant k-a$ and $y=1$ if $k-a \leqslant l-1$.

If $k-a \leqslant l-1$, then the maximal number $j$ such that $A[k, j]=1$ is equal $l-(k-a)=$ $a+l-k$. If $l \leqslant k-a$, then the first row of $A$ which consists of only zeros has the number $a+l$. Thus the first $k+2$ rows of $A$ correspond to one of the following two functions: If $l \leqslant k-a$, then $\left(\begin{array}{ccccccccccc}1 & \ldots & a & a+1 & \ldots & a+l-1 & a+l & \ldots & k & k+1 & k+2 \\ m & \ldots & m-a+1 & l-1 & \ldots & 1 & 0 & \ldots & 0 & m-a & m-a-1\end{array} \mid\right.$. If $k-a \leqslant l-1$, then $\left(\begin{array}{cccccccc}1 & \ldots & a & a+1 & \ldots & k & k+1 & k+2 \\ m & \ldots & m-a+1 & l-1 & \ldots & l-(k-a) & m-a & m-a-1\end{array} \mid\right.$.

To describe the rest rows of matrices $A$ and $O^{r}(A)$ we consider three cases. 
Case 1: $m-a-(n-k) \geqslant l-1$.

Then the last $n-k$ rows of $A$ looks as follows:

$$
\left[\begin{array}{ccccccccccccccc}
x+1 & \ldots & a+2 & a+1 & \ldots & a+1 & \ldots & a+1 & a+1 & a & a-1 & \ldots & 2 & 1 \\
x+2 & \ldots & a+3 & a+2 & \ldots & a+2 & \ldots & a+2 & a+1 & a & a-1 & \ldots & 2 & 1 \\
\vdots & \ldots & \vdots & \vdots & \ldots & \vdots & \ldots & \vdots & \vdots & \vdots & \vdots & \ldots & \vdots & \vdots \\
\alpha & \ldots & \beta+1 & \beta & \ldots & \beta & \cdots & a+2 & a+1 & a & a-1 & \cdots & 2 & 1
\end{array}\right] n
$$

(1) it is the $l$-th column; ${ }^{(2)}$ it is $(m-a+1-(n-k))$-th column (note that $m-a+1-$ $(n-k) \geqslant l) ; \quad{ }^{(3)}$ it is $(m-a+1)$-th column. Next, $\alpha=\min \{x+n-k, m\}, \beta=a+n-k$.

Take $1 \leqslant i \leqslant n-k$. Then first,

$A[k+i, l]=a+i, A[k+i, m-a+1-i]=a+i$ and $A[k+i, m-a+1-i+1]=a+i-1$.

Secondly, the part of the $k+i$-th row of $A$ between the $l$-th and $(m-a+1-i)$-th columns is non-empty and each entry of this part equals $a+i$. Thirdly, the last $m-(m-a+1-i)=$ $a+i-1$ elements of this row forms a strictly decreasing sequence ending by 1 . Moreover, for each $1 \leqslant j \leqslant l$, the last $n-k$ elements of the $j$-th column form a strictly increasing sequence. Thus the last $n-k$ rows of $O^{r}(A)$ look as follows:

$$
\left[\begin{array}{cccccccccccccc}
1 & \ldots & 1 & 1 & \ldots & 1 & \ldots & 1 & 1 & 0 & 0 & \ldots & 0 & 0 \\
1 & \ldots & 1 & 1 & \ldots & 1 & \ldots & 1 & 0 & 0 & 0 & \ldots & 0 & 0 \\
\vdots & \cdots & \vdots & \vdots & \cdots & \vdots & \cdots & \vdots & \vdots & \vdots & \vdots & \cdots & \vdots & \vdots \\
1 & \cdots & 1 & 1 & \cdots & 1 & \cdots & 0 & 0 & 0 & 0 & \cdots & 0 & 0
\end{array}\right] n
$$

Thus in this case $A \in P_{n}^{m}$ and the last $n-k$ rows of $A$ corresponds to the following function:

$$
\left.\mid \begin{array}{ccccc}
k+1 & k+2 & k+3 & \ldots & n \\
m-a & m-a-1 & m-a-2 & \ldots & m-a-(n-k-1)
\end{array}\right),
$$

note that $m-a-(n-k-1) \geqslant l$.

Case 2: $m-a-(n-k) \leqslant l-2$.

Let $r=m-a+1-l, s=m-a+1-(n-k)$ and $z=l-s=l-(m-a+1-(n-k))$. Note that $r \leqslant n-k-1$. Then for all $1 \leqslant i \leqslant r$,

$$
A[k+i, l]=A[k+i, l+1]=A[k+i, l+2]=\ldots=A[k+i, m-a+1-i]=a+i
$$

and for all $r+1 \leqslant i \leqslant n-k$,

$$
A[k+i, l]=A[k+r, l] .
$$

Say very informally, these equalities follow from the fact that the segment connecting points $(n, s)$ and $(k, m-a+1)$ intersects with the $l$-th column of $A$ in the point $(k+r, l)$ 
(note that $s$ may be negative integer, so $(n, s)$ may lie to the left to the first column of $A$ ). Hence the last $n-k$ rows of $A$ look as follows:

$$
\left[\begin{array}{cccccccccccc}
\cdots & a+3 & a+2 & a+1 & \cdots & a+1 & a+1 & a & a-1 & \cdots & 2 & 1 \\
\cdots & a+4 & a+3 & a+2 & \cdots & a+2 & a+1 & a & a-1 & \cdots & 2 & 1 \\
\vdots & \vdots & \vdots & \vdots & \vdots & \vdots & \vdots & \vdots & \vdots & \vdots & \vdots & \vdots \\
\cdots & a+r+1 & a+r & a+r-1 & \cdots & a+2 & a+1 & a & a-1 & \cdots & 2 & 1 \\
\cdots & a+r+2 & a+r+1 & a+r & \cdots & a+2 & a+1 & a & a-1 & \cdots & 2 & 1 \\
\cdots & a+r+2 & a+r+1 & a+r & \cdots & a+2 & a+1 & a & a-1 & \cdots & 2 & 1 \\
\vdots & \vdots & \vdots & \vdots & \vdots & \vdots & \vdots & \vdots & \vdots & \vdots & \vdots & \vdots \\
\cdots & a+r+2 & a+r+1 & a+r & \cdots & a+2 & a+1 & a & a-1 & \cdots & 2 & 1
\end{array}\right]_{n}
$$

(1) it is the $l$-th column; ${ }^{(2)}$ it is $(m-a+1)$-th column.

Case 2.1: $l-1 \leqslant k-a$

Then $x=a+\min \{l-1, k-a\}=a+l-1$. Thus the first $l$ entries of the $k$ th row form the following strictly decreasing sequence $a+l-1, a+l-2, \ldots, a+1, a$. Hence, for each $i=1,2, \ldots, r$, the first $l$ entries of the $(k+i)$-th row are equal to $a+l-1+i, a+l-2+i, \ldots, a+1+i, a+i$, respectively. Moreover, for all $i=r, r+1, \ldots, n-k$, the first $l$ entries of the $(k+i)$-th row are equal to $a+l-1+r, a+l-2+r, \ldots, a+r+1, a+r$, respectively, because the last $n-(k+r)$ entries of the $l$-th column are equal to $a+r$. These facts imply that the last $n-k$ rows of $O^{r}(A)$ look as follows:

$$
\left[\begin{array}{ccccccccccccc}
1 & \cdots & 1 & 1 & 1 & \cdots & 1 & 1 & 0 & 0 & \cdots & 0 & 0 \\
1 & \cdots & 1 & 1 & 1 & \cdots & 1 & 0 & 0 & 0 & \cdots & 0 & 0 \\
\vdots & \vdots & \vdots & \vdots & \vdots & \vdots & \vdots & \vdots & \vdots & \vdots & \vdots & \vdots & \vdots \\
1 & \cdots & 1 & 1 & 1 & \cdots & 0 & 0 & 0 & 0 & \cdots & 0 & 0 \\
1 & \cdots & 1 & 1 & 1 & \cdots & 0 & 0 & 0 & 0 & \cdots & 0 & 0 \\
0 & \cdots & 0 & 0 & 0 & \cdots & 0 & 0 & 0 & 0 & \cdots & 0 & 0 \\
\vdots & \vdots & \vdots & \vdots & \vdots & \vdots & \vdots & \vdots & \vdots & \vdots & \vdots & \vdots & \vdots \\
0 & \cdots & 0 & 0 & 0 & \cdots & 0 & 0 & 0 & 0 & \cdots & 0 & 0
\end{array}\right]_{n}
$$

(1) it is the $l$-th column; ${ }^{(2)}$ it is $(m-a+1)$-th column.

Thus in this case $A \in P_{n}^{m}$ and the last $n-k$ rows of the matrix $A$ correspond to the following function:

$$
\left.\begin{array}{cccccccc}
k+1 & k+2 & \ldots & k+m-a+1-l-1 & k+m-a+1-l & k+m-a+1-l+1 & \ldots & n \\
m-a & m-a-1 & \ldots & l+1 & l & 0 & \ldots & 0
\end{array}\right),
$$

note that $k+m-a+1-l=k+r \leqslant k+n-k-1=n-1$.

Case 2.2: $k-a \leqslant l-2$. 
Then $l-(k-a) \geqslant 2$ and $x=a+\min \{l-1, k-a\}=k$. Thus the first $l$ entries of the $k$-th row form the following decreasing sequence $\underbrace{k, k, \ldots, k}_{l-(k-a)}, k-1, \ldots, a+1, a$. Hence, for each $1 \leqslant i \leqslant r$, the first $l$ entries of the $(k+i)$-th row are equal to $\underbrace{k+i, k+i, \ldots, k+i}_{l-(k-a)}, k-$ $1+i, \ldots, a+1+i, a+i$, respectively. Thus for each $1 \leqslant i \leqslant \min \{l-(k-a)-1, n-(k+r)\}$, the first $l$ entries of the $(k+r+i)$-th row are equal to $\underbrace{k+r+i, k+r+i, \ldots, k+r+i}_{l-(k-a)-i}, k+$ $r+i-1, \ldots, a+r+1, a+r$, respectively, because the last $n-(k+r)$ entries of the $l$-th column are equal to $a+r$. Moreover, if $l-(k-a) \leqslant n-(k+r)$ (which is equivalent with $n-m-1 \geqslant 0)$, then for each $l-(k-a) \leqslant i \leqslant n-(k+r)$, the $(k+r+i)$-th row is a strictly decreasing sequence ending by 1 . So in this case the last $n-(k+r+l-(k-a)-1)=n-m$ rows of $A$ are equal. All these facts imply that the last $n-k$ rows of $O^{r}(A)$ look as follows:

$$
\left[\begin{array}{ccccccccccccccccc}
1 & \cdots & 1 & 1 & 1 & \cdots & 1 & 1 & 1 & \cdots & 1 & 1 & 0 & 0 & \cdots & 0 & 0 \\
1 & \cdots & 1 & 1 & 1 & \cdots & 1 & 1 & 1 & \cdots & 1 & 0 & 0 & 0 & \cdots & 0 & 0 \\
\vdots & \vdots & \vdots & \vdots & \vdots & \vdots & \vdots & \vdots & \vdots & \vdots & \vdots & \vdots & \vdots & \vdots & \vdots & \vdots & \vdots \\
1 & \cdots & 1 & 1 & 1 & \cdots & 1 & 1 & 1 & \cdots & 0 & 0 & 0 & 0 & \cdots & 0 & 0 \\
1 & \cdots & 1 & 1 & 1 & \cdots & 1 & 1 & 1 & \cdots & 0 & 0 & 0 & 0 & \cdots & 0 & 0 \\
1 & \cdots & 1 & 1 & 0 & \cdots & 0 & 0 & 0 & \cdots & 0 & 0 & 0 & 0 & \cdots & 0 & 0 \\
1 & \cdots & 1 & 0 & 0 & \cdots & 0 & 0 & 0 & \cdots & 0 & 0 & 0 & 0 & \cdots & 0 & 0 \\
\vdots & \vdots & \vdots & \vdots & \vdots & \vdots & \vdots & \vdots & \vdots & \vdots & \vdots & \vdots & \vdots & \vdots & \vdots & \vdots & \vdots \\
1 & \cdots & 0 & 0 & 0 & \cdots & 0 & 0 & 0 & \cdots & 0 & 0 & 0 & 0 & \cdots & 0 & 0 \\
0 & \cdots & 0 & 0 & 0 & \cdots & 0 & 0 & 0 & \cdots & 0 & 0 & 0 & 0 & \cdots & 0 & 0 \\
\vdots & \vdots & \vdots & \vdots & \vdots & \vdots & \vdots & \vdots & \vdots & \vdots & \vdots & \vdots & \vdots & \vdots & \vdots & \vdots & \vdots \\
0 & \cdots & 0 & 0 & 0 & \cdots & 0 & 0 & 0 & \cdots & 0 & 0 & 0 & 0 & \cdots & 0 & 0
\end{array}\right]_{n}
$$

(0) it is the $(l-(k-a)-1)$-th column; (1) it is the $l$-th column; $\left.\quad{ }^{2}\right)$ it is $(m-a+1)$-th column. Next, ${ }^{(a)} k+r=k+m-a+1-l$ and ${ }^{(b)} k+r+l-(k-a)-1=m$.

Thus in this case $A \in P_{n}^{m}$ and the last $n-k$ rows of the matrix $A$ correspond to one of the following three functions:

If $m+1 \leqslant n$, then

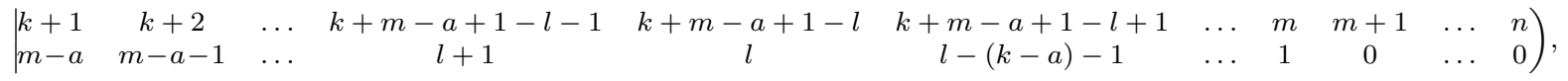

because $(k+m-a+1-l)+(l-k+a-1)=m$. Our assumption $k-a \leqslant l-2$ implies $l-(k-a)-1 \geqslant 1$ and $k+m-a+1-l+1 \leqslant m$.

If $n \leqslant m$, then

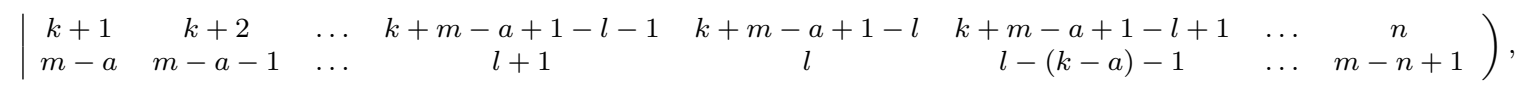

because $l-(k-a)-(n-(k+m-a+1-l))=m-n+1$. Our assumption $m-a-(n-k) \leqslant l-2$ implies $k+m-a+1-l \leqslant n-1$.

Summarizing we have proved the following result (see also Remark 8). 
Proposition 47. Let $1 \leqslant k \leqslant n, 1 \leqslant l \leqslant m$ and $0 \leqslant a \leqslant \min \{k, m+1-l\}-1$. Then

(a) $C_{k, l, a, n \times m}^{p} \in P_{n}^{m}$.

(b) $f_{C_{k, l, a, n \times m}^{p}}$ is such that

(b.1) if $m-n+k-a \geqslant l-1$ and $l \leqslant k-a$, then $f_{C_{k, l, a, n \times m}^{p}}=\left(\begin{array}{cccccc}1 & \ldots & a & a+1 & \ldots \\ m & \ldots & m-a+1 & l-1 & \ldots\end{array}\right.$ $\left.\begin{array}{ccccccc}a+l & \ldots & k & k+1 & k+2 & \ldots & n \\ 0 & \ldots & 0 & m-a & m-a-1 & \ldots & m-n+k-a+1\end{array}\right)$ (note that $a+l \leqslant k$ and $m-n+k-a+1 \geqslant l$ ),

(b.2) if $m-n+k-a \geqslant l-1$ and $k-a \leqslant l-1$ (in particular, $n \leqslant m$ ), then $f_{C_{k, l, a, n \times m}^{p}}=$

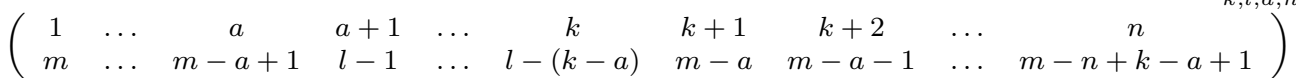
(note that $l-(k-a) \geqslant 1$ and $m-n+k-a+1 \geqslant l$ ),

(b.3) if $m-n+k-a \leqslant l-2$ and $l \leqslant k-a$ (in particular, $m \leqslant n-2$ ), then $f_{C_{k, l, a, n \times m}^{p}}=$ $\left(\begin{array}{cccccccccccc}1 & \ldots & a & a+1 & \ldots & a+l & \ldots & k & k+1 & k+2 & \ldots & m+k-a-l+1 \\ m & \ldots & m-a+1 & l-1 & \ldots & 0 & \ldots & 0 & m-a & m-a-1 & \ldots & l\end{array}\right.$ $\left.\begin{array}{ccc}m+k-a-l+2 & \ldots & n \\ 0 & \ldots & 0\end{array}\right)$

(note that $a+l \leqslant k$ and $m+k-a-l+2 \leqslant n$ ),

(b.4) if $m-n+k-a \leqslant l-2$ and $l-1=k-a$ (equivalently, $m \leqslant n-1$ and $l-1=$ $k-a)$, then $f_{C_{k, l, a, n \times m}^{p}}=\left(\begin{array}{ccccccccc}1 & \ldots & a & a+1 & \ldots & k & k+1 & k+2 & \ldots \\ m & \ldots & m-a+1 & l-1 & \ldots & 1 & m-a & m-a-1 & \ldots\end{array}\right.$ $\left.\begin{array}{cccc}m+k-a-l+1 & m+k-a-l+2 & \ldots & n \\ l & 0 & \ldots & 0\end{array}\right)$

(note that $m+k-a-l+2 \leqslant n)$,

(b.5) if $m+1 \leqslant n, m-n+k-a \leqslant l-2$ and $k-a \leqslant l-2$ (equivalently, $m \leqslant n-1$ and $k-a \leqslant l-2$; moreover, these two conditions imply $m-n+k-a \leqslant l-3)$, then $f_{C_{k, l, a, n \times m}^{p}}=\left(\begin{array}{ccccccccc}1 & \ldots & a & a+1 & \ldots & k & k+1 & k+2 & \ldots \\ m & \ldots & m-a+1 & l-1 & \ldots & l-(k-a) & m-a & m-a-1 & \ldots\end{array}\right.$ $\left.\begin{array}{ccccccc}m+k-a-l+1 & m+k-a-l+2 & \ldots & m & m+1 & \ldots & n \\ l & l-(k-a)-1 & \ldots & 1 & 0 & \ldots & 0\end{array}\right)$

(note that $l-(k-a) \geqslant 2$ and $m+k-a-l+2 \leqslant m)$,

(b.6) if $n \leqslant m, m-n+k-a \leqslant l-2$ and $k-a \leqslant l-2$ (equivalently, $n \leqslant m$ and $m-n+$ $k-a \leqslant l-2)$, then $f_{C_{k, l, a, n \times m}^{p}}=\left(\begin{array}{ccccccc}1 & \ldots & a & a+1 & \ldots & k & k+1 \\ m & \ldots & m-a+1 & l-1 & \ldots & l-(k-a) & m-a\end{array}\right.$ $\left.\begin{array}{cccccc}k+2 & \ldots & m+k-a-l+1 & m+k-a-l+2 & \ldots & n \\ m-a-1 & \ldots & l & l-(k-a)-1 & \ldots & m-n+1\end{array}\right)$ (note that $l-(k-a) \geqslant 2$ and $m-n+1 \geqslant 1)$.

The proof of the particular case of the above result for $n=m$ is sketched in [7] (Subsection 4.5, Examples 4.18, 4.19, 4.20). Of course, this particular case has a much simpler form and easily follows from Proposition 47. More precisely, if $n=m$, then assumptions of (b.3), (b.4), (b.5) are not satisfied, so by (b.1), (b.2) and (b.6) we obtain the following fact.

Corollary $48([7])$. Let $1 \leqslant k, l \leqslant n$ and $0 \leqslant a \leqslant \min \{k, n+1-l\}-1$. Then 
(a) if $l \leqslant k-a$, then $f_{C_{k, l, a, n \times n}^{p}}=\left(\begin{array}{ccccccccccc}1 & 2 & \ldots & a & a+1 & a+2 & \ldots & a+l-1 & a+l & \ldots \\ n & n-1 & \ldots & n-a+1 & l-1 & l-2 & \ldots & 1 & 0 & \ldots\end{array}\right.$ $\left.\begin{array}{ccccc}k & k+1 & k+2 & \ldots & n \\ 0 & n-a & n-a-1 & \ldots & k-a+1\end{array}\right)$, note that $k-a+1 \geqslant l+1$,

(b) if $k-a=l-1$, then $f_{C_{k, l, a, n \times n}^{p}}=\left(\begin{array}{ccccccccccc}1 & 2 & \ldots & a & a+1 & a+2 & \ldots & k & k+1 \\ n & n-1 & \ldots & n-a+1 & l-1 & l-2 & \ldots & 1 & n-a\end{array}\right.$ $\left.\begin{array}{cccc}k+2 & k+3 & \ldots & n \\ n-a-1 & n-a-2 & \ldots & l\end{array}\right)$,

(c) if $k-a \leqslant l-2$, then $f_{C_{k, l, a, n \times n}^{p}}=\left(\begin{array}{ccccccccc}1 & 2 & \ldots & a & a+1 & a+2 & \ldots & k \\ n & n-1 & \ldots & n-a+1 & l-1 & l-1 & \ldots & l-(k-a)\end{array}\right.$ $\left.\begin{array}{cccccccc}k+1 & k+2 & \ldots & n+k-a-l+1 & n+k-a-l+2 & n+k-a-l+3 & \ldots & n \\ n-a & n-a-1 & \ldots & l & l-(k-a)-1 & l-(k-a)-2 & \ldots & 1\end{array}\right)$, note that $l-(k-a) \geqslant 2$ and $n+k-a-l+1 \leqslant n-1$.

Of course, (b) is a particular case of (c).

Now we precisely describe the structure of the poset $\left(\operatorname{Mir}\left(P G_{n}^{m}\right), \leqslant M\right)$. To this purpose we first prove the following technical lemma.

Lemma 49. Let $1 \leqslant k_{1}, k_{2} \leqslant n, 1 \leqslant l_{1}, l_{2} \leqslant m$ and $0 \leqslant a_{1} \leqslant \min \left\{k_{1}, m+1-l_{1}\right\}-1$, $0 \leqslant a_{2} \leqslant \min \left\{k_{2}, m+1-l_{2}\right\}-1$. Then

$$
C_{k_{1}, l_{1}, a_{1}, n \times m}^{p} \leqslant C_{k_{2}, l_{2}, a_{2}, n \times m}^{p} \quad \text { iff }\left\{\begin{array}{l}
k_{1}-a_{1} \geqslant k_{2}-a_{2} \\
l_{1}+a_{1} \leqslant l_{2}+a_{2} \\
a_{1} \leqslant a_{2} \\
a_{1}+l_{1}-k_{1} \leqslant a_{2}+l_{2}-k_{2}
\end{array}\right.
$$

$$
C_{k_{1}, l_{1}, a_{1}, n \times m}^{p}=C_{k_{2}, l_{2}, a_{2}, n \times m}^{p} \quad \text { iff } \quad\left(k_{1}, l_{1}, a_{1}\right)=\left(k_{2}, l_{2}, a_{2}\right) .
$$

Proof. Note first that (b) is implied by (a). This fact may be also obtained by using the form of matrices $O^{r}\left(C_{k, l, a, n \times m}^{p}\right)$, which is described in the proof of Proposition 47. But it is a very complicated method in this case.

(a): We will apply the description of matrices $C_{k, l, a, n \times m}^{p}$ given in the proof of Proposition 47. Our proof is similar to the proof of Theorem 44, but unfortunately technically more complicated (because matrices of the form $C_{k, l, a, n \times m}^{p}$ have more complicated structure than matrices of the form $\left.B_{k, l, a, n \times m}^{p}\right)$.

Let $C_{1}=C_{k_{1}, l_{1}, a_{1}, n \times m}^{p}$ and $C_{2}=C_{k_{2}, l_{2}, a_{2}, n \times m}^{p}$. By Lemma 39 we have the equivalence

$$
C_{1} \leqslant{ }_{M} C_{2} \text { iff } C_{1}\left[k_{2}, l_{2}\right] \leqslant a_{2} .
$$

We divide our proof into the following several cases:

(a.1): $k_{2} \leqslant a_{1}$ or $l_{2} \geqslant m-a_{1}+1$. If $k_{2}+l_{2} \leqslant m+1$ (i.e., the position $\left(k_{2}, l_{2}\right)$ lies to the left from the line connecting positions $\left(a_{1}, m-a_{1}+1\right)$ and $\left.(1, m)\right)$, then $C_{1}\left[k_{2}, l_{2}\right]=k_{2} \geqslant a_{2}+1$. Similarly, if $k_{2}+l_{2} \geqslant m+1$ (i.e., $\left(k_{2}, l_{2}\right)$ lies to the right from the line connecting $\left(a_{1}, m-a_{1}+1\right)$ and $\left.(1, m)\right)$, then $C_{1}\left[k_{2}, l_{2}\right]=m-l_{2}+1 \geqslant a_{2}+1$. In both these cases $C_{2}$ cannot be greater than or equal to $C_{1}$ for any value of $a_{2}$. 
(a.2): $k_{1} \geqslant k_{2} \geqslant a_{1}+1$ and $l_{1} \leqslant l_{2} \leqslant m-a_{1}$. Then $C_{1}\left[k_{2}, l_{2}\right]=C_{1}\left[k_{1}, l_{1}\right]=a_{1}$, so $C_{1} \leqslant{ }_{M} C_{2}$ iff $a_{1} \leqslant a_{2}$. All other inequalities (from the right-hand side of the first equivalence) are implied by the proved inequality $a_{1} \leqslant a_{2}$ and our assumptions of this case. Similar facts will hold in the next cases, therefore we will not explicit formulate their later.

(a.3): $k_{1} \geqslant k_{2} \geqslant a_{1}+1, l_{1} \geqslant l_{2}$ and $k_{2}+l_{2} \leqslant a_{1}+l_{1}$ (i.e., the position $\left(k_{2}, l_{2}\right)$ lies to the left from the line passing through positions $\left(a_{1}, l_{1}\right)$ and $\left.\left(a_{1}+1, l_{1}-1\right)\right)$. Then $C_{1}\left[k_{2}, l_{2}\right]=k_{2} \geqslant a_{2}+1$.

(a.4): $k_{1} \geqslant k_{2} \geqslant a_{1}+1, l_{1} \geqslant l_{2}$ and $k_{2}+l_{2}>a_{1}+l_{1}$ (i.e., $\left(k_{2}, l_{2}\right)$ lies to the right from the line passing through $\left(a_{1}, l_{1}\right)$ and $\left.\left(a_{1}+1, l_{1}-1\right)\right)$. Then $C_{1}\left[k_{2}, l_{2}\right]=a_{1}+l_{1}-l_{2}$, so $C_{1} \leqslant{ }_{M} C_{2}$ iff $a_{1}+l_{1} \leqslant a_{2}+l_{2}$.

(a.5): $k_{1} \leqslant k_{2}, l_{1} \leqslant l_{2}$ and $k_{2}+l_{2}<k_{1}+m-a_{1}+1$ (i.e., the position $\left(k_{2}, l_{2}\right)$ lies to the left from the line passing through positions $\left(k_{1}, m-a_{1}+1\right)$ and $\left.\left(k_{1}+1, m-a_{1}\right)\right)$. Then $C_{1}\left[k_{2}, l_{2}\right]=a_{1}+k_{2}-k_{1}$, so $C_{1} \leqslant{ }_{M} C_{2}$ iff $a_{1}-k_{1} \leqslant a_{2}-k_{2}$ iff $k_{1}-a_{1} \geqslant k_{2}-a_{2}$.

(a.6): $k_{1} \leqslant k_{2}, l_{1} \leqslant l_{2}$ and $k_{2}+l_{2} \geqslant k_{1}+m-a_{1}+1$ (i.e., $\left(k_{2}, l_{2}\right)$ lies to the right from the line passing through $\left(k_{1}, m-a_{1}+1\right)$ and $\left.\left(k_{1}+1, m-a_{1}\right)\right)$. Then $C_{1}\left[k_{2}, l_{2}\right]=m-l_{2}+1 \geqslant a_{2}+1$. (a.7): $k_{1} \leqslant k_{2}, l_{1} \geqslant l_{2}$. Take lines $K$ and $L$ passing through positions $\left(a_{1}, l_{1}\right),\left(a_{1}+1, l_{1}-1\right)$ and $\left(k_{1}, m-a_{1}+1\right),\left(k_{1}+1, m-a_{1}\right)$, respectively. Next, let $i$ be a non-negative integer such that $\left(k_{1}, i\right)$ is the common position of $K$ and the $k_{1}$-th row if such a position exists, otherwise $i=0$. Similarly, let $j$ be a non-negative integer such that $\left(j, l_{1}\right)$ is a common position of $L$ and the $l_{1}$-th column if such a position exists, otherwise $j=m+1$. Then we have the following four cases (of course, some of this cases do not hold if $i=0$ or $j=m+1)$ :

If $k_{2} \leqslant j$ and $k_{2}+l_{2}>k_{1}+i$ (i.e., the position $\left(k_{2}, l_{2}\right)$ lies to the right from the line passing through positions $\left(k_{1}, i\right)$ and $\left.\left(k_{1}+1, i-1\right)\right)$, then $C_{1}\left[k_{2}, l_{2}\right]=a_{1}+\left(k_{2}-k_{1}\right)+\left(l_{1}-l_{2}\right)$. Hence $C_{1} \leqslant{ }_{M} C_{2}$ iff $a_{1}+\left(k_{2}-k_{1}\right)+\left(l_{1}-l_{2}\right) \leqslant a_{2}$ iff $a_{1}+l_{1}-k_{1} \geqslant a_{2}+l_{2}-k_{2}$.

If $k_{2} \leqslant j$ and $k_{2}+l_{2} \leqslant k_{1}+i$ (i.e., the position $\left(k_{2}, l_{2}\right)$ lies to the left from the line passing through positions $\left(k_{1}, i\right)$ and $\left.\left(k_{1}+1, i-1\right)\right)$, then $C_{1}\left[k_{2}, l_{2}\right]=k_{2} \geqslant a_{2}+1$.

If $k_{2} \geqslant j$ and $k_{2}+l_{2} \geqslant k_{1}+i$ (i.e., the position $\left(k_{2}, l_{2}\right)$ lies to the right from the line passing through positions $\left(k_{1}, i\right)$ and $\left.\left(k_{1}+1, i-1\right)\right)$, then $C_{1}\left[k_{2}, l_{2}\right]=m-l_{2}+1 \geqslant a_{2}+1$. If $k_{2} \geqslant j$ and $k_{2}+l_{2} \leqslant k_{1}+i$ (i.e., the position $\left(k_{2}, l_{2}\right)$ lies to the left from the line passing through positions $\left(k_{1}, i\right)$ and $\left.\left(k_{1}+1, i-1\right)\right)$, then $C_{1}\left[k_{2}, l_{2}\right]=k_{2} \geqslant a_{2}+1$.

Theorem 50. $\left(\operatorname{Mir}\left(P G_{n}^{m}\right), \leqslant_{M}\right)$ is isomorphic to the poset $\left(T_{n, m}, \leqslant_{\text {prod }}\right)$ defined in Theorem 44 , recall that $\left(T_{n, m}, \leqslant\right.$ prod $)$ is a subposet of the poset $\left(\mathbb{N}^{4}, \leqslant\right.$ prod $)$ such that $T_{n, m}=$ $\left\{(x, y, z, t) \in \mathbb{N}^{4}: 1 \leqslant x \leqslant n, 1 \leqslant y \leqslant m, 1 \leqslant z \leqslant \min \{x, y\}\right.$ and $\left.t=x+y-z\right\}$. Moreover, this isomorphism is given by the function

$$
C_{k, l, a, n \times m}^{p} \longmapsto(n-k+a+1, l+a, a+1, a-k+l+n) .
$$

Proof. Having Lemma 49(b) we can take a function $\Psi: \operatorname{Mir}\left(P G_{n}^{m}\right) \longrightarrow \mathbb{N}^{4}$ such that

$$
\Psi\left(C_{k, l, a, n \times m}^{p}\right)=(n-k+a+1, l+a, a+1, a-k+l)+(0,0,0, n) .
$$

Lemma 49 (a) implies that $\Psi$ is an embedding of $\left(\operatorname{Mir}\left(P G_{n}^{m}\right), \leqslant_{M}\right)$ into $\left(\mathbb{N}^{4}, \leqslant_{\text {prod }}\right)$. For each $C_{k, l, a, n \times m}^{p}$ we have $1 \leqslant a+1 \leqslant n-k+a+1 \leqslant n$ and $1 \leqslant a+1 \leqslant l+a \leqslant m$, because 
$a \leqslant \min \{k, m+1-l\}-1$. Next, $a-k+l+n=(n-k+a+1)+(l+a)-(a+1)$. Hence $\Psi\left(\operatorname{Mir}\left(P G_{n}^{m}\right)\right) \subseteq T_{n, m}$. On the other hand, take $(x, y, z, t) \in T_{n, m}$. By the last part of the proof of Theorem 44 we have that

$1 \leqslant n-x+z \leqslant n, \quad 1 \leqslant y-z+1 \leqslant m$ and $0 \leqslant z-1 \leqslant \min \{n-x+z, m+1-(y-z+1)\}-1$.

Thus we can that the matrix $C_{n-x+z, y-z+1, z-1, n \times m}^{p}$. Of course, $\Psi\left(C_{n-x+z, y-z+1, z-1, n \times m}^{p}\right)=$ $(x, y, z, x+y-z)=(x, y, z, t)$. Hence $T_{n, m} \subseteq \Psi\left(\operatorname{Mir}\left(P G_{n}^{m}\right)\right)$, because $(x, y, z, t) \in T_{n, m}$ was arbitrarily chosen.

Proposition 51. $\left|\operatorname{Mir}\left(P G_{n}^{m}\right)\right|=\frac{\alpha(\alpha+1)(2 \alpha+1)}{6}+\frac{\alpha(\alpha+1)}{2} \cdot(\beta-\alpha)$, where $\alpha=\min \{n, m\}$ and $\beta=\max \{n, m\}$. In particular, $\left|\operatorname{Mir}\left(P G_{n}^{n}\right)\right|=\frac{n(n+1)(2 n+1)}{6}$.

Proof. By Lemma 49(b) we have $\left|\operatorname{Mir}\left(P G_{n}^{m}\right)\right|=\mid\left\{(k, l, a) \in \mathbb{N}^{3}: 1 \leqslant k \leqslant n, 1 \leqslant l \leqslant\right.$ $m, 0 \leqslant a \leqslant \alpha-1, a \leqslant k-1$ and $l \leqslant m-a\}|=|\left\{(k, l, a+1) \in \mathbb{N}^{3}: 1 \leqslant k \leqslant n, 1 \leqslant l \leqslant\right.$ $m, 1 \leqslant a+1 \leqslant \alpha, a+1 \leqslant k$ and $l \leqslant m-(a+1)+1\} \mid$. Thus by the proof of Proposition 45 we have our result.

By Theorems 44 and 50 we have that functions $B_{k, l, a, n \times m}^{p} \longmapsto(n-k+a, l+a-1, a, a-$ $k+l+n-1)$ and $C_{k, l, a-1, n \times m}^{p} \longmapsto(n-k+(a-1)+1, l+(a-1),(a-1)+1,(a-1)-k+l+n)=$ $(n-k+a, l+a-1, a, a-k+l+n-1)$, where $1 \leqslant a \leqslant \min \{k, m+1-l\}$, are isomorphisms of $\left(\operatorname{Jir}\left(P G_{n}^{m}\right), \leqslant_{M}\right)$ and $\left(\operatorname{Mir}\left(P G_{n}^{m}\right), \leqslant_{M}\right)$ onto $\left(T_{n, m}, \leqslant_{\text {prod }}\right)$, respectively. Hence obtain the following result.

Corollary 52. Posets $\left(\operatorname{Jir}\left(P G_{n}^{m}\right), \leqslant_{M}\right)$ and $\left(\operatorname{Mir}\left(P G_{n}^{m}\right), \leqslant_{M}\right)$ are isomorphic. Moreover, this isomorphism is given by the function $B_{k, l, a, n \times m}^{p} \longmapsto C_{k, l, a-1, n \times m}^{p}$ for all $1 \leqslant k \leqslant n$, $1 \leqslant l \leqslant m$ and $1 \leqslant a \leqslant \min \{k, m+1-l\}$.

Since $\left(P G_{n}^{m}, \leqslant_{M}\right)$ is a finite distributive lattice, its dual $\left(P G_{n}^{m}, \leqslant_{M}^{d}\right)$ is isomorphic to the lattice of all order filters of $\operatorname{Mir}\left(P G_{n}^{m}\right)$ (see [4] and Subsection 1.1). Hence and by Theorem 50 we obtain

Corollary 53. $\left(P G_{n}^{m}, \leqslant_{M}^{d}\right) \simeq\left(O F\left(T_{n, m}\right), \subseteq\right)$.

By this corollary (see also Subsection 1.1) we obtain another proof of the fact that $\left|P G_{n}^{m}\right|$ is equal to the cardinality of the family of all anti-chains of $\left(T, \leqslant_{\text {prod }}\right)$ (see Corollary 46).

\subsection{Dedekind-MacNeille completions of posets $\left(P_{n}^{m}, \leqslant_{M}\right)$ and $\left(P(n, m), \leqslant_{F}\right)$}

By Corollary 37 and Propositions 42(a), 47(a) we obtain the following description of Dedekind-MacNeille completion of the poset $\left(P_{n}^{m}, \leqslant_{M}\right)$ (see also Proposition 10).

Theorem 54. $\left(L\left(P_{n}^{m}\right), \subseteq\right) \simeq\left(P G_{n}^{m}, \leqslant_{M}\right)$ (i.e., the Dedekind-MacNeille completion of the poset $\left(P_{n}^{m}, \leqslant_{M}\right)$ is isomorphic to the lattice $\left.\left(P G_{n}^{m}, \leqslant_{M}\right)\right)$, in particular, $\left(L\left(P_{n}^{m}\right), \subseteq\right)$ is a finite distributive lattice. Moreover, $\operatorname{Jir}\left(P_{n}^{m}\right)=\operatorname{Jir}\left(P G_{n}^{m}\right)$ and $\operatorname{Mir}\left(P_{n}^{m}\right)=\operatorname{Mir}\left(P G_{n}^{m}\right)$. 
Since the function $f \longmapsto M(f)$ is an isomorphism between posets $\left(P(n, m), \leqslant_{F}\right)$ and $\left(P_{n}^{m}, \leqslant_{M}\right)$, the following properties of the poset $\left(P(n, m), \leqslant_{F}\right)$ are obtained by Theorem 54 and Propositions 42(b), 47(b).

\section{Corollary 55.}

(a) $(L(P(n, m)), \subseteq) \simeq\left(P G_{n}^{m}, \leqslant_{M}\right)$, i.e., the Dedekind-MacNeille completion of the poset $\left(P(n, m), \leqslant_{F}\right)$ is isomorphic to $\left(P G_{n}^{m}, \leqslant_{M}\right)$. In particular, $(L(P(n, m)), \subseteq)$ is a finite distributive lattice.

(b) $\operatorname{Jir}(P(n, m))=\left\{f_{B_{k, l, a, n \times m}^{p}}: 1 \leqslant k \leqslant n, 1 \leqslant l \leqslant m\right.$ and $1 \leqslant a \leqslant \min \{k, m-l+$ $1\}\}=\left\{\left(\begin{array}{ccccccccccc}1 & 2 & \ldots & r-1 & r & r+1 & \ldots & s & s+1 & \ldots & n \\ 0 & 0 & \ldots & 0 & x & x+1 & \ldots & x+s-r & 0 & \ldots & 0\end{array}\right): 1 \leqslant r \leqslant s \leqslant n, 1 \leqslant\right.$ $x \leqslant m-s+r\}$.

(c) $\operatorname{Mir}(P(n, m))=\left\{f_{C_{k, l, a, n \times m}^{p}}: 1 \leqslant k \leqslant n, 1 \leqslant l \leqslant m\right.$ and $0 \leqslant a \leqslant \min \{k, m-l+$ $1\}-1\}=\{f \in P(n, m): f$ has one of the six forms given in Proposition $47(b)\}$.

By Corollary 55, Theorems 44, 50 and Corollary 52 we obtain the following result which describes the structure of subposets of $\left(P(n, m), \leqslant_{F}\right)$ induced by join- and meetirreducible elements.

Corollary 56. Let $T_{n, m}=\left\{(x, y, z, t) \in \mathbb{N}^{4}: 1 \leqslant x \leqslant n, 1 \leqslant y \leqslant m, 1 \leqslant z \leqslant\right.$ $\min \{x, y\}$ and $t=x+y-z\}$. Then

(a) $\left(\operatorname{Jir}(P(n, m)), \leqslant_{F}\right)$ and $\left(\operatorname{Mir}(P(n, m)), \leqslant_{F}\right)$ are isomorphic to $\left(T_{n, m}, \leqslant_{\text {prod }}\right)$. Moreover, these isomorphisms are given by functions

$$
\Theta_{1}: f_{B_{k, l, a, n \times m}^{p}} \longmapsto(n-k+a, l+a-1, a, a-k+l+n-1)
$$

and

$$
\Theta_{2}: f_{C_{k, l, a, n \times m}^{p}} \longmapsto(n-k+a+1, l+a, a+1, a-k+l+n) .
$$

(b) The function $f_{B_{k, l, a, n \times m}^{p}} \longmapsto f_{C_{k, l, a-1, n \times m}^{p}}$ is an isomorphism of $\left(\operatorname{Jir}(P(n, m)), \leqslant_{F}\right)$ onto $\left(\operatorname{Mir}(P(n, m)), \leqslant_{F}\right)$.

Since join-irreducible elements of the poset $\left(P(n, m), \leqslant_{F}\right)$ have a simple structure, the function $\Theta_{1}$ can be described without using the isomorphism $B_{k, l, a, n \times m}^{p} \longmapsto f_{B_{k, l, a, n \times m}^{p}}$ between posets $\left(\operatorname{Jir}\left(P G_{n}^{m}\right), \leqslant_{M}\right)$ and $\left(\operatorname{Jir}(P(n, m)), \leqslant_{F}\right)$. Take an arbitrary function

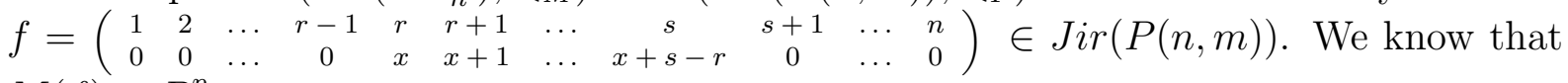
$M(f)=B_{s, x, s-r+1, n \times m}^{p}$, so

$$
\begin{gathered}
\Theta_{1}(f)=\Theta_{1}\left(f_{B_{s, x, s-r+1, n \times m}^{p}}\right)= \\
(n-s+(s-r+1), x+(s-r+1)-1, s-r+1,(s-r+1)-s+x+n-1)= \\
(n-r+1, x-r+s, s-r+1, x-r+n)=(n-r+1, f(s), s-r+1, f(r)-r+n) .
\end{gathered}
$$


Unfortunately, it is not possible to obtain such a formula for the function $\Theta_{2}$, because meet-irreducible elements of $\left(P(n, m), \leqslant_{F}\right)$ have a much more complicated structure than join-irreducible elements (see Proposition 47). In fact, several partial formulas can be given, each of them is defined on some part of $\operatorname{Mir}(P(n, m))$ and together give the function $\Theta_{2}$.

\subsection{Join-irreducible elements of the lattice $\left(F G_{n}^{m}, \leqslant_{M}\right)$}

By the definition of the set $F G_{n}^{m}$ (see Definition 9 ) and properties of the bottom $\mathbf{0}_{n}^{m}$ and the top element $\mathbf{1}_{F_{n}^{m}}$ of the lattice $\left(F G_{n}^{m}, \leqslant_{M}\right)$ (see the last part of Subsection 2.3) we obtain that $\pi_{(k, l)}\left(F G_{n}^{m}\right)=\left\{\mathbf{0}_{n}^{m}[k, l], \mathbf{0}_{n}^{m}[k, l]+1, \ldots, \mathbf{1}_{F_{n}^{m}}[k, l]\right\}$ for all $k=1,2, \ldots, n$ and $l=1,2, \ldots, m$. Hence and by Theorem 41 we have that all join-irreducible elements of the lattice $\left(F G_{n}^{m}, \leqslant M\right)$ are of the form $B_{k, l, a, n \times m}^{F G}$, where $1 \leqslant k \leqslant n, 1 \leqslant l \leqslant m$ and $1=\mathbf{0}_{n}^{m}[k, l]+1 \leqslant a \leqslant \mathbf{1}_{F_{n}^{m}}[k, l]=k$. These matrices will be denoted by $B_{k, l, a, n \times m}^{f}$ to simplify notation. In other words, we have

$$
\operatorname{Jir}\left(F G_{n}^{m}\right)=\left\{B_{k, l, a, n \times m}^{f}: 1 \leqslant k \leqslant n, 1 \leqslant l \leqslant m \text { and } 1 \leqslant a \leqslant k\right\} .
$$

Since $B_{k, l, a, n \times m}^{f}$ is the least matrix of $F G_{n}^{m}$ with the entry $a$ in the position $(k, l)$, we obtain

$$
B_{k, l, a, n \times m}^{f}[i, j]= \begin{cases}0 & \text { if } 1 \leqslant i \leqslant k-a \\ 0 & \text { if } l+1 \leqslant j \leqslant m \\ a+i-k & \text { if } k-a \leqslant i \leqslant k \text { and } 1 \leqslant j \leqslant l \\ a & \text { if } k \leqslant i \leqslant n \text { and } 1 \leqslant j \leqslant l\end{cases}
$$

i.e.,

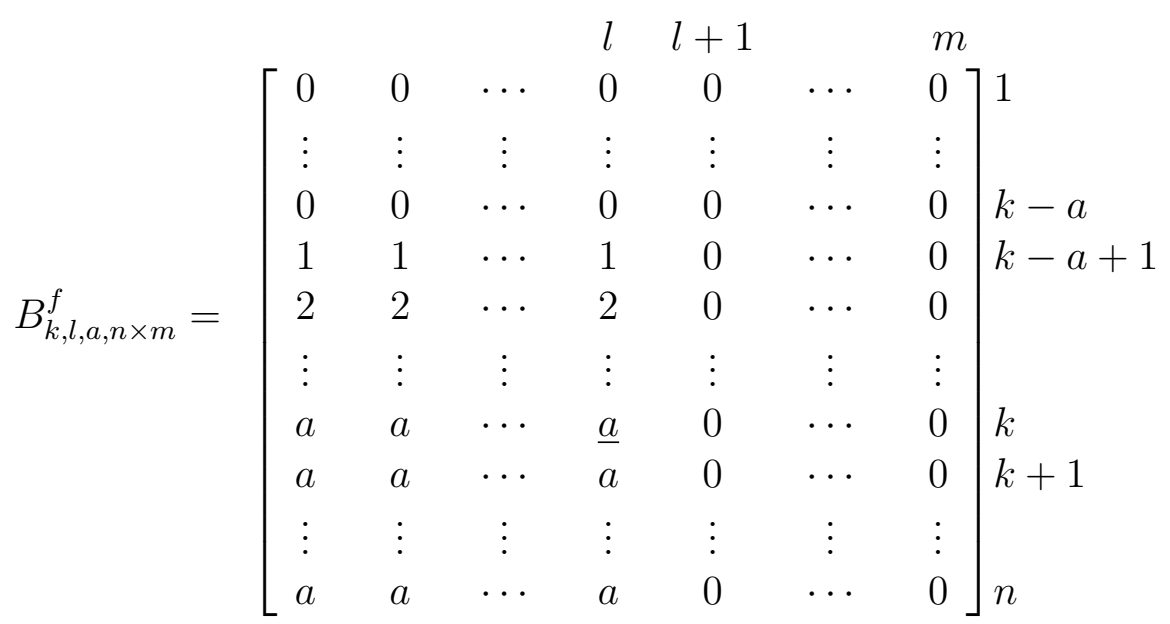


Thus

$$
O^{r}\left(B_{k, l, a, n \times m}^{f}\right)=\left[\begin{array}{ccccccc}
0 & 0 & \ldots & 0 & 0 & \cdots & 0 \\
\vdots & \vdots & \vdots & \vdots & \vdots & \vdots & \vdots \\
0 & 0 & \cdots & 0 & 0 & \cdots & 0 \\
1 & 1 & \cdots & 1 & 0 & \cdots & 0 \\
1 & 1 & \cdots & 1 & 0 & \cdots & 0 \\
\vdots & \vdots & \vdots & \vdots & \vdots & \vdots & \vdots \\
1 & 1 & \cdots & 1 & 0 & \cdots & 0 \\
0 & 0 & \cdots & 0 & 0 & \cdots & 0 \\
\vdots & \vdots & \vdots & \vdots & \vdots & \vdots & \vdots \\
0 & 0 & \cdots & 0 & 0 & \cdots & 0
\end{array}\right] n+1
$$

Hence (see also Remark 8) we obtain the following result.

Proposition 57. Let $1 \leqslant k \leqslant n, 1 \leqslant l \leqslant m$ and $1 \leqslant a \leqslant k$. Then

(a) $B_{k, l, a, n \times m}^{f} \in F_{n}^{m}$.

(b) $f_{B_{k, l, a, n \times m}^{f}}=\left(\begin{array}{ccccccccccc}1 & 2 & \ldots & k-a & k-a+1 & k-a+2 & \ldots & k & k+1 & \ldots & n \\ 0 & 0 & \ldots & 0 & l & l & \ldots & l & 0 & \ldots & 0\end{array}\right)$.

Of course, for $f=\left(\begin{array}{ccccccccccc}1 & 2 & \ldots & r-1 & r & r+1 & \ldots & s & s+1 & \ldots & n \\ 0 & 0 & \ldots & 0 & x & x & \ldots & x & 0 & \ldots & 0\end{array}\right)$, where $1 \leqslant r \leqslant s \leqslant n$ and $1 \leqslant x \leqslant m$, we have $f=f_{B_{s, x, s-r+1, n \times m}^{f}}$. So $M(f)=B_{s, x, s-r+1, n \times m}^{f}$; this fact follows also from the equality $M^{c}(f)=O^{r}\left(B_{s, x, s-r+1, n \times m}^{p}\right)$. Note that $1 \leqslant s-r+1 \leqslant s$.

Now we precisely describe the structure of the poset $\left(\operatorname{Jir}\left(F G_{n}^{m}\right), \leqslant_{M}\right)$. To this purpose we first prove the following technical lemma.

Lemma 58. Let $1 \leqslant k_{1}, k_{2} \leqslant n, 1 \leqslant l_{1}, l_{2} \leqslant m$ and $1 \leqslant a_{1} \leqslant k_{1}, 1 \leqslant a_{2} \leqslant k_{2}$. Then

$$
B_{k_{1}, l_{1}, a_{1}, n \times m}^{f} \leqslant M B_{k_{2}, l_{2}, a_{2}, n \times m}^{f} \quad \text { iff } \quad\left\{\begin{array}{l}
k_{2}-a_{2} \leqslant k_{1}-a_{1} \\
l_{1} \leqslant l_{2} \\
a_{1} \leqslant a_{2}
\end{array} .\right.
$$

$$
B_{k_{1}, l_{1}, a_{1}, n \times m}^{f}=B_{k_{2}, l_{2}, a_{2}, n \times m}^{f} \quad \text { iff } \quad\left(k_{1}, l_{1}, a_{1}\right)=\left(k_{2}, l_{2}, a_{2}\right) .
$$

Proof. Note first that (b) is implied by (a). This fact may be also easily obtained by using the form of matrices $O^{r}\left(B_{k, l, a, n \times m}^{f}\right)$.

(a): Let $C_{1}=B_{k_{1}, l_{1}, a_{1}, n \times m}^{f}$ and $C_{2}=B_{k_{2}, l_{2}, a_{2}, n \times m}^{f}$. Assume that $C_{1} \leqslant{ }_{M} C_{2}$. Then $k_{2}-a_{2}+1 \leqslant k_{1}-a_{1}+1$, because the first non-zero rows in $C_{1}$ and $C_{2}$, respectively, lie in the positions $k_{1}-a_{1}+1$ and $k_{2}-a_{2}+1$, respectively. Next, $a_{1} \leqslant C_{2}\left[k_{1}, l_{1}\right] \leqslant a_{2}$, because all entries of $C_{2}$ are not greater than $a_{2}$. Moreover, $l_{1} \leqslant l_{2}$, because the last non-zero columns in $C_{1}$ and $C_{2}$, respectively, lie in the positions $l_{1}$ and $l_{2}$, respectively. 
Assume that $k_{2}-a_{2} \leqslant k_{1}-a_{1}, l_{1} \leqslant l_{2}$ and $a_{1} \leqslant a_{2}$. In particular, $1 \leqslant a_{1} \leqslant$ $a_{2}-\left(k_{2}-k_{1}\right)$. Then $C_{2}\left[k_{1}, l_{1}\right]=C_{2}\left[k_{1}, l_{2}\right]=$ (because $\left.l_{1} \leqslant l_{2}\right)=C_{2}\left[k_{2}-\left(k_{2}-k_{1}\right), l_{2}\right]=$ $\left\{\begin{array}{ll}a_{2}-\left(k_{2}-k_{1}\right) & \text { if } k_{1} \leqslant k_{2} \\ a_{2} & \text { if } k_{2} \leqslant k_{1}\end{array}\right.$. Thus $a_{1} \leqslant C_{2}\left[k_{1}, l_{1}\right]$, so $C_{1} \leqslant{ }_{M} C_{2}$ by Lemma 39(a).

Theorem 59. $\left(\operatorname{Jir}\left(F G_{n}^{m}\right), \leqslant_{M}\right)$ is isomorphic to a subposet of the poset $\left(\mathbb{N}^{3}, \leqslant_{\text {prod }}\right)$ induced by the set $S_{n, m}=\left\{(x, y, z) \in \mathbb{N}^{3}: 1 \leqslant z \leqslant x \leqslant n\right.$ and $\left.1 \leqslant y \leqslant m\right\}$. Moreover, this isomorphism is given by the function

$$
B_{k, l, a, n \times m}^{f} \longmapsto(n-k+a, l, a) .
$$

Note that $S_{n, m} \subseteq\{1,2, \ldots, n\} \times\{1,2, \ldots, m\} \times\{1,2, \ldots, n\}$.

Proof. Having Lemma 58(b) we can take a function $\Psi: \operatorname{Jir}\left(F G_{n}^{m}\right) \longrightarrow \mathbb{N}^{3}$ such that

$$
\Psi\left(B_{k, l, a, n \times m}^{f}\right)=(n-k+a, l, a) .
$$

By Lemma 58(b) we obtain that $\Psi$ is an embedding of $\left(\operatorname{Jir}\left(F G_{n}^{m}\right), \leqslant_{M}\right)$ into $\left(\mathbb{N}^{3}, \leqslant_{\text {prod }}\right)$. Next, $\Psi\left(\operatorname{Jir}\left(F G_{n}^{m}\right)\right) \subseteq S_{n, m}$, because $1 \leqslant a \leqslant n-k+a=n-(k-a) \leqslant n$. On the other hand, take $(x, y, z) \in S_{n, m}$. Applying inequalities which define the set $S_{n, m}$, it can be shown (simple details are left to the reader) that

$$
1 \leqslant n-x+z \leqslant n, \quad 1 \leqslant y \leqslant m \text { and } 1 \leqslant z \leqslant n-x+z .
$$

Thus we can take the matrix $B_{n-x+z, y, z, n \times m}^{f}$. Of course, $\Psi\left(B_{n-x+z, y, z, n \times m}^{f}\right)=(x, y, z)$. Hence, $S_{n, m} \subseteq \Psi\left(\operatorname{Jir}\left(F G_{n}^{m}\right)\right)$, because $(x, y, z) \in S_{n, m}$ was arbitrarily chosen.

Proposition 60. $\left|\operatorname{Jir}\left(F G_{n}^{m}\right)\right|=\frac{n(n+1)}{2} m$. In particular, $\left|\operatorname{Jir}\left(F G_{n}^{n}\right)\right|=\frac{n^{2}(n+1)}{2}$.

Proof. By Lemma 58(b) we have

$$
\begin{gathered}
\left|\operatorname{Jir}\left(F G_{n}^{m}\right)\right|=\left|\left\{(k, a, l) \in \mathbb{N}^{3}: 1 \leqslant a \leqslant k \leqslant n, 1 \leqslant l \leqslant m\right\}\right|= \\
|\{(k, a): 1 \leqslant a \leqslant k \leqslant n\}| \cdot m=\left(\frac{n^{2}-n}{2}+n\right) \cdot m=\frac{n(n+1)}{2} \cdot m .
\end{gathered}
$$

Since $\left(F G_{n}^{m}, \leqslant_{M}\right)$ is a finite distributive lattice, it is isomorphic to the lattice of all order ideals of $\operatorname{Jir}\left(F G_{n}^{m}\right)$ (see [4] and Subsection 1.1). Hence and by Theorem 59 (see also Subsection 1.1 for the second part of this fact) we obtain

Corollary 61. $\left(F G_{n}^{m}, \leqslant_{M}\right) \simeq\left(O I\left(S_{n, m}\right), \subseteq\right)$. In particular, $\left|F G_{n}^{m}\right|$ is equal to the cardinality of the family of all anti-chains of $\left(S_{n, m}, \leqslant\right.$ prod $)$.

The following fact shows that the poset $\left(S_{n, m}, \leqslant\right.$ prod $)$ introduced in Theorem 59 is self-dual. Its simple proof is left to the reader.

Lemma 62. Let $\varphi: S_{n, m} \longrightarrow S_{n, m}$ be a function such that $\varphi((x, y, z))=(n-z+1, m-$ $y+1, n-x+1)$ for all $(x, y, z) \in S_{n, m}$. Then $\varphi$ is an involutive anti-automorphism of $\left(S_{n, m}, \leqslant_{\text {prod }}\right)$. In particular, $\left(S_{n, m}, \leqslant_{\text {prod }}\right)$ is self-dual. 
By Theorem 59 and Lemma 62 we have that $B_{k, l, a, n \times m}^{f} \longmapsto(n-k+a, l, a) \stackrel{\varphi}{\longmapsto}$ $(n-a+1, m-l+1, n-(n-k+a)+1=(n-a+1, m-l+1, k-a+1)$ and $B_{k, m-l+1, k-a+1, n \times m}^{f} \longmapsto(n-k+(k-a+1), m-l+1, k-a+1)=(n-a+1, m-l+1, k-a+1)$. Thus we have obtained the following fact.

Corollary 63. The function $B_{k, l, a, n \times m}^{f} \longmapsto B_{k, m-l+1, k-a+1, n \times m}^{f}$ is an involutive antiautomorphism of $\left(\operatorname{Jir}\left(F G_{n}^{m}\right), \leqslant_{M}\right)$. In particular, the poset $\left(\operatorname{Jir}\left(F G_{n}^{m}\right), \leqslant_{M}\right)$ is self-dual.

Since the poset $\left(\operatorname{Mir}\left(F G_{n}^{m}\right), \leqslant_{M}\right)$ is isomorphic to the dual of $\left(\operatorname{Jir}\left(F G_{n}^{m}\right), \leqslant_{M}\right)$ (Theorem 15), we obtain by the above corollary that $\left(\operatorname{Mir}\left(F G_{n}^{m}\right), \leqslant_{M}\right)$ is also isomorphic to $\left(\operatorname{Jir}\left(F G_{n}^{m}\right), \leqslant_{M}\right)$, thus also to $\left(S_{n, m}, \leqslant_{M}\right)$. But in the next subsection we describe meetirreducible elements of the lattice $\left(F G_{n}^{m}, \leqslant_{M}\right)$ and consequently, we will be able to give formulas for these isomorphisms.

\subsection{Meet-irreducible elements of the lattice $\left(F G_{n}^{m}, \leqslant_{M}\right)$}

At the beginning of Subsection 3.6 we have shown $\pi_{(k, l)}\left(F G_{n}^{m}\right)=\left\{\mathbf{0}_{n}^{m}[k, l], \mathbf{0}_{n}^{m}[k, l]+\right.$ $\left.1, \ldots, \mathbf{1}_{F_{n}^{m}}[k, l]\right\}$ for all $k=1,2, \ldots, n$ and $l=1,2, \ldots, m$. Thus by Theorem 41 we have that all meet-irreducible elements of the lattice $\left(F G_{n}^{m}, \leqslant M\right)$ are of the form $C_{k, l, a, n \times m}^{F G}$, where $1 \leqslant k \leqslant n, 1 \leqslant l \leqslant m$ and $0=\mathbf{0}_{n}^{m}[k, l] \leqslant a \leqslant \mathbf{1}_{F_{n}^{m}}[k, l]-1=k-1$. These matrices will be denoted by $C_{k, l, a, n \times m}^{f}$ to simplify notation. In other words, we have

$$
\operatorname{Mir}\left(F G_{n}^{m}\right)=\left\{C_{k, l, a, n \times m}^{f}: 1 \leqslant k \leqslant n, 1 \leqslant l \leqslant m \text { and } 0 \leqslant a \leqslant k-1\right\} .
$$

Since $C_{k, l, a, n \times m}^{f}$ is the greatest matrix of $\left(F G_{n}^{m}, \leqslant M\right)$ with the entry $a$ in the position $(k, l)$, we obtain

$$
C_{k, l, a, n \times m}^{f}[i, j]=\left\{\begin{array}{ll}
i & \text { if } 1 \leqslant i \leqslant a \\
i & \text { if } a \leqslant i \text { and } j \leqslant l-1 \\
a & \text { if } a \leqslant i \leqslant k \text { and } j \geqslant l \\
a+i-k & \text { if } i \geqslant k \text { and } j \geqslant l
\end{array},\right.
$$

i.e.,

$$
C_{k, l, a, n \times m}^{f}=\left[\begin{array}{cccccc}
1 & \cdots & 1 & 1 & \cdots & 1 \\
2 & \cdots & 2 & 2 & \cdots & 2 \\
\vdots & \vdots & \vdots & \vdots & \vdots & \vdots \\
a & \cdots & a & a & \cdots & a \\
a+1 & \cdots & a+1 & a & \cdots & a \\
\vdots & \vdots & \vdots & \vdots & \vdots & \vdots \\
k & \cdots & k & \underline{a} & \cdots & a \\
k+1 & \cdots & k+1 & a+1 & \cdots & a+1 \\
\vdots & \vdots & \vdots & \vdots & \vdots & \vdots \\
n & \cdots & n & n-k+a & \cdots & n-k+a
\end{array}\right]_{n}
$$


Thus

$$
O^{r}\left(C_{k, l, a, n \times m}^{f}\right)=\left[\begin{array}{cccccc}
1 & \ldots & 1 & 1 & \ldots & 1 \\
1 & \ldots & 1 & 1 & \ldots & 1 \\
\vdots & \vdots & \vdots & \vdots & \vdots & \vdots \\
1 & \cdots & 1 & 1 & \cdots & 1 \\
1 & \ldots & 1 & 0 & \cdots & 0 \\
\vdots & \vdots & \vdots & \vdots & \vdots & \vdots \\
1 & \ldots & 1 & 0 & \cdots & 0 \\
1 & \ldots & 1 & 1 & \cdots & 1 \\
\vdots & \vdots & \vdots & \vdots & \vdots & \vdots \\
1 & \ldots & 1 & 1 & \cdots & 1
\end{array}\right]_{n}
$$

Hence (see also Remark 8) we obtain the following result.

Proposition 64. Let $1 \leqslant k \leqslant n, 1 \leqslant l \leqslant m$ and $0 \leqslant a \leqslant k-1$. Then

(a) $C_{k, l, a, n \times m}^{f} \in F_{n}^{m}$.

(b) $f_{C_{k, l, a, n \times m}^{f}}=\left(\begin{array}{cccccccccc}1 & 2 & \ldots & a & a+1 & \ldots & k & k+1 & \ldots & n \\ m & m & \ldots & m & l-1 & \ldots & l-1 & m & \ldots & m\end{array}\right)$.

Of course, for $f=\left(\begin{array}{ccccccccccc}1 & 2 & \ldots & r-1 & r & r+1 & \ldots & s & s+1 & \ldots & n \\ m & m & \ldots & m & x & x & \ldots & x & m & \ldots & m\end{array}\right)$, where $1 \leqslant r \leqslant$

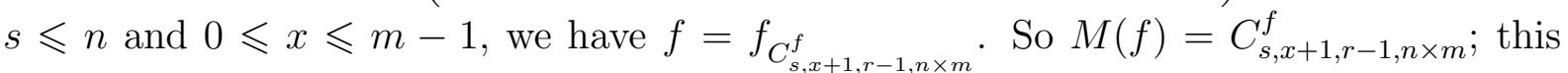
fact follows also from the equality $M^{c}(f)=O^{r}\left(C_{s, x+1, r-1, n \times m}^{p}\right)$. Note that $1 \leqslant x+1 \leqslant m$ and $0 \leqslant r-1 \leqslant s-1$.

The function $A \longmapsto A^{d}$ is an isomorphism between $\left(F G_{n}^{m}, \leqslant_{M}\right)$ and $\left(F G_{n}^{m}, \leqslant_{M}^{d}\right)$ (see Theorem 15). In particular, we have some correspondence between join- and meetirreducible elements of $\left(F G_{n}^{m}, \leqslant_{M}\right)$. The following lemma precisely describes this connection.

Lemma 65. $\left(B_{k, l, a, n \times m}^{f}\right)^{d}=C_{k, m-l+1, k-a, n \times m}^{f}$ and $\left(C_{k, l, a, n \times m}^{f}\right)^{d}=B_{k, m-l+1, k-a, n \times m}^{f}$.

Proof. Recall $A^{d}[i, j]=i-A[i, m-j+1]$, so $A^{d}[i, m-j+1]=i-A[i, j]$. Thus $\left\{A^{d} \in\right.$ $\left.F G_{n}^{m}: A[k, l]=a\right\}=\left\{A^{d} \in F G_{n}^{m}: A^{d}[k, m-l+1]=k-a\right\}=\left\{B \in F G_{n}^{m}: B[k, m-\right.$ $l+1]=k-a\}$ the last equality follows from the fact that $A \longmapsto A^{d}$ is a bijection of $F G_{n}^{m}$. Hence $\left(B_{k, l, a, n \times m}^{f}\right)^{d}=\left(\bigwedge\left\{A \in F G_{n}^{m}: A[k, l]=a\right\}\right)^{d}=\bigvee\left\{A^{d} \in F G_{n}^{m}: A[k, l]=a\right\}$ $=\bigvee\left\{B \in F G_{n}^{m}: B[k, m-l+1]=k-a\right\}=C_{k, m-l+1, k-a, n \times m}^{f}$.

Using the first equality we obtain $\left(B_{k, m-l+1, k-a, n \times m}^{f}\right)^{d}=C_{k, l, a, n \times m}^{f}$, so by Lemma 14 we have $\left(C_{k, l, a, n \times m}^{f}\right)^{d}=\left(\left(B_{k, m-l+1, k-a, n \times m}^{f}\right)^{d}\right)^{d}=B_{k, m-l+1, k-a, n \times m}^{f}$.

Applying Lemma 65, and also results from Subsection 2.4, to Proposition 57 we obtain another proof of Proposition 64. Take a matrix $C_{k, l, a, n \times m}^{f}$. Then $C_{k, l, a, n \times m}^{f}=$ $\left(B_{k, m-l+1, k-a, n \times m}^{f}\right)^{d}$. Hence $C_{k, l, a, n \times m}^{f} \in F_{n}^{m}$ by Lemma $17(\mathrm{~b})$ and Proposition 57. Next, 
$M\left(\mathbf{m}_{n}-f_{C_{k, l, a, n \times m}^{f}}\right)=M\left(f_{C_{k, l, a, n \times m}^{f}}\right)^{d}=B_{k, m-l+1, k-a, n \times m}^{f}$ by Lemma 14 and Lemma 17(a). Thus $\mathbf{m}_{n}-f_{C_{k, l, a, n \times m}^{f}}=\left(\begin{array}{cccccccccc}1 & 2 & \ldots & a=k-(k-a) & a+1 & \ldots & k & k+1 & \ldots & n \\ 0 & 0 & \ldots & 0 & m-l+1 & \ldots & m-l+1 & 0 & \ldots & 0\end{array}\right)$ by Proposition 57. Hence we obtain (b) of Proposition 64.

Take $f=\left(\begin{array}{cccccccccc}1 & 2 & \ldots & r-1 & r & \ldots & s & k+1 & \ldots & n \\ m & m & \ldots & m & y & \ldots & y & m & \ldots & m\end{array}\right)$, where $1 \leqslant r \leqslant s \leqslant n$ and $0 \leqslant$ $y \leqslant m-1$. Then $M\left(\mathbf{m}_{n}-f\right)=B_{s, m-y, s-r+1, n \times m}^{f}$ (see Proposition 57). Hence and by Lemmas 14 and $17(\mathrm{~b}), M(f)=\left(M(f)^{d}\right)^{d}=\left(M\left(\mathbf{m}_{n}-f\right)\right)^{d}=\left(B_{s, m-y, s-r+1, n \times m}^{f}\right)^{d}=$ $C_{s, m-(m-y)+1, s-(s-r+1), n \times m}^{f}=C_{s, y+1, r-1, n \times m}^{f}$.

Observe also that in the case of lattice $\left(F G_{n}^{m}, \leqslant_{M}\right)$ it is sufficient to prove Lemma 39 and Theorems 40, 41 only for one of two kinds of matrices, because then the second case is obtained by Lemma 65 . For example, assume that these results hold for $B_{k, l, a, n \times m}^{f}$, because proofs of second implications are similar.

Lemma 39: $A \leqslant_{M} C_{k, l, a, n \times m}^{f}$ iff $B_{k, m-l+1, k-a, n \times m}^{f}=\left(C_{k, l, a, n \times m}^{f}\right)^{d} \leqslant_{M} A^{d}$ iff (Lemma $39(\mathrm{a})) k-a \leqslant A^{d}[k, m-l+1]=k-A[k, m-(m-l+1)+1]=k-A[k, l]$ iff $A[k, l] \leqslant a$.

Theorem 40: By (a) and Lemma 14 we have $A=\left(A^{d}\right)^{d}=\left(\bigvee\left\{B_{k, l, a, n \times m}^{f}: 1 \leqslant k \leqslant\right.\right.$ $n, 1 \leqslant l \leqslant m$ and $\left.\left.a=A^{d}[k, l] \neq 0\right\}\right)^{d}=\bigwedge\left\{\left(B_{k, l, a, n \times m}^{f}\right)^{d}: 1 \leqslant k \leqslant n, 1 \leqslant l \leqslant m\right.$ and $a=$ $k-A[k, m-l+1] \neq 0\}=\bigwedge\left\{C_{k, m-l+1, k-a, n \times m}^{f}: 1 \leqslant k \leqslant n, 1 \leqslant l \leqslant m\right.$ and $k-a=$ $A[k, m-l+1] \neq k\}=\bigwedge\left\{C_{k^{\prime}, l^{\prime}, a^{\prime}, n \times m}^{f}: 1 \leqslant k^{\prime} \leqslant n, 1 \leqslant l^{\prime} \leqslant m\right.$ and $\left.a^{\prime}=A\left[k^{\prime}, l^{\prime}\right] \neq k^{\prime}\right\}$ (taking $k^{\prime}=k, l^{\prime}=m-l+1$ and $a^{\prime}=k-a$ ).

Theorem 41: $\operatorname{Mir}\left(F G_{n}^{m}\right)=\left\{A^{d}: A \in \operatorname{Jir}\left(F G_{n}^{m}\right)\right\}=\left\{\left(B_{k, l, a, n \times m}^{f}\right)^{d}: 1 \leqslant k \leqslant n, 1 \leqslant\right.$ $l \leqslant m$ and $1 \leqslant a \leqslant k\}=\left\{C_{k, m-l+1, k-a, n \times m}^{f}: 1 \leqslant k \leqslant n, 1 \leqslant l \leqslant m\right.$ and $\left.1 \leqslant a \leqslant k\right\}=$ (taking $k^{\prime}=k, l^{\prime}=m-l+1$ and $\left.a^{\prime}=k-a\right)=\left\{C_{k^{\prime}, l^{\prime}, a^{\prime}, n \times m}^{f}: 1 \leqslant k^{\prime} \leqslant n, 1 \leqslant l^{\prime} \leqslant\right.$ $m$ and $\left.0 \leqslant a^{\prime} \leqslant k-1\right\}$.

Applying Lemma 65 to Lemma 58, Theorem 59 and Proposition 60 we obtain the following three results for meet-irreducible elements of the lattice $\left(F G_{n}^{m}, \leqslant_{M}\right)$.

Lemma 66. Let $1 \leqslant k_{1}, k_{2} \leqslant n, 1 \leqslant l_{1}, l_{2} \leqslant m$ and $0 \leqslant a_{1} \leqslant k_{1}-1,0 \leqslant a_{2} \leqslant k_{2}-1$. Then

$$
C_{k_{1}, l_{1}, a_{1}, n \times m}^{f} \leqslant{ }_{M} C_{k_{2}, l_{2}, a_{2}, n \times m}^{f} \quad \text { iff } \quad\left\{\begin{array}{l}
k_{2}-a_{2} \leqslant k_{1}-a_{1} \\
l_{1} \leqslant l_{2} \\
a_{1} \leqslant a_{2}
\end{array} .\right.
$$

$$
C_{k_{1}, l_{1}, a_{1}, n \times m}^{f}=C_{k_{2}, l_{2}, a_{2}, n \times m}^{f} \quad \text { iff } \quad\left(k_{1}, l_{1}, a_{1}\right)=\left(k_{2}, l_{2}, a_{2}\right) .
$$

Proof. (a): By Theorem 15 and Lemma 65 we obtain $C_{k_{1}, l_{1}, a_{1}, n \times m}^{f} \leqslant_{M} C_{k_{2}, l_{2}, a_{2}, n \times m}^{f}$ iff $\left(C_{k_{2}, l_{2}, a_{2}, n \times m}^{f}\right)^{d} \leqslant{ }_{M}\left(C_{k_{1}, l_{1}, a_{1}, n \times m}^{f}\right)^{d}$ iff $B_{k_{2}, m-l_{2}+1, k_{2}-a_{2}, n \times m}^{f} \leqslant \leqslant_{M} B_{k_{1}, m-l_{1}+1, k_{1}-a_{1}, n \times m}^{f}$. Next, by Lemma 58 (a) we have $B_{k_{2}, m-l_{2}+1, k_{2}-a_{2}, n \times m}^{f} \leqslant M B_{k_{1}, m-l_{1}+1, k_{1}-a_{1}, n \times m}^{f}$ iff

$$
\left\{\begin{array} { l } 
{ k _ { 1 } - ( k _ { 1 } - a _ { 1 } ) \leqslant k _ { 2 } - ( k _ { 2 } - a _ { 2 } ) } \\
{ m - l _ { 2 } + 1 \leqslant m - l _ { 1 } + 1 } \\
{ k _ { 2 } - a _ { 2 } \leqslant k _ { 1 } - a _ { 1 } }
\end{array} \quad \text { iff } \quad \left\{\begin{array}{l}
a_{1} \leqslant a_{2} \\
l_{1} \leqslant l_{2} \\
k_{2}-a_{2} \leqslant k_{1}-a_{1}
\end{array} .\right.\right.
$$


The point (b) follows from (a). Of course, it is also implied by Lemmas 58(b) and 65. Note that this fact may be easily obtained by using the form of matrices $O^{r}\left(C_{k, l, a, n \times m}^{f}\right)$.

Theorem 67. $\left(\operatorname{Mir}\left(F G_{n}^{m}\right), \leqslant_{M}\right)$ is isomorphic to the poset $\left(S_{n, m}, \leqslant_{\text {prod }}\right)$ defined in Theorem59, recall that $\left(S_{n, m}, \leqslant\right.$ prod $)$ is a subposet of the poset $\left(\mathbb{N}^{3}, \leqslant_{\text {prod }}\right)$ such that $S_{n, m}=$ $\left\{(x, y, z) \in \mathbb{N}^{3}: 1 \leqslant z \leqslant x \leqslant n\right.$ and $\left.1 \leqslant y \leqslant m\right\}$. Moreover, this isomorphism is given by the function

$$
C_{k, l, a, n \times m}^{f} \longmapsto(n-k+a+1, l, a+1) .
$$

Proof. It follows from Theorems 15, 59 and Lemmas 62, 65. More precisely, we know that $C_{k, l, a, n \times m}^{f} \longmapsto\left(C_{k, l, a, n \times m}^{f}\right)^{d}=B_{k, m-l+1, k-a, n \times m}^{f}$ is an anti-isomorphism of the poset $\left(\operatorname{Mir}\left(F G_{n}^{m}\right), \leqslant_{M}\right)$ onto the poset $\left(\operatorname{Jir}\left(F G_{n}^{m}\right), \leqslant_{M}\right)$ and $B_{k, l, a, n \times m}^{f} \longmapsto(n-k+a, l, a)$ is an isomorphism of $\left(\operatorname{Jir}\left(F G_{n}^{m}\right), \leqslant_{M}\right)$ onto the poset $\left(S_{n, m}, \leqslant_{\text {prod }}\right)$. Thus their composition $C_{k, l, a, n \times m}^{f} \longmapsto(n-k+(k-a), m-l+1, k-a)=(n-a, m-l+1, k-a)$ is an anti-isomorphism of $\left(\operatorname{Mir}\left(F G_{n}^{m}\right), \leqslant_{M}\right)$ onto $\left(S_{n, m}, \leqslant_{\text {prod }}\right)$. Composing the last function with the anti-automorphism $\varphi$ of $\left(S_{n, m}, \leqslant_{\text {prod }}\right)$ defined in Lemma 62 (recall $\varphi((x, y, z))=$ $(n-z+1, m-y+1, n-x+1))$ we obtain that $C_{k, l, a, n \times m}^{f} \longmapsto(n-(k-a)+1, m-(m-$ $l+1)+1, n-(n-a)+1)=(n-k+a+1, l, a+1)$ is an isomorphism of $\left(\operatorname{Mir}\left(F G_{n}^{m}\right), \leqslant_{M}\right)$ onto $\left(S_{n, m}, \leqslant\right.$ prod $)$.

Proposition 68. $\left|\operatorname{Mir}\left(F G_{n}^{m}\right)\right|=\frac{n(n+1)}{2} m$. In particular, $\left|\operatorname{Mir}\left(F G_{n}^{n}\right)\right|=\frac{n^{2}(n+1)}{2}$.

Proof. It is obtained by Proposition 60, since $\left|\operatorname{Mir}\left(F G_{n}^{m}\right)\right|=\left|\left\{A^{d}: A \in \operatorname{Jir}\left(F G_{n}^{m}\right)\right\}\right|=$ $\left|\operatorname{Jir}\left(F G_{n}^{m}\right)\right|$ (see Theorem 15).

Lemma 66(a) can be also proved in a similar way as Lemma 58(a) (details are left to the reader). Next, Theorem 67 can be easily obtained by Lemma 66(a) (see the proof of Theorem 59). Thus we can obtain alternative proofs of these two results, which do not use the self-duality of lattice $\left(F G_{n}^{m}, \leqslant_{M}\right)$ (i.e., Theorem 15 and Lemma 65). Of course, Proposition 68 can be also shown without using this self-duality in the same way as Proposition 60.

By Theorems 59 and 67 we have that functions $B_{k, l, a, n \times m}^{f} \longmapsto(n-k+a, l, a)$ and $C_{k, l, a-1, n \times m}^{f} \longmapsto(n-k+(a-1)+1, l,(a-1)+1)=(n-k+a, l, a)$, where $1 \leqslant a \leqslant k$, are isomorphisms of $\left(\operatorname{Jir}\left(F G_{n}^{m}\right), \leqslant_{M}\right)$ and $\left(\operatorname{Mir}\left(F G_{n}^{m}\right), \leqslant_{M}\right)$ onto $\left(S_{n, m}, \leqslant_{\text {prod }}\right)$, respectively. Hence obtain the following result.

Corollary 69. Posets $\left(\operatorname{Jir}\left(F G_{n}^{m}\right), \leqslant_{M}\right)$ and $\left(\operatorname{Mir}\left(F G_{n}^{m}\right), \leqslant_{M}\right)$ are isomorphic and this isomorphism is given by the function $B_{k, l, a, n \times m}^{f} \longmapsto C_{k, l, a-1, n \times m}^{f}$ for all $1 \leqslant k \leqslant n$, $1 \leqslant l \leqslant m$ and $1 \leqslant a \leqslant k$.

Take the involutive anti-automorphism $\Psi: B_{k, l, a, n \times m}^{f} \longmapsto B_{k, m-l+1, k-a+1, n \times m}^{f}$ of the poset $\left(\operatorname{Jir}\left(F G_{n}^{m}\right), \leqslant_{M}\right.$ ) (see Corollary 63). By Corollary 69 we have that $\Theta: C_{k, l, a, n \times m}^{f} \longmapsto$ $B_{k, l, a+1, n \times m}^{f}$ is an isomorphism of $\left(\operatorname{Mir}\left(F G_{n}^{m}\right), \leqslant_{M}\right)$ onto $\left(\operatorname{Jir}\left(F G_{n}^{m}\right), \leqslant_{M}\right)$. Hence $\Theta^{-1} \circ \Psi \circ$ $\Theta: C_{k, l, a, n \times m}^{f} \longmapsto B_{k, l, a+1, n \times m}^{f} \longmapsto B_{k, m-l+1, k-(a+1)+1, n \times m}^{f} \longmapsto C_{k, m-l+1, k-(a+1)+1-1, n \times m}^{f}=$ $C_{k, m-l+1, k-a-1, n \times m}^{f}$ is an anti-automorphism of the poset $\left(\operatorname{Mir}\left(F G_{n}^{m}\right), \leqslant_{M}\right)$, which is also an involution. Summarizing, we have shown 
Corollary 70. The function $C_{k, l, a, n \times m}^{f} \longmapsto C_{k, m-l+1, k-a-1, n \times m}^{f}$ is an involutive antiautomorphism of $\left(\operatorname{Mir}\left(F G_{n}^{m}\right), \leqslant_{M}\right)$. In particular, the poset $\left(\operatorname{Mir}\left(F G_{n}^{m}\right), \leqslant_{M}\right)$ is selfdual.

Since $\left(F G_{n}^{m}, \leqslant_{M}\right)$ is a finite distributive lattice, its dual $\left(F G_{n}^{m}, \leqslant_{M}^{d}\right)$ is isomorphic to the lattice of all order filters of $\left(\operatorname{Mir}\left(F G_{n}^{m}\right), \leqslant_{M}\right)$ (see [4] and Subsection 1.1). Hence and by Theorem 67 , since $\left(F G_{n}^{m}, \leqslant_{M}\right)$ is self-dual (see Theorem 15), we obtain

Corollary 71. $\left(F G_{n}^{m}, \leqslant_{M}\right) \simeq\left(O F\left(S_{n, m}\right), \subseteq\right)$.

By this corollary (see also Subsection 1.1) we obtain another proof of the fact that $\left|F G_{n}^{m}\right|$ is equal to the cardinality of the family of all anti-chains of $\left(S_{n, m}, \leqslant\right.$ prod $)$ (see Corollary 61).

Since Theorem 67 can be shown without using the self-duality of lattice $\left(F G_{n}^{m}, \leqslant_{M}\right)$, we can apply this result to obtain another proof of the self-duality of this lattice. More precisely, the lattice $\left(F G_{n}^{m}, \leqslant_{M}\right)$ is isomorphic to $\left(O I\left(S_{n, m}\right), \subseteq\right)$ (see Corollary 61 ), its dual $\left(F G_{n}^{m}, \leqslant_{M}^{d}\right)$ is isomorphic to $\left(O F\left(\operatorname{Mir}\left(F G_{n}^{m}\right), \subseteq\right)\right.$ (see [4] and Subsection 1.1) and $\left(O F\left(\operatorname{Mir}\left(F G_{n}^{m}\right), \subseteq\right) \simeq\left(O F\left(S_{n, m}\right), \subseteq\right)\right.$ (by Theorem 67$)$. Next, the poset $\left(S_{n, m}, \leqslant_{\text {prod }}\right)$ is self-dual (see Lemma 62$)$, so $\left(O I\left(S_{n, m}\right), \subseteq\right) \simeq\left(O I\left(S_{n, m}^{d}\right), \subseteq\right)$, where $S_{n, m}^{d}$ is the dual of $S_{n, m}$. Of course, $O I\left(S_{n, m}^{d}\right)=O F\left(S_{n, m}\right)$.

\subsection{Dedekind-MacNeille completions of posets $\left(F_{n}^{m}, \leqslant_{M}\right)$ and $\left(F(n, m), \leqslant_{F}\right)$}

By Corollary 37 and Propositions 57(a), 64(a) we obtain the following description of Dedekind-Macneille completion of the poset $\left(F_{n}^{m}, \leqslant_{M}\right.$ ) (see also Proposition 10 and Theorem 15).

Theorem 72. $\left(L\left(F_{n}^{m}\right), \subseteq\right) \simeq\left(F G_{n}^{m}, \leqslant_{M}\right)$ (i.e., the Dedekind-MacNeille completion of the poset $\left(F_{n}^{m}, \leqslant_{M}\right)$ is isomorphic to $\left.\left(F G_{n}^{m}, \leqslant_{M}\right)\right)$, in particular, $\left(L\left(F_{n}^{m}\right), \subseteq\right)$ is a finite selfdual distributive lattice. Moreover, $\operatorname{Jir}\left(F_{n}^{m}\right)=\operatorname{Jir}\left(F G_{n}^{m}\right)$ and $\operatorname{Mir}\left(F_{n}^{m}\right)=\operatorname{Mir}\left(F G_{n}^{m}\right)$.

We know that the poset $\left(F(n, m), \leqslant_{F}\right)$, thus also $\left(F_{n}^{m}, \leqslant_{M}\right)$, is self-dual (see Proposition 2), so their Dedekind-MacNeille completions are also self-dual (see Proposition 33). Hence we obtain another proof of the fact that the lattice $\left(F G_{n}^{m}, \leqslant_{M}\right)$ is self-dual (Theorem 15). But this proof does not give a formula for any involutive anti-automorphism of $\left(F G_{n}^{m}, \leqslant M\right)$.

Since the function $f \longmapsto M(f)$ is an isomorphism between posets $\left(F(n, m), \leqslant_{F}\right)$ and $\left(F_{n}^{m}, \leqslant_{M}\right)$, the following properties of the poset $\left(F(n, m), \leqslant_{F}\right)$ are obtained by Theorem 72 and Propositions 57(b), 64(b).

\section{Corollary 73.}

(a) $(L(F(n, m)), \subseteq) \simeq\left(F G_{n}^{m}, \leqslant_{M}\right)$, i.e., the Dedekind-MacNeille completion of the poset $\left(F(n, m), \leqslant_{F}\right)$ is isomorphic to $\left(F G_{n}^{m}, \leqslant_{M}\right)$. In particular, $(L(F(n, m)), \subseteq)$ is a finite self-dual distributive lattice. 
(b) $\operatorname{Jir}(F(n, m))=\left\{f_{B_{k, l, a, n \times m}^{f}}: 1 \leqslant k \leqslant n, 1 \leqslant l \leqslant m, 1 \leqslant a \leqslant k\right\}=$ $\left\{\left(\begin{array}{cccccccccc}1 & 2 & \ldots & r-1 & r & \ldots & s & k+1 & \ldots & n \\ 0 & 0 & \ldots & 0 & y & \ldots & y & 0 & \ldots & 0\end{array}\right) \in F(n, m): 1 \leqslant r \leqslant s \leqslant n, 1 \leqslant y \leqslant m\right\}$.

(c) $\operatorname{Mir}(F(n, m))=\left\{f_{C_{k, l, a, n \times m}^{f}}: 1 \leqslant k \leqslant n, 1 \leqslant l \leqslant m, 0 \leqslant a \leqslant k-1\right\}=$ $\left\{\left(\begin{array}{cccccccccc}1 & 2 & \ldots & r-1 & r & \ldots & s & k+1 & \ldots & n \\ m & m & \ldots & m & y & \ldots & y & m & \ldots & m\end{array}\right) \in F(n, m): 1 \leqslant r \leqslant s \leqslant n, 0 \leqslant y \leqslant\right.$ $m-1\}$.

By Corollary 73, Theorems 59, 67 and Corollaries 63, 69, 70 we obtain the following result which describes the structure of subposets of $\left(F(n, m), \leqslant_{F}\right)$ induced by join- and meet-irreducible elements.

Corollary 74. Let $S_{n, m}=\left\{(x, y, z) \in \mathbb{N}^{3}: 1 \leqslant z \leqslant x \leqslant n, 1 \leqslant y \leqslant m\right\}$. Then

(a) $\left(\operatorname{Jir}(F(n, m)), \leqslant_{F}\right)$ and $\left(\operatorname{Mir}(F(n, m)), \leqslant_{F}\right)$ are isomorphic to $\left(S_{n, m}, \leqslant_{\text {prod }}\right)$. Moreover, these isomorphisms are given by functions

$$
g_{r, s, y}=\left(\begin{array}{cccccccccc}
1 & 2 & \ldots & r-1 & r & \ldots & s & k+1 & \ldots & n \\
0 & 0 & \ldots & 0 & y & \ldots & y & 0 & \ldots & 0
\end{array}\right) \longmapsto(n-r+1, y, s-r+1)
$$

and

$$
h_{r, s, y}=\left(\begin{array}{cccccccccc}
1 & 2 & \ldots & r-1 & r & \ldots & s & k+1 & \ldots & n \\
m & m & \ldots & m & y & \ldots & y & m & \ldots & m
\end{array}\right) \longmapsto(n-s+r, y+1, r) ;
$$

equivalently, by functions

$$
f_{B_{k, l, a, n \times m}^{f}} \longmapsto(n-k+a, l, a) \quad \text { and } \quad f_{C_{k, l, a, n \times m}^{f}} \longmapsto(n-k+a+1, l, a+1) .
$$

(b) The function $g_{r, s, y} \longmapsto h_{s-r+1, s, y-1}$ (equivalently, $f_{B_{k, l, a, n \times m}^{f}} \longmapsto f_{C_{k, l, a-1, n \times m}^{f}}$ ) is an isomorphism of $\left(\operatorname{Jir}(F(n, m)), \leqslant_{F}\right)$ onto $\left(\operatorname{Mir}(F(n, m)), \leqslant_{F}\right)$.

(c) Functions $g_{r, s, y} \longmapsto g_{s-r+1, s, m-y+1}$ and $h_{r, s, y} \longmapsto h_{s-r+1, s, m-y-1}$ (equivalently, functions $f_{B_{k, l, a, n \times m}^{f}} \longmapsto f_{B_{k, m-l+1, k-a+1, n \times m}^{f}}$ and $f_{C_{k, l, a, n \times m}^{f}} \longmapsto f_{C_{k, m-l+1, k-a-1, n \times m}^{f}}$ ) are involutive anti-automorphisms of posets $\left(\operatorname{Jir}(F(n, m)), \leqslant_{F}\right)$ and $\left(\operatorname{Mir}(F(n, m)), \leqslant_{F}\right)$, respectively. In particular, $\left(\operatorname{Jir}(F(n, m)), \leqslant_{F}\right)$ and $\left(\operatorname{Mir}(F(n, m)), \leqslant_{F}\right)$ are selfdual posets.

Proof. Let $\Phi$ denote the isomorphism $f \longmapsto M(f)$ between $\left(F(n, m), \leqslant_{F}\right)$ and $\left(F_{n}^{m}, \leqslant_{M}\right)$. Next, we know

$$
M\left(g_{r, s, y}\right)=B_{s, y, s-r+1, n \times m}^{f} \quad \text { and } \quad M\left(h_{r, s, y}\right)=C_{s, y+1, r-1, n \times m}^{f},
$$

i.e., $g_{r, s, y}=f_{B_{s, y, s-r+1, n \times m}^{f}}$ and $h_{r, s, y}=f_{C_{s, y+1, r-1, n \times m}^{f}}$.

(a): Isomorphisms of $\left(\operatorname{Jir}(F(n, m)), \leqslant_{F}\right)$ and $\left(\operatorname{Mir}(F(n, m)), \leqslant_{F}\right)$ onto $\left(S_{n, m}, \leqslant_{\text {prod }}\right)$ are given by compositions of $\Phi$ with isomorphisms $\Psi_{1}:\left(\operatorname{Jir}\left(F G_{n}^{m}\right), \leqslant_{M}\right) \longrightarrow S_{n, m}$ and $\Psi_{2}:\left(\operatorname{Mir}\left(F G_{n}^{m}\right), \leqslant_{M}\right) \longrightarrow S_{n, m}$ defined in Theorems 59 and 67 . Next,

$\Psi_{1} \circ \Phi\left(g_{r, s, y}\right)=\Psi_{1}\left(B_{s, y, s-r+1, n \times m}^{f}\right)=(n-s+(s-r+1), y, s-r+1)=(n-r+1, y, s-r+1)$ 
and

$\Psi_{2} \circ \Phi\left(h_{r, s, y}\right)=\Psi_{2}\left(C_{s, y+1, r-1, n \times m}^{f}\right)=(n-s+(r-1)+1, y+1,(r-1)+1)=(n-s+r, y+1, r)$.

(b): By Corollary 69, the composition $f_{B_{k, l, a, n \times m}^{f}} \stackrel{\Phi}{\longmapsto} B_{k, l, a, n \times m}^{f} \longmapsto C_{k, l, a-1, n \times m}^{f} \stackrel{\Phi^{-1}}{\longmapsto}$ $f_{C_{k, l, a-1, n \times m}^{f}}$ is an isomorphism of $\left(\operatorname{Jir}(F(n, m)), \leqslant_{F}\right)$ onto $\left(\operatorname{Mir}(F(n, m)), \leqslant_{F}\right)$. Hence we have $g_{r, s, y}=f_{B_{s, y, s-r+1, n \times m}^{f}} \longmapsto f_{C_{s, y, s-r, n \times m}^{f}}=h_{s-r+1, s, y-1}$.

Of course, this point can be also obtained by (a), because $\Phi^{-1} \circ \Psi_{2}^{-1} \circ \Psi_{1} \circ \Phi$ is an isomorphism of $\left(\operatorname{Jir}(F(n, m)), \leqslant_{F}\right)$ onto $\left(\operatorname{Mir}(F(n, m)), \leqslant_{F}\right)$ and $\Psi_{1} \circ \Phi\left(g_{r, s, y}\right)=\Psi_{2} \circ$ $\Phi\left(h_{s-r+1, s, y-1}\right)$.

(c): By Corollary 63 we obtain that the composition $f_{B_{k, l, a, n \times m}^{f}} \stackrel{\Phi}{\longmapsto} B_{k, l, a, n \times m}^{f} \longmapsto$ $B_{k, m-l+1, k-a+1, n \times m}^{f} \stackrel{\Phi^{-1}}{\longmapsto} f_{B_{k, m-l+1, k-a+1, n \times m}^{f}}$ is an involutive anti-automorphism of the poset $\left(\operatorname{Jir}(F(n, m)), \leqslant_{F}\right)$. Hence we have $g_{r, s, y}=f_{B_{s, y, s-r+1, n \times m}^{f}} \longmapsto f_{B_{s, m-y+1, s-(s-r+1)+1, n \times m}^{f}}=$ $f_{B_{s, m-y+1, r, n \times m}^{f}}=g_{s-r+1, s, m-y+1}$.

Next, by Corollary 70 we obtain that the composition $f_{C_{k, l, a, n \times m}^{f}} \stackrel{\Phi}{\longmapsto} C_{k, l, a, n \times m}^{f} \longmapsto$ $C_{k, m-l+1, k-a-1, n \times m}^{f} \stackrel{\Phi^{-1}}{\longrightarrow} f_{C_{k, m-l+1, k-a-1, n \times m}^{f}}$ is an involutive anti-automorphism of the poset $\left(\operatorname{Mir}(F(n, m)), \leqslant_{F}\right)$. Hence we have $h_{r, s, y}=f_{C_{s, y+1, r-1, n \times m}^{f}} \longmapsto f_{C_{s, m-(y+1)+1, s-(r-1)-1, n \times m}^{f}}=$ $f_{C_{s, m-y, s-r, n \times m}^{f}}=h_{s-r+1, s, m-y-1}$.

Of course, self-duality of $\left(\operatorname{Jir}(F(n, m)), \leqslant_{F}\right)$ and $\left(\operatorname{Mir}(F(n, m)), \leqslant_{F}\right)$ is also obtained by (b) and Proposition 2. But by Corollaries 63 and 70 we have formulas for their antiautomorphisms.

\subsection{Remarks on posets $\left(T_{n}^{m}, \leqslant_{M}\right),\left(T(n, m), \leqslant_{F}\right)$ and the lattice $\left(T G_{n}^{m}, \leqslant_{M}\right)$}

Since $T G_{n}^{1}=T_{n}^{1}=\left\{\mathbf{0}_{T_{n}^{1}}\right\}=\left\{\left[\begin{array}{lllll}1 & 2 & 3 & \ldots & n\end{array}\right]^{T}\right\}$ and $T(n, m)=\left\{\mathbf{1}_{n}\right\}$ are one-element sets, we assume here that $m \geqslant 2$. Note that one-element sets form trivial lattices which have no join- and meet-irreducible elements.

Recall that the function $\Psi: A \longmapsto \downarrow A$ is an isomorphism of the lattice $\left(T G_{n}^{m}, \leqslant_{M}\right)$ onto the lattice $\left(F G_{n}^{m-1}, \leqslant_{M}\right)$ and its inverse $\Psi^{-1}$ is given by the function $B \longmapsto \uparrow B$. Moreover, $\Psi\left(T_{n}^{m}\right)=F_{n}^{m-1}$. Thus all results from the last three subsections concerning the poset $\left(F_{n}^{m}, \leqslant_{M}\right)$ and the lattice $\left(F G_{n}^{m}, \leqslant_{M}\right)$ can be translated for $\left(T_{n}^{m}, \leqslant_{M}\right)$ and $\left(T G_{n}^{m}, \leqslant M\right)$.

We start with a description of join- and meet-irreducible elements of the lattice $\left(T G_{n}^{m}, \leqslant_{M}\right)$. By the definition of the set $T G_{n}^{m}$ (see Definition 9 ) and properties of the bottom $\mathbf{0}_{T_{n}^{m}}$ and the top element $\mathbf{1}_{F_{n}^{m}}$ of the lattice $\left(T G_{n}^{m}, \leqslant_{M}\right)$ (see the last part of Subsection 2.3) we obtain $\pi_{(k, l)}\left(T G_{n}^{m}\right)=\left\{\mathbf{0}_{T_{n}^{m}}[k, l], \mathbf{0}_{T_{n}^{m}}[k, l]+1, \ldots, \mathbf{1}_{F_{n}^{m}}[k, l]\right\}$ for all $k=1,2, \ldots, n$ and $l=1,2, \ldots, m$. Hence and by Theorem 41 we have that all join- and meet-irreducible elements are of the form $B_{k, l, a, n \times m}^{T G}$ and $C_{k, l, b, n \times m}^{T G}$, respectively, where $1 \leqslant k \leqslant n, 1 \leqslant l \leqslant m$ and $\mathbf{0}_{T_{n}^{m}}[k, l]+1 \leqslant a \leqslant \mathbf{1}_{F_{n}^{m}}[k, l]=k, \mathbf{0}_{T_{n}^{m}}[k, l] \leqslant b \leqslant \mathbf{1}_{F_{n}^{m}}[k, l]-1=k-1$. These matrices will be denoted by $B_{k, l, a, n \times m}^{t}$ and $C_{k, l, b, n \times m}^{t}$, respectively, to simplify notation. Since 
$\mathbf{0}_{T_{n}^{m}}[k, 1]=k$, there are no integers $a$ and $b$ such that $k+1 \leqslant a \leqslant k$ and $k \leqslant b \leqslant k-1$. Therefore we can assume that $l \geqslant 2$. Summarizing, we obtain the following facts (recall that $\mathbf{0}_{T_{n}^{m}}[k, l]=0$ for $\left.l \geqslant 2\right)$.

$$
\begin{gathered}
\operatorname{Jir}\left(T G_{n}^{m}\right)=\left\{B_{k, l, a, n \times m}^{t}: 1 \leqslant k \leqslant n, 2 \leqslant l \leqslant m \text { and } 1 \leqslant a \leqslant k\right\}, \\
\operatorname{Mir}\left(T G_{n}^{m}\right)=\left\{C_{k, l, a, n \times m}^{t}: 1 \leqslant k \leqslant n, 2 \leqslant l \leqslant m \text { and } 0 \leqslant a \leqslant k-1\right\} .
\end{gathered}
$$

From the other hand, all join- and meet-irreducible elements of $\left(T G_{n}^{m}, \leqslant M\right)$ are of the form $\uparrow B_{k, l, a, n \times m-1}^{f}$ and $\uparrow C_{k, l, b, n \times m-1}^{f}$, respectively, for $1 \leqslant k \leqslant n, 1 \leqslant l \leqslant m-1$ and $1 \leqslant a \leqslant k, 0 \leqslant b \leqslant k-1$. By Definition 38 we obtain the following relationships between these two kinds of descriptions of irreducible elements.

For every $1 \leqslant k \leqslant n, 2 \leqslant l \leqslant m$ and $1 \leqslant a \leqslant k, 0 \leqslant b \leqslant k-1$ we have that $\downarrow B_{k, l, a, n \times m}^{t}=\downarrow \bigwedge\left\{A \in T G_{n}^{m}: A[k, l]=a\right\}=\bigwedge\left\{\downarrow A \in F G_{n}^{m-1}: A \in T G_{n}^{m}\right.$ and $A[k, l]=$ $a\}=\bigwedge\left\{B \in F G_{n}^{m-1}: B[k, l-1]=a\right\}=B_{k, l-1, a, n \times m-1}^{f}$ and similarly, $\downarrow C_{k, l, b, n \times m}^{t}=$ $C_{k, l-1, b, n \times m-1}^{f}$. Hence

$$
B_{k, l, a, n \times m}^{t}=\uparrow B_{k, l-1, a, n \times m-1}^{f} \quad \text { and } \quad C_{k, l, a, n \times m}^{t}=\uparrow C_{k, l-1, a, n \times m-1}^{f} .
$$

By these equalities and Lemmas 58(b), 66(b) we obtain that matrices $B_{k, l, a, n \times m}^{t}$ and $C_{k, l, a, n \times m}^{t}$ are uniquely determined by the triple $(k, l, a)$. Next, the following facts hold.

(1) The function $B_{k, l, a, n \times m}^{t} \longmapsto B_{k, l-1, a, n \times m-1}^{f}$ is an isomorphism of $\left(\operatorname{Jir}\left(T G_{n}^{m}\right), \leqslant_{M}\right)$ onto $\left(\operatorname{Jir}\left(F G_{n}^{m-1}\right), \leqslant_{M}\right)$ and its inverse is $B_{k, l, a, n \times m-1}^{f} \longmapsto B_{k, l+1, a, n \times m}^{t}$.

(2) The function $C_{k, l, a, n \times m}^{t} \longmapsto C_{k, l-1, a, n \times m-1}^{f}$ is an isomorphism of $\left(\operatorname{Mir}\left(T G_{n}^{m}\right), \leqslant_{M}\right)$ onto $\left(\operatorname{Mir}\left(F G_{n}^{m-1}\right), \leqslant_{M}\right)$ and its inverse is $C_{k, l, a, n \times m-1}^{f} \longmapsto C_{k, l+1, a, n \times m}^{t}$.

Thus Theorems 59 and 67 imply that compositions

$$
B_{k, l, a, n \times m}^{t} \longmapsto B_{k, l-1, a, n \times m-1}^{f} \longmapsto(n-k+a, a, l-1)
$$

and

$$
C_{k, l, a, n \times m}^{t} \longmapsto C_{k, l-1, a, n \times m-1}^{f} \longmapsto(n-k+a+1, a+1, l-1)
$$

are isomorphisms of $\left(\operatorname{Jir}\left(T G_{n}^{m}\right), \leqslant_{M}\right)$ and $\left(\operatorname{Mir}\left(T G_{n}^{m}\right), \leqslant_{M}\right)$ onto $\left(S_{n, m-1}, \leqslant_{\text {prod }}\right)$, respectively. Hence posets $\left(\operatorname{Jir}\left(T G_{n}^{m}\right), \leqslant_{M}\right)$ and $\left(\operatorname{Mir}\left(T G_{n}^{m}\right), \leqslant_{M}\right)$ are isomorphic and this isomorphism is given by the function $B_{k, l, a, n \times m}^{t} \longmapsto C_{k, l, a-1, n \times m}^{t}$ (of course, it follows also from Corollary 69).

By Propositions 60 and 68 we have $\mid \operatorname{Jir}\left(T G_{n}^{m}|=| \operatorname{Mir}\left(T G_{n}^{m} \mid=\frac{n(n+1)}{2}(m-1)\right.\right.$, in particular, $\mid \operatorname{Jir}\left(T G_{n}^{n}|=| \operatorname{Mir}\left(T G_{n}^{n} \mid=\frac{n\left(n^{2}-1\right)}{2}\right.\right.$.

By properties (1), (2) and Corollaries 63, 70 we obtain that the following compositions

$$
\begin{aligned}
B_{k, l, a, n \times m}^{t} & \longmapsto B_{k, l-1, a, n \times m-1}^{f} \longmapsto B_{k, m-1-(l-1)+1, k-a+1, n \times m-1}^{f}= \\
B_{k, m-l+1, k-a+1, n \times m-1}^{f} & \longmapsto B_{k, m-l+2, k-a+1, n \times m}^{t}
\end{aligned}
$$


and

$$
\begin{aligned}
& C_{k, l, a, n \times m}^{t} \longmapsto C_{k, l-1, a, n \times m-1}^{f} \longmapsto C_{k, m-1-(l-1)+1, k-a-1, n \times m-1}^{f}= \\
& C_{k, m-l+1, k-a-1, n \times m-1}^{f} \longmapsto C_{k, m-l+2, k-a-1, n \times m}^{t}
\end{aligned}
$$

are involutive anti-automorphisms of posets $\left(\operatorname{Jir}\left(T G_{n}^{m}\right), \leqslant_{M}\right)$ and $\left(\operatorname{Mir}\left(T G_{n}^{m}\right), \leqslant_{M}\right)$, respectively. In particular, $\left(\operatorname{Jir}\left(T G_{n}^{m}\right), \leqslant_{M}\right)$ and $\left(\operatorname{Mir}\left(T G_{n}^{m}\right), \leqslant_{M}\right)$ are self-dual posets.

By Corollaries 61 and 71 we have $\left(T G_{n}^{m}, \leqslant_{M}\right) \simeq\left(O I\left(S_{n, m-1}\right), \subseteq\right)$ and $\left(T G_{n}^{m}, \leqslant_{M}\right) \simeq$ $\left(O F\left(S_{n, m-1}\right), \subseteq\right)$.

Since $\Psi_{\left\lceil T_{n}^{m}\right.}$ is an isomorphism between posets $\left(T_{n}^{m}, \leqslant_{F}\right)$ and $\left(F_{n}^{m-1}, \leqslant_{F}\right)$, Theorem 72 implies that the Dedekind-MacNeille completion $\left(L\left(T_{n}^{m}\right), \subseteq\right)$ of $\left(T_{n}^{m}, \leqslant_{M}\right)$ is isomorphic to the lattice $\left(F G_{n}^{m-1}, \leqslant_{M}\right)$, so also to the lattice $\left(T G_{n}^{m}, \leqslant_{M}\right)$. In particular, it is a finite selfdual distributive lattice. Moreover, $\operatorname{Jir}\left(T_{n}^{m}\right)=\Psi^{-1}\left(\operatorname{Jir}\left(F_{n}^{m-1}\right)\right)=\Psi^{-1}\left(\operatorname{Jir}\left(F G_{n}^{m-1}\right)\right)=$ $\operatorname{Jir}\left(T G_{n}^{m}\right)$ and $\operatorname{Mir}\left(T_{n}^{m}\right)=\Psi^{-1}\left(\operatorname{Mir}\left(F_{n}^{m-1}\right)\right)=\Psi^{-1}\left(\operatorname{Mir}\left(F G_{n}^{m-1}\right)\right)=\operatorname{Mir}\left(T G_{n}^{m}\right)$.

Since the function $f \longmapsto M(f)$ is an isomorphism between posets $\left(T(n, m), \leqslant_{F}\right)$ and $\left(T_{n}^{m}, \leqslant_{M}\right)$, we obtain by the above fact that the Dedekind-MacNeille completion $(L(T(n, m)), \subseteq)$ of the poset $\left(T(n, m), \leqslant_{F}\right)$ is isomorphic to the lattice $\left(F G_{n}^{m-1}, \leqslant_{M}\right)$, so also to the lattice $\left(T G_{n}^{m}, \leqslant_{M}\right)$. In particular, it is a finite self-dual distributive lattice.

To describe join- and meet-irreducible elements of the poset $\left(T(n, m), \leqslant_{F}\right)$ observe that $M\left(f+\mathbf{1}_{n}\right)=\uparrow M(f)$ for all $f \in F G_{n}^{m-1}$. Hence, for all $1 \leqslant k \leqslant n, 2 \leqslant l \leqslant m$ and $1 \leqslant a \leqslant k$, we have $M\left(f_{B_{k, l, a, n \times m}^{t}}\right)=B_{k, l, a, n \times m}^{t}=\uparrow B_{k, l-1, a, n \times m-1}^{f}=\uparrow M\left(f_{B_{k, l-1, a, n \times m-1}^{f}}\right)=$ $M\left(f_{B_{k, l-1, a, n \times m-1}^{f}}+\mathbf{1}_{n}\right)$, so by Theorem 6 and Proposition 57 we obtain

$$
f_{B_{k, l, a, n \times m}^{t}}=f_{B_{k, l-1, a, n \times m-1}^{f}}+\mathbf{1}_{n}=\left(\begin{array}{ccccccccccc}
1 & 2 & \ldots & k-a & k-a+1 & k-a+2 & \ldots & k & k+1 & \ldots & n \\
1 & 1 & \ldots & 1 & l & l & \ldots & l & 1 & \ldots & 1
\end{array}\right) .
$$

Similarly, for all $1 \leqslant k \leqslant n, 2 \leqslant l \leqslant m$ and $0 \leqslant a \leqslant k-1$ we have the equality $M\left(f_{C_{k, l, a, n \times m}^{t}}\right)=M\left(f_{C_{k, l-1, a, n \times m-1}^{f}}+\mathbf{1}_{n}\right)$, so by Theorem 6 and Proposition 64 we obtain

$$
f_{C_{k, l, a, n \times m}^{t}}=f_{C_{k, l-1, a, n \times m-1}^{f}}+\mathbf{1}_{n}=\left(\begin{array}{ccccccccccc}
1 & 2 & \ldots & a & a+1 & a+2 & \ldots & k & k+1 & \ldots & n \\
m & m & \ldots & m & l-1 & l-1 & \ldots & l-1 & m & \ldots & m
\end{array}\right) .
$$

By these equalities and Corollary 73(b),(c) we obtain

$$
\begin{gathered}
\operatorname{Jir}(T(n, m))=\left\{f_{B_{k, l, a, n \times m}^{t}}: 1 \leqslant k \leqslant n, 2 \leqslant l \leqslant m, 1 \leqslant a \leqslant k\right\}= \\
\left\{f_{\left.B_{k, l, a, n \times m-1}^{f}+\mathbf{1}_{n}: 1 \leqslant k \leqslant n, 1 \leqslant l \leqslant m-1,1 \leqslant a \leqslant k\right\}=}=1 \leqslant n \leqslant n, 2 \leqslant y \leqslant m\right\} . \\
\left\{\left(\begin{array}{cccccccccc}
1 & 2 & \ldots & r-1 & r & \ldots & s & k+1 & \ldots & n \\
1 & 1 & \ldots & 1 & y & \ldots & y & 1 & \ldots & 1
\end{array}\right) \in T(n, m): 1 \leqslant r \leqslant s \leqslant n\right.
\end{gathered}
$$

and

$$
\begin{aligned}
& \operatorname{Mir}(T(n, m))=\left\{f_{C_{k, l, a, n \times m}^{t}}: 1 \leqslant k \leqslant n, 2 \leqslant l \leqslant m, 0 \leqslant a \leqslant k-1\right\}= \\
& \left\{f_{C_{k, l, a, n \times m-1}^{f}}+\mathbf{1}_{n}: 1 \leqslant k \leqslant n, 1 \leqslant l \leqslant m-1,0 \leqslant a \leqslant k-1\right\}= \\
& \left\{\left(\begin{array}{cccccccccc}
1 & 2 & \ldots & r-1 & r & \ldots & s & k+1 & \ldots & n \\
m & m & \ldots & m & y & \ldots & y & m & \ldots & m
\end{array}\right) \in T(n, m): 1 \leqslant r \leqslant s \leqslant n, 1 \leqslant y \leqslant m-1\right\} .
\end{aligned}
$$


Recall (see the end of Subsection 1.2) that we have the isomorphism $\Theta:(F(n, m-$ $\left.1), \leqslant_{F}\right) \longrightarrow\left(T(n, m), \leqslant_{F}\right)$ such that $\Theta(f)=f+\mathbf{1}_{n}$ for all $f \in F(n, m-1)$. Next, its inverse $\Theta^{-1}$ is given by the formula $\Theta^{-1}(g)=g-\mathbf{1}_{n}$ for all $g \in T(n, m)$. Applying the isomorphism $\Theta^{-1}$, Corollary 73 and the fact $\left(F G_{n}^{m-1}, \leqslant_{M}\right) \simeq\left(T G_{n}^{m}, \leqslant_{M}\right)$ we also obtain the above results concerning the poset $\left(T(n, m), \leqslant_{F}\right)$ (simple details are left to the reader).

By facts (1), (2) and Corollary 74 we obtain that posets $\left(\operatorname{Jir}(T(n, m)), \leqslant_{F}\right)$ and $\left(\operatorname{Mir}(T(n, m)), \leqslant_{F}\right)$ are isomorphic to the poset $\left(S_{n, m-1}, \leqslant_{\text {prod }}\right)$ and these isomorphisms are given by functions

$$
f_{B_{k, l, a, n \times m}^{t}} \longmapsto(n-k+a, a, l-1) \quad \text { and } \quad f_{C_{k, l, a, n \times m}^{t}} \longmapsto(n-k+a+1, a+1, l-1) .
$$

In particular, $\left(\operatorname{Jir}(T(n, m)), \leqslant_{F}\right)$ and $\left(\operatorname{Mir}(T(n, m)), \leqslant_{F}\right)$ are isomorphic and this isomorphism is given by the function $f_{B_{k, l, a, n \times m}^{t}} \longmapsto f_{C_{k, l, a-1, n \times m}^{t}}$. Next, functions

$$
f_{B_{k, l, a, n \times m}^{t}} \longmapsto f_{B_{k, m-l+2, k-a+1, n \times m}^{t}} \text { and } \quad f_{C_{k, l, a, n \times m}^{t}} \longmapsto f_{C_{k, m-l+2, k-a-1, n \times m}^{t}}
$$

are involutive anti-automorphisms of $\left(\operatorname{Jir}(T(n, m)), \leqslant_{F}\right)$ and $\left(\operatorname{Mir}(T(n, m)), \leqslant_{F}\right)$, respectively, so these posets are self-dual. Observe that applying Corollary 74, the isomorphism $\Theta:\left(F(n, m-1), \leqslant_{F}\right) \longrightarrow\left(T(n, m), \leqslant_{F}\right)$ and its inverse $\Theta^{-1}$ we obtain that all functions given in this paragraph can be expressed directly in terms of elements of $\operatorname{Jir}(T(n, m))$ and $\operatorname{Mir}(T(n, m))$ (without using isomorphisms $B_{k, l, a, n \times m}^{t} \longmapsto f_{B_{k, l, a, n \times m}^{t}}$ and $C_{k, l, a, n \times m}^{t} \longmapsto f_{C_{k, l, a, n \times m}^{t}}$ between posets $\left(\operatorname{Jir}\left(T G_{n}^{m}\right), \leqslant_{M}\right),\left(\operatorname{Jir}(T(n, m)), \leqslant_{F}\right)$ and $\left(\operatorname{Mir}\left(T G_{n}^{m}\right), \leqslant_{M}\right),\left(\operatorname{Mir}(T(n, m)), \leqslant_{F}\right)$, respectively), but technical details are left to the readers.

\subsection{Join-irreducible elements of the lattice $\left(I G_{n}^{m}, \leqslant_{M}\right)$}

Since $I G_{n}^{m}=\emptyset$ for $m<n$, we assume in this subsection that $n \leqslant m$. Then by the definition of the set $I G_{n}^{m}$ (see Definition 9) and properties of the bottom $\Delta_{n}^{m}$ and the top element $\nabla_{n}^{m}$ of the lattice $\left(I G_{n}^{m}, \leqslant_{M}\right)$ (see the last part of Subsection 2.3) we obtain that $\pi_{(k, l)}\left(I G_{n}^{m}\right)=$ $\left\{\Delta_{n}^{m}[k, l], \Delta_{n}^{m}[k, l]+1, \ldots, \nabla_{n}^{m}[k, l]\right\}$ for all $k=1,2, \ldots, n$ and $l=1,2, \ldots, m$. Hence and by Theorem 41 we have that all join-irreducible elements of the lattice $\left(I G_{n}^{m}, \leqslant M\right)$ are of the form $B_{k, l, a, n \times m}^{I G}$, where $1 \leqslant k \leqslant n, 1 \leqslant l \leqslant m$ and $\max \{1, k-l+2\}=\max \{0, k-$ $l+1\}+1=\Delta_{n}^{m}[k, l]+1 \leqslant a \leqslant \nabla_{n}^{m}[k, l]=\min \{k, m+1-l\} \leqslant \min \{n, m\}=n$. These matrices will be denoted by $B_{k, l, a, n \times m}^{i}$ to simplify notation. Since $\Delta_{n}^{m}[k, 1]=\nabla_{n}^{m}[k, 1]=k$, there is no integer $a$ such that $\Delta_{n}^{m}[k, 1]+1 \leqslant a \leqslant \nabla_{n}^{m}[k, 1]$. Therefore we can assume that $l \geqslant 2$. Summarizing, we obtain

$$
\operatorname{Jir}\left(I G_{n}^{m}\right)=
$$

$\left\{B_{k, l, a, n \times m}^{i}: 1 \leqslant k \leqslant n, 2 \leqslant l \leqslant m\right.$ and $\left.\max \{0, k-l+1\}+1 \leqslant a \leqslant \min \{k, m+1-l\}\right\}$.

For the lattice $\left(S G_{n}^{n}, \leqslant_{M}\right)$ we have that $\Delta_{n}^{n}[n, l]=\nabla_{n}^{n}[n, l]=n-l+1$, so in this case we can additionally assume that $k \leqslant n-1$. Thus we obtain $\operatorname{Jir}\left(S G_{n}^{n}\right)=\left\{B_{k, l, a, n \times n}^{i}: 1 \leqslant\right.$ $k \leqslant n-1,2 \leqslant l \leqslant n$ and $\max \{0, k-l+1\}+1 \leqslant a \leqslant \min \{k, n+1-l\}\}$. 
Since $B_{k, l, a, n \times m}^{i}$ is the least matrix of $\left(I G_{n}^{m}, \leqslant_{M}\right)$ with the entry $a$ in the position $(k, l)$, we obtain that $B_{k, l, a, n \times m}^{i}$ looks as follows (note that $l+a-1 \leqslant m$ ):

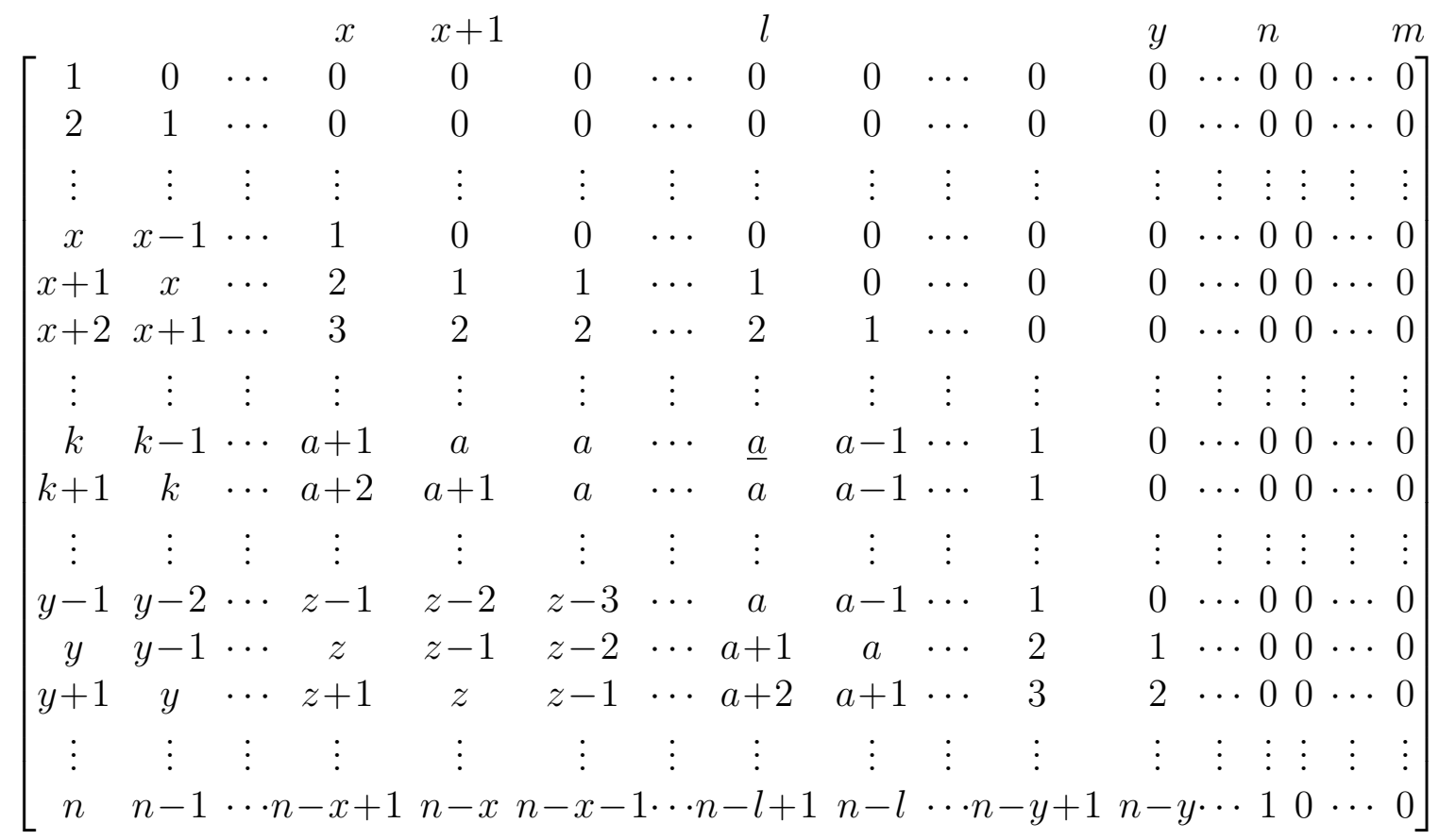

where $x=k-a, y=k+(l-(k-a))=l+a$ and $z=y-x+1=l+2 a-k+1$.

Of course, some rows and columns do not appear in the above matrix for some values of $k, l$ and $a$. For instance, for $a=k$ or $n \leqslant l+a-1$.

Thus the matrix $O^{r}\left(B_{k, l, a, n \times m}^{i}\right)$ equals

$$
\left[\begin{array}{ccccccccccccccccccc}
1 & 0 & \cdots & 0 & 0 & 0 & 0 & \cdots & 0 & 0 & 0 & \cdots & 0 & 0 & \cdots & 0 & 0 & \cdots & 0 \\
1 & 1 & \cdots & 0 & 0 & 0 & 0 & \cdots & 0 & 0 & 0 & \cdots & 0 & 0 & \cdots & 0 & 0 & \cdots & 0 \\
\vdots & \vdots & \vdots & \vdots & \vdots & \vdots & \vdots & \vdots & \vdots & \vdots & \vdots & \vdots & \vdots & \vdots & \vdots & \vdots & \vdots & \vdots & \vdots \\
1 & 1 & \cdots & 1 & 0 & 0 & 0 & \cdots & 0 & 0 & 0 & \cdots & 0 & 0 & \cdots & 0 & 0 & \cdots & 0 \\
1 & 1 & \cdots & 1 & 1 & 1 & 1 & \cdots & 1 & 1 & 0 & \cdots & 0 & 0 & \cdots & 0 & 0 & \cdots & 0 \\
1 & 1 & \cdots & 1 & 1 & 1 & 1 & \cdots & 1 & 1 & 1 & \cdots & 0 & 0 & \cdots & 0 & 0 & \cdots & 0 \\
\vdots & \vdots & \vdots & \vdots & \vdots & \vdots & \vdots & \vdots & \vdots & \vdots & \vdots & \vdots & \vdots & \vdots & \vdots & \vdots & \vdots & \vdots & \vdots \\
1 & 1 & \cdots & 1 & 1 & 1 & 1 & \cdots & 1 & 1 & 1 & \cdots & 1 & 0 & \cdots & 0 & 0 & \cdots & 0 \\
1 & 1 & \cdots & 1 & 1 & 0 & 0 & \cdots & 0 & 0 & 0 & \cdots & 0 & 0 & \cdots & 0 & 0 & \cdots & 0 \\
1 & 1 & \cdots & 1 & 1 & 1 & 0 & \cdots & 0 & 0 & 0 & \cdots & 0 & 0 & \cdots & 0 & 0 & \cdots & 0 \\
\vdots & \vdots & \vdots & \vdots & \vdots & \vdots & \vdots & \vdots & \vdots & \vdots & \vdots & \vdots & \vdots & \vdots & \vdots & \vdots & \vdots & \vdots & \vdots \\
1 & 1 & \cdots & 1 & 1 & 1 & 1 & \cdots & 1 & 0 & 0 & \cdots & 0 & 0 & \cdots & 0 & 0 & \cdots & 0 \\
1 & 1 & \cdots & 1 & 1 & 1 & 1 & \cdots & 1 & 1 & 1 & \cdots & 1 & 1 & \cdots & 0 & 0 & \cdots & 0 \\
1 & 1 & \cdots & 1 & 1 & 1 & 1 & \cdots & 1 & 1 & 1 & \cdots & 1 & 1 & \cdots & 0 & 0 & \cdots & 0 \\
\vdots & \vdots & \vdots & \vdots & \vdots & \vdots & \vdots & \vdots & \vdots & \vdots & \vdots & \vdots & \vdots & \vdots & \vdots & \vdots & \vdots & \vdots & \vdots \\
1 & 1 & \cdots & 1 & 1 & 1 & 1 & \cdots & 1 & 1 & 1 & \cdots & 1 & 1 & \cdots & 1 & 0 & \cdots & 0
\end{array}\right] x
$$


where $x=k-a$ and $y=k+(l-(k-a))=l+a$.

Hence (see also Remark 8) we obtain the following result.

Proposition 75. Let $1 \leqslant k \leqslant n, 2 \leqslant l \leqslant m$ and $\max \{0, k-l+1\}+1 \leqslant a \leqslant$ $\min \{k, m+1-l\}$. Then

(a) $B_{k, l, a, n \times m}^{i} \in I_{n}^{m}$.

(b) $f_{B_{k, l, a, n \times m}^{i}}$ is such that

(b.1) if $l+a-1 \leqslant n$, then $f_{B_{k, l, a, n \times m}^{i}}=\left(\begin{array}{ccccccccc}1 & 2 & \ldots & k-a & k-a+1 & k-a+2 & \ldots & k \\ 1 & 2 & \ldots & k-a & l & l+1 & \ldots & l+a-1 \\ k+1 & k+2 & \ldots & l+a-1 & l+a & l+a+1 & \ldots & n \\ k-a+1 & k-a+2 & \ldots & l-1 & l+a & l+a+1 & \ldots & n\end{array}\right)$, (note that the inequality $\max \{0, k-l+1\}+1 \leqslant a$ implies $k-a \leqslant l-2$ and $k \leqslant l+a-2)$,

in particular, if $l+a-1=n$, then $f_{B_{k, l, a, n \times m}^{i}}=\left(\begin{array}{ccccc}1 & 2 & \ldots & k-a & k-a+1 \\ 1 & 2 & \ldots & k-a & l\end{array}\right.$ $\left.\begin{array}{cccccccc}k-a+2 & \ldots & k & k+1 & k+2 & \ldots & l+a-2 & l+a-1=n \\ l+1 & \ldots & l+a-1 & k-a+1 & k-a+2 & \ldots & l-2 & l-1\end{array}\right)$,

(b.2) if $n+1 \leqslant l+a-1 \leqslant m$, then $f_{B_{k, l, a, n \times m}^{i}}=\left(\begin{array}{ccccccc}1 & 2 & \ldots & k-a & k-a+1 & k-a+2 & \ldots \\ 1 & 2 & \ldots & k-a & l & l+1 & \ldots\end{array}\right.$ $\left.\begin{array}{ccccc}k & k+1 & k+2 & \cdots & (k-a)+(n-k)=n-a\end{array}\right)$.

Take $f=\left(\begin{array}{ccccccccccccccc}1 & 2 & \ldots & r-1 & r & r+1 & \ldots & s & s+1 & s+2 & \ldots & s+y-r & y+s-r+1 & \ldots & n \\ 1 & 2 & \ldots & r-1 & y & y+1 & \ldots & y+s-r & r & r+1 & \ldots & y-1 & y+s-r+1 & \ldots & n\end{array}\right)$ for some $1 \leqslant r \leqslant s \leqslant n$ and $r+1 \leqslant y \leqslant m-s+r$; of course, if $s+y-r \geqslant$ $n$ and $s<n$, then the tail of $f$ equals $\left.\begin{array}{ccccc}\ldots & s+1 & s+2 & \ldots & n \\ \ldots & r & r+1 & \ldots & r+(n-s)-1\end{array}\right)$. Then $f=$ $f_{B_{s, y, s-r+1, n \times m}^{i}}$. So $M(f)=B_{s, y, s-r+1, n \times m}^{i}$; this fact follows also from the equality $M^{c}(f)=$ $O^{r}\left(B_{s, y, s-r+1, n \times m}^{i}\right)$. Note that $\max \{0, s-y+1\}+1 \leqslant s-r+1 \leqslant \min \{s, m+1-y\}$.

If $n=m$, then the case (b.2) of Proposition 75 does not hold. Thus by this proposition we obtain the following fact describing bijections corresponding to join-irreducible elements of the lattice $\left(S G_{n}^{n}, \leqslant_{M}\right)$.

Corollary 76. Let $1 \leqslant k \leqslant n-1,2 \leqslant l \leqslant n$ and $\max \{0, k-l+1\}+1 \leqslant a \leqslant$ $\min \{k, n+1-l\}$. Then

(a) $B_{k, l, a, n \times n}^{i} \in I_{n}^{n}$.

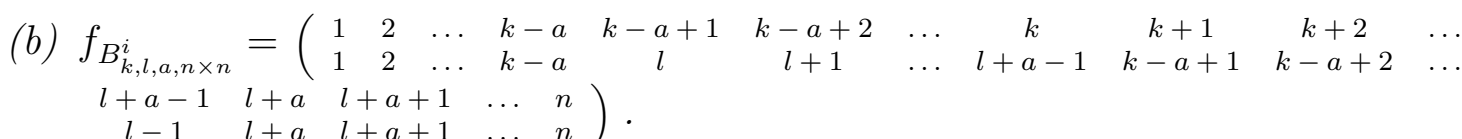

Now we precisely describe the structure of the poset $\left(\operatorname{Jir}\left(I G_{n}^{m}\right), \leqslant_{M}\right)$. To this purpose we first prove the following technical lemma. 
Lemma 77. Let $1 \leqslant k_{1}, k_{2} \leqslant n, 2 \leqslant l_{1}, l_{2} \leqslant m$ and $\max \left\{0, k_{1}-l_{1}+1\right\}+1 \leqslant a_{1} \leqslant$ $\min \left\{k_{1}, m+1-l_{1}\right\}, \max \left\{0, k_{2}-l_{2}+1\right\}+1 \leqslant a_{2} \leqslant \min \left\{k_{2}, m+1-l_{2}\right\}$. Then

$$
B_{k_{1}, l_{1}, a_{1}, n \times m}^{i} \leqslant \leqslant_{M} B_{k_{2}, l_{2}, a_{2}, n \times m}^{i} \quad \text { iff } \quad\left\{\begin{array}{l}
k_{1}-a_{1} \geqslant k_{2}-a_{2} \\
l_{1}+a_{1} \leqslant l_{2}+a_{2} \\
a_{1} \leqslant a_{2} \\
a_{1}+l_{1}-k_{1} \leqslant a_{2}+l_{2}-k_{2}
\end{array}\right.
$$

$$
B_{k_{1}, l_{1}, a_{1}, n \times m}^{i}=B_{k_{2}, l_{2}, a_{2}, n \times m}^{i} \quad \text { iff } \quad\left(k_{1}, l_{1}, a_{1}\right)=\left(k_{2}, l_{2}, a_{2}\right) .
$$

Proof. Note first that (b) is implied by (a). This fact may be also obtained by using the form of the matrix $O^{r}\left(B_{k, l, a, n \times m}^{i}\right)$, but this is a quite complicated method in this case.

(a): Let $C_{1}=B_{k_{1}, l_{1}, a_{1}, n \times m}^{i}$ and $C_{2}=B_{k_{2}, l_{2}, a_{2}, n \times m}^{i}$. By Lemma 39(a) we have the equivalence

$$
C_{1} \leqslant{ }_{M} C_{2} \text { iff } a_{1} \leqslant C_{2}\left[k_{1}, l_{1}\right] .
$$

If $k_{1} \leqslant k_{2}-a_{2}$ or $l_{1} \leqslant k_{2}-a_{2}+1$ or $l_{1} \geqslant l_{2}+a_{2}$ or $k_{1} \geqslant l_{2}+a_{2}-1$, then $C_{2}\left[k_{1}, l_{1}\right]=$ $\max \left\{0, k_{1}-l_{1}+1\right\}<\max \left\{0, k_{1}-l_{1}+1\right\}+1 \leqslant a_{1}$. Thus we have the following four cases:

(a.1): $k_{2}-a_{2}+1 \leqslant k_{1} \leqslant k_{2}$ and $k_{2}-a_{2}+2 \leqslant l_{1} \leqslant l_{2}$. Then $C_{2}\left[k_{1}, l_{1}\right]=C_{2}\left[k_{1}, l_{2}\right]=$ $C_{2}\left[k_{2}-\left(k_{2}-k_{1}\right), l_{2}\right]=a_{2}-\left(k_{2}-k_{1}\right)$, so $C_{1} \leqslant{ }_{M} C_{2}$ iff $a_{1} \leqslant a_{2}-\left(k_{2}-k_{1}\right)$ iff $k_{2}-a_{2} \leqslant k_{1}-a_{1}$. All other inequalities (from the right-hand side of first equivalence) are implied by the proved inequality $k_{2}-a_{2} \leqslant k_{1}-a_{1}$ and our assumptions in this case. More precisely, $k_{1} \leqslant k_{2}$ and $k_{2}-a_{2} \leqslant k_{1}-a_{1}$ imply $a_{1} \leqslant a_{2}$; hence $l_{1}+a_{1} \leqslant l_{2}+a_{2}$, because $l_{1} \leqslant l_{2}$; finally, $l_{1} \leqslant l_{2}$ and $a_{1}-k_{1} \leqslant a_{2}-k_{2}$ imply $a_{1}+l_{1}-k_{1} \leqslant a_{2}+l_{2}-k_{2}$. The similar situation will hold in the next three cases, therefore we will not explicit formulate it later.

(a.2) $k_{2}-a_{2}+1 \leqslant k_{1} \leqslant k_{2}$ and $l_{2} \leqslant l_{1} \leqslant l_{2}+a_{2}-1$. If $k_{1}-l_{1}<k_{2}-a_{2}+1-l_{2}$ (i.e., the position $\left(k_{1}, l_{1}\right)$ lies to the right from the line passing through positions $\left(k_{2}-a_{2}+1, l_{2}\right)$ and $\left.\left(k_{2}, l_{2}+a_{2}-1\right)\right)$, then $C_{2}\left[k_{1}, l_{1}\right]=0$. Thus we can assume that $k_{1}-l_{1} \geqslant k_{2}-a_{2}+1-l_{2}$ (i.e., the position $\left(k_{1}, l_{1}\right)$ lies to the left from the line passing through positions $\left(k_{2}-a_{2}+1, l_{2}\right)$ and $\left.\left(k_{2}, l_{2}+a_{2}-1\right)\right)$. Then $C_{2}\left[k_{1}, l_{1}\right]=C_{2}\left[k_{2}-\left(k_{2}-k_{1}\right), l_{2}+\left(l_{1}-l_{2}\right)\right]=a_{2}-\left(k_{2}-k_{1}\right)-\left(l_{1}-l_{2}\right)$. Hence $C_{1} \leqslant{ }_{M} C_{2}$ iff $a_{1} \leqslant a_{2}-\left(k_{2}-k_{1}\right)-\left(l_{1}-l_{2}\right)$ iff $a_{1}+l_{1}-k_{1} \leqslant a_{2}+l_{2}-k_{2}$. (a.3): $k_{2} \leqslant k_{1} \leqslant l_{2}+a_{2}-2$ and $k_{2}-a_{2}+2 \leqslant l_{1} \leqslant l_{2}$. If $k_{1}-l_{1} \geqslant a_{2}-1$ (i.e., the position $\left(k_{1}, l_{1}\right)$ lies to the left from the line passing through positions $\left(k_{2}, k_{2}-a_{2}+1\right)$ and $\left.\left(l_{2}+a_{2}-1, l_{2}\right)\right)$, then $C_{2}\left[k_{1}, l_{1}\right]=k_{1}-l_{1}+1<k_{1}-l_{1}+2 \leqslant a_{1}$. Thus we can assume that $k_{1}-l_{1}<a_{2}-1$ (i.e., the position $\left(k_{1}, l_{1}\right)$ lies to the right from the line passing through positions $\left(k_{2}, k_{2}-a_{2}+1\right)$ and $\left.\left(l_{2}+a_{2}-1, l_{2}\right)\right)$. Then $C_{2}\left[k_{1}, l_{1}\right]=a_{2}$, so $C_{1} \leqslant{ }_{M} C_{2}$ iff $a_{1} \leqslant a_{2}$.

(a.4): $k_{2} \leqslant k_{1} \leqslant l_{2}+a_{2}-2$ and $l_{2} \leqslant l_{1} \leqslant l_{2}+a_{2}-1$. Then $C_{2}\left[k_{1}, l_{1}\right]=C_{2}\left[k_{1}, l_{2}+\left(l_{1}-l_{2}\right)\right]=$ $a_{2}-\left(l_{1}-l_{2}\right)$, so $C_{1} \leqslant{ }_{M} C_{2}$ iff $a_{1} \leqslant a_{2}-\left(l_{1}-l_{2}\right)$ iff $l_{1}+a_{1} \leqslant l_{2}+a_{2}$.

Theorem 78. $\left(\operatorname{Jir}\left(I G_{n}^{m}\right), \leqslant_{M}\right)$ is isomorphic to a subposet of the poset $\left(\mathbb{N}^{4}, \leqslant_{\text {prod }}\right)$ induced by the set $R_{n, m}=\left\{(x, y, z, t) \in \mathbb{N}^{4}: 1 \leqslant x \leqslant n, 2 \leqslant y \leqslant m, 1 \leqslant z \leqslant \min \{x, y-1\}, n+\right.$ 
$1 \leqslant x+y-z$ and $t=x+y-z\}$. Moreover, this isomorphism is given by the function

$$
B_{k, l, a, n \times m}^{i} \longmapsto(n-k+a, l+a-1, a, a+l-k+n-1) .
$$

Note that $R_{n, m} \subseteq\{1,2, \ldots, n\} \times\{2,3, \ldots, m\} \times\{1,2, \ldots, n\} \times\{n+1, n+2, \ldots, m+n-1\}$ (recall, $n \leqslant m)$.

Proof. Having Lemma 77(b) we can take a function $\Psi: \operatorname{Jir}\left(I G_{n}^{m}\right) \longrightarrow \mathbb{N}^{4}$ such that

$$
\Psi\left(B_{k, l, a, n \times m}^{i}\right)=(n-k+a, l+a-1, a, a+l-k)+(0,0,0, n-1) .
$$

Lemma $77\left(\right.$ a) implies that $\Psi$ is an embedding of $\left(\operatorname{Jir}\left(I G_{n}^{m}\right), \leqslant_{M}\right)$ into $\left(\mathbb{N}^{4}, \leqslant_{\text {prod }}\right)$. Next, for each $B_{k, l, a, n \times m}^{i}$ we have $1 \leqslant a \leqslant n-k+a \leqslant n$ and $2 \leqslant a+1=2+a-1 \leqslant l+a-1 \leqslant m$, because $a \leqslant \min \{k, m+1-l\}$. Moreover, $(n-k+a)+(l+a-1)-a=a+l-k+n-1$ and $a+l-k \geqslant 2$, because $a \geqslant \max \{1, k-l+2\}$. Note also $a+l-k \leqslant l \leqslant m$. By all these facts we obtain that $\Psi\left(\operatorname{Jir}\left(I G_{n}^{m}\right)\right) \subseteq R_{n, m}$. On the other hand, take $(x, y, z, t) \in R_{n, m}$. Applying inequalities which define the set $R_{n, m}$, it can be shown (technical details are left to the reader) that

$$
1 \leqslant n-x+z \leqslant n, \quad 2 \leqslant y-z+1 \leqslant m
$$

and

$$
\max \{0,(n-x+z)-(y-z+1)+1\}+1 \leqslant z \leqslant \min \{n-x+z, m+1-(y-z+1)\}
$$

(for example, the inequality $n+1 \leqslant x+y-z$ implies $n-x+z-y+z-1+1+1 \leqslant z$, so $(n-x+z)-(y-z+1)+2 \leqslant z)$. Thus we can take the matrix $B_{n-x+z, y-z+1, z, n \times m}^{i}$. Of course, $\Psi\left(B_{n-x+z, y-z+1, z, n \times m}^{i}\right)=(x, y, z, x+y-z)=(x, y, z, t)$. Hence $R_{n, m} \subseteq \Psi\left(\operatorname{Jir}\left(I G_{n}^{m}\right)\right)$, because $(x, y, z, t) \in R_{n, m}$ was arbitrarily chosen.

Proposition 79. $\left|\operatorname{Jir}\left(I G_{n}^{m}\right)\right|=\frac{3 m n(m-n)+(n-1) n(n+1)}{6}$.

In particular, $\left|\operatorname{Jir}\left(S G_{n}^{n}\right)\right|=\frac{(n-1) n(n+1)}{6}$.

Proof. By Lemma 77(b) we have

$$
\left|\operatorname{Jir}\left(I G_{n}^{m}\right)\right|=
$$

$\mid\left\{(k, l, a) \in \mathbb{N}^{3}: 1 \leqslant k \leqslant n, 2 \leqslant l \leqslant m, k-l+2 \leqslant a \leqslant k\right.$ and $\left.1 \leqslant a \leqslant m+1-l\right\} \mid=$ $\mid\left\{(k, l, a) \in \mathbb{N}^{3}: 1 \leqslant k \leqslant n, 2 \leqslant l \leqslant m, k-a+2 \leqslant l, 1 \leqslant k-a+1\right.$ and $\left.1 \leqslant a \leqslant m+1-l\right\} \mid$.

Hence, setting $x=k-a+1, y=l$ and $z=a$, we obtain

$$
\left|\operatorname{Jir}\left(I G_{n}^{m}\right)\right|=|S|,
$$

where $S=\left\{(x, y, z) \in \mathbb{N}^{3}: 1 \leqslant x \leqslant n, 2 \leqslant y \leqslant m, x+1 \leqslant y\right.$ and $\left.1 \leqslant z \leqslant m-y+1\right\}$.

Next, $|S|=\sum_{y=2}^{n}(y-1)(m-y+1)+\sum_{y=n+1}^{m} n(m-y+1)=\sum_{y=2}^{n}(m-(y-$ 1)) $(y-1)+n \sum_{y=n+1}^{m}(m-y+1)=\sum_{y=2}^{n}\left(m(y-1)-(y-1)^{2}\right)+n \sum_{y=n+1}^{m}(m-y+$ 1) $=m \sum_{y=2}^{n}(y-1)-\sum_{y=2}^{n}(y-1)^{2}+n \sum_{t=1}^{m-n} t=m \frac{(n-1) n}{2}-\frac{(n-1)(n-1+1)(2(n-1)+1)}{6}+$ $n \frac{(m-n)(m-n+1)}{2}=\frac{3 m n(m-n)+(n-1) n(n+1)}{6}$. 
Since $\left(I G_{n}^{m}, \leqslant_{M}\right)$ is a finite distributive lattice, it is isomorphic to the lattice of all order ideals of $\operatorname{Jir}\left(I G_{n}^{m}\right)$ (see [4] and Subsection 1.1). Hence and by Theorem 78 (see also Subsection 1.1 for the second part of this fact) we obtain

Corollary 80. $\left(I G_{n}^{m}, \leqslant_{M}\right) \simeq\left(O I\left(R_{n, m}\right), \subseteq\right)$. In particular, $\left|I G_{n}^{m}\right|$ is equal to the cardinality of the family of all anti-chains of $\left(R_{n, m}, \leqslant\right.$ prod $)$.

\subsection{Meet-irreducible elements of the lattice $\left(I G_{n}^{m}, \leqslant_{M}\right)$}

Similarly as in the previous subsection we assume here that $n \leqslant m$. Recall also that at the beginning of the previous subsection we have shown $\pi_{(k, l)}\left(I G_{n}^{m}\right)=\left\{\Delta_{n}^{m}[k, l], \Delta_{n}^{m}[k, l]+\right.$ $\left.1, \ldots, \nabla_{n}^{m}[k, l]\right\}$ for all $k=1,2, \ldots, n$ and $l=1,2, \ldots, m$. Thus by Theorem 41 we have that all meet-irreducible elements of the lattice $\left(I G_{n}^{m}, \leqslant_{M}\right)$ are of the form $C_{k, l, a, n \times m}^{I G}$, where $1 \leqslant k \leqslant n, 1 \leqslant l \leqslant m$ and $\max \{0, k-l+1\}=\Delta_{n}^{m}[k, l] \leqslant a \leqslant \nabla_{n}^{m}[k, l]-1=$ $\min \{k, m+1-l\}-1=\min \{k-1, m-l\}$. These matrices will be denoted by $C_{k, l, a, n \times m}^{i}$ to simplify notation. Of course, the inequality $\max \{0, k-l+1\} \leqslant \min \{k, m+1-l\}-1$ does not hold for $l=1$, because $k \leqslant n \leqslant m$ (see also the beginning of the previous subsection). Thus we can assume that $l \geqslant 2$. Summarizing, we obtain

$$
\operatorname{Mir}\left(I G_{n}^{m}\right)=
$$

$\left\{C_{k, l, a, n \times m}^{i}: 1 \leqslant k \leqslant n, 2 \leqslant l \leqslant m\right.$ and $\left.\max \{0, k-l+1\} \leqslant a \leqslant \min \{k, m+1-l\}-1\right\}$.

For the lattice $S G_{n}^{n}$, similarly as in the case of join-irreducible elements (see the beginning of the previous subsection), we can additionally assume that $k \leqslant n-1$. Thus $\operatorname{Mir}\left(S G_{n}^{n}\right)=$ $\left\{C_{k, l, a, n \times n}^{i}: 1 \leqslant k \leqslant n-1,2 \leqslant l \leqslant m\right.$ and $\left.\max \{0, k-l+1\} \leqslant a \leqslant \min \{k, m+1-l\}-1\right\}$.

The function $A \longmapsto A^{t d}$ is an involutive anti-automorphism of the lattice $\left(I G_{n}^{m} \leqslant M\right)$ (see Corollary 22). In particular, we have some correspondence between join- and meetirreducible elements of $I G_{n}^{m}$. The following lemma precisely describes this connection.

Lemma 81. $\left(B_{k, l, a, n \times m}^{i}\right)^{t d}=C_{k, m-l+2, k-a, n \times m}^{i}$ and $\left(C_{k, l, a, n \times m}^{i}\right)^{t d}=B_{k, m-l+2, k-a, n \times m}^{i}$.

Proof. Take $k, l, a$ such that $1 \leqslant k \leqslant n, 2 \leqslant l \leqslant m$ and $\max \{0, k-l+1\}+1 \leqslant$ $a \leqslant \min \{k, m+1-l\}$. Recall that if $j \geqslant 2$, then $A^{t d}[i, j]=i-A[i, m-j+2]$. So $A^{t d}[k, m-l+2]=k-A[k, m-(m-l+2)+2]=k-A[k, l]$. Thus $\left\{A^{t d} \in I G_{n}^{m}: A[k, l]=\right.$ $a\}=\left\{A^{t d} \in I G_{n}^{m}: A^{t d}[k, m-l+2]=k-a\right\}=\left\{B \in I G_{n}^{m}: B[k, m-l+2]=k-a\right\}$, the second equality follows from the fact that $A \longmapsto A^{t d}$ is a bijection of $I G_{n}^{m}$. Hence

$$
\begin{gathered}
\left(B_{k, l, a, n \times m}^{i}\right)^{t d}=\left(\bigwedge\left\{A \in I G_{n}^{m}: A[k, l]=a\right\}\right)^{t d}=\bigvee\left\{A^{t d} \in I G_{n}^{m}: A[k, l]=a\right\} \\
=\bigvee\left\{B \in I G_{n}^{m}: B[k, m-l+2]=k-a\right\}=C_{k, m-l+2, k-a, n \times m}^{i} .
\end{gathered}
$$

The inequalities $\max \{1, k-l+2\} \leqslant a \leqslant \min \{k, m+1-l\}$ imply $\max \{0, k-(m-l+$ $2)+1\}=\max \{0, k+l-m-1\} \leqslant k-a \leqslant \min \{k-1, l-2\}=\min \{k-1, m-(m-l+2)\}$.

Take $k, l, a$ such that $1 \leqslant k \leqslant n, 2 \leqslant l \leqslant m$ and $\max \{0, k-l+1\} \leqslant a \leqslant \min \{k-$ $1, m-l\}$. Then it is easy to see that $\max \{1, k-(m-l+2)+2\}=\max \{1, k+$ 
$l-m\} \leqslant k-a \leqslant \min \{k, l-1\}=\min \{k, m+1-(m-l+2)\}$. Thus we can take the matrix $B_{k, m-l+2, k-a, n \times m}^{i}$. Then $\left(B_{k, m-l+2, k-a, n \times m}^{i}\right)^{t d}=C_{k, l, a, n \times m}^{i}$, so $\left(C_{k, l, a, n \times m}^{i}\right)^{t d}=$ $\left(\left(B_{k, m-l+2, k-a, n \times m}^{i}\right)^{t d}\right)^{t d}=B_{k, m-l+2, k-a, n \times m}^{i}$ by Lemma 20.

By Lemma 81 we obtain that in the case of the lattice $\left(I G_{n}^{m}, \leqslant_{M}\right)$ it is sufficient to show Lemma 39 and Theorems 40, 41 only for one of two kinds of matrices. Proofs of these equivalences are similar to proofs of analogous results for the lattice $\left(F G_{n}^{m}, \leqslant_{M}\right)$ (see Subsection 3.7). For example, assume that these results hold for $B_{k, l, a, n \times m}^{i}$, because proofs of second implications are similar.

Lemma 39: $A \leqslant_{M} C_{k, l, a, n \times m}^{i}$ iff $B_{k, m-l+2, k-a, n \times m}^{i}=\left(C_{k, l, a, n \times m}^{i}\right)^{t d} \leqslant_{M} A^{t d}$ iff (Lemma $39(\mathrm{a})) k-a \leqslant A^{t d}[k, m-l+2]=k-A[k, m-(m-l+2)+2]=k-A[k, l]$ (because $l \geqslant 2)$ iff $A[k, l] \leqslant a$.

Theorem 40: By (a) and Lemma 20 we have $A=\left(A^{t d}\right)^{t d}=\left(\bigvee\left\{B_{k, l, a, n \times m}^{i}: 1 \leqslant\right.\right.$ $k \leqslant n, 2 \leqslant l \leqslant m$ and $\left.\left.a=A^{t d}[k, l] \neq 0\right\}\right)^{t d}=\bigwedge\left\{\left(B_{k, l, a, n \times m}^{i}\right)^{t d}: 1 \leqslant k \leqslant n, 2 \leqslant l \leqslant\right.$ $m$ and $a=k-A[k, m-l+2] \neq 0\}=\bigwedge\left\{C_{k, m-l+2, k-a, n \times m}^{i}: 1 \leqslant k \leqslant n, 2 \leqslant l \leqslant m\right.$ and $k-$ $a=A[k, m-l+2] \neq k\}=\bigwedge\left\{C_{k^{\prime}, l^{\prime}, a^{\prime}, n \times m}^{i}: 1 \leqslant k^{\prime} \leqslant n, 2 \leqslant l^{\prime} \leqslant m\right.$ and $\left.a^{\prime}=A\left[k^{\prime}, l^{\prime}\right] \neq k^{\prime}\right\}$ (taking $k^{\prime}=k, l^{\prime}=m-l+2$ and $a^{\prime}=k-a$ ).

Theorem 41: $\operatorname{Mir}\left(I G_{n}^{m}\right)=\left\{A^{t d}: A \in \operatorname{Jir}\left(I G_{n}^{m}\right)\right\}=\left\{\left(B_{k, l, a, n \times m}^{i}\right)^{i d}: 1 \leqslant k \leqslant n, 2 \leqslant\right.$ $l \leqslant m$ and $\max \{0, k-l+1\}+1 \leqslant a \leqslant \min \{k, m+1-l\}\}=\left\{C_{k, m-l+2, k-a, n \times m}^{i}: 1 \leqslant\right.$ $k \leqslant n, 2 \leqslant l \leqslant m$ and $\max \{1, k-l+2\} \leqslant a \leqslant \min \{k, m+1-l\}\}=$ (taking $k^{\prime}=k, l^{\prime}=m-l+2$ and $\left.a^{\prime}=k-a\right)=\left\{C_{k^{\prime}, l^{\prime}, a^{\prime}, n \times m}^{i}: 1 \leqslant k^{\prime} \leqslant n, 2 \leqslant l^{\prime} \leqslant\right.$ $m$ and $\left.\max \left\{0, k^{\prime}-l^{\prime}+1\right\} \leqslant a^{\prime} \leqslant \min \left\{k^{\prime}-1, m-l^{\prime}\right\}\right\}$.

By Lemmas 24, 81 and Proposition 75 we obtain that $C_{k, l, a, n \times m}^{i} \in I_{n}^{m}$. Next,

$$
\begin{gathered}
C_{k, l, a, n \times m}^{i}=\left(B_{k, m-l+2, k-a, n \times m}^{i}\right)^{t d}= \\
\left(M\left(f_{B_{k, m-l+2, k-a, n \times m}^{i}}\right)\right)^{t d}=M\left(\mathbf{m}_{n}-f_{B_{k, m-l+2, k-a, n \times m}^{i}}+\mathbf{1}_{n}\right) .
\end{gathered}
$$

Hence (see also Remark 8)

$$
f_{C_{k, l, a, n \times m}^{i}}=\mathbf{m}_{n}-f_{B_{k, m-l+2, k-a, n \times m}^{i}}+\mathbf{1}_{n} .
$$

This equality and Proposition 75(b) may be used to described injective functions corresponding to matrices $C_{k, l, a, n \times m}^{i}$. But we want also to describe the structure of matrices $C_{k, l, a, n \times m}^{i}$. Of course, matrices $C_{k, l, a, n \times m}^{i}$ and $C_{k, l, a, n \times m}^{p}$ have similar forms. Thus we can use results given in Subsection 3.4 for $C_{k, l, a, n \times m}^{p}$ (see the proof of Proposition 47) to sketch the structure of matrices $C_{k, l, a, n \times m}^{i}$. In particular, we obtain formulas for functions $f_{C_{k, l, a, n \times m}^{i} \times}$.

By the inequality $\max \{0, k-l+1\} \leqslant a$ we have that $k-a \leqslant l-1$, so $x=$ $a+\min \{l-1, k-a\}=k$, where $x$ is the parameter in the first matrix given in Subsection 3.4. This matrix describes the first $k+2$ rows of $C_{k, l, a, n \times m}^{i}$. Moreover, these first $k+2$ rows correspond to the following function (recall that $l \leqslant m-a$ ):

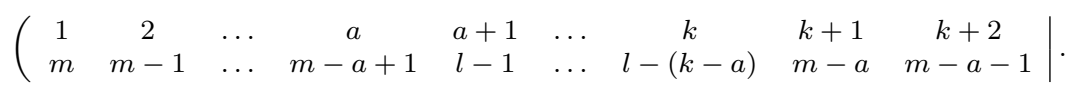


Assume that $m-a+1-l \geqslant n-k$ or equivalently $m-a-(n-k) \geqslant l-1$ (i.e., the distance between the $l$-th and the $m-a+1$-th column of $C_{k, l, a, n \times m}^{i}$ is not less than $\left.n-k\right)$. Then Case 1 considered in Subsection 3.4 describes the rest rows of $C_{k, l, a, n \times m}^{i}$ and these rows correspond to the following function:

$$
\left.\mid \begin{array}{cccc}
k+3 & k+4 & \cdots & n \\
m-a-2 & m-a-3 & \cdots & m-a-(n-k-1)
\end{array}\right)
$$

note that $m-a-(n-k-1) \geqslant l$.

Assume that $m-a+1-l \leqslant n-k-1$ or equivalently $m-a-(n-k) \leqslant l-2$ (i.e., the distance between the $l$-th and the $m-a+1$-th column of $C_{k, l, a, n \times m}^{i}$ is less than $\left.n-k\right)$. Since each entry of the first column of $O^{r}\left(C_{k, l, a, n \times m}^{i}\right)$ equals 1, Case 2.1 considered in Subsection 3.4 (more precisely, the equality $l-1=k-a$ ) does not hold. So the inequality $k-a \leqslant l-2$ must hold. Then Case 2.2 considered in Subsection 3.4 describes the rest rows of $C_{k, l, a, n \times m}^{i}$ and these rows correspond to the following function:

$\left.\begin{array}{cccccccc}k+3 & k+4 & \ldots & k+m-a+1-l-1 & k+m-a+1-l & k+m-a+1-l+1 & \ldots & n \\ m-a-2 & m-a-3 & \ldots & l+1 & l & l-(k-a)-1 & \ldots & m-n+1\end{array}\right)$,

note that the inequality $m-a-(n-k) \leqslant l-2$ implies $k+m-a+1-l \leqslant n-1$.

Summarizing, we have proved the following result.

Proposition 82. Let $1 \leqslant k \leqslant n, 2 \leqslant l \leqslant m$ and $\max \{0, k-l+1\} \leqslant a \leqslant \min \{k, m+$ $1-l\}-1$. Then

(a) $C_{k, l, a, n \times m}^{i} \in I_{n}^{m}$.

(b) $f_{C_{k, l, a, n \times m}^{i}}$ is such that

(b.1) if $m-a-(n-k) \geqslant l-1$, then $f_{C_{k, l, a, n \times m}^{i}}=\left(\begin{array}{cccccc}1 & 2 & \ldots & a & a+1 \\ m & m-1 & \ldots & m-a+1 & l-1\end{array}\right.$

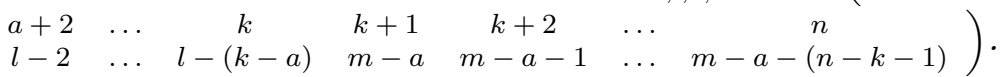

Note that $m-a-(n-k-1) \geqslant l$.

(b.2) if $m-a-(n-k) \leqslant l-2$, then $f_{C_{k, l, a, n \times m}^{i}}=\left(\begin{array}{ccccccc}1 & 2 & \cdots & a \\ m & m-1 & \cdots & m-a+1 & a+1 & \cdots-1\end{array} \quad \ldots\right.$

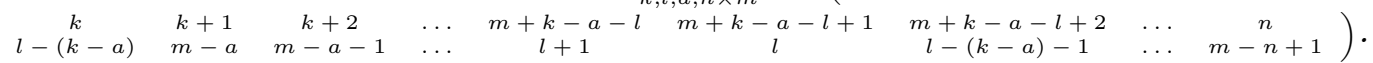
Note that $m-a-(n-k) \leqslant l-2$ implies $k-a \leqslant l-2$ (because $n \leqslant m)$, $l-(k-a)-1 \leqslant m-n+1$ and $m+k-a-l+1 \leqslant n-1$.

If $n=m$, then inequalities $m-a-(n-k) \geqslant l-1$ and $m-a-(n-k) \leqslant l-2$ are equivalent with $k-a=l-1$ (because $k-l+1 \leqslant \max \{0, k-l+1\} \leqslant a$ ) and $k-a \leqslant l-2$, respectively. Hence for $n=m$, cases (b.1) and (b.2) of Proposition 82 take the following forms:

(1) If $k-a=l-1$, then $f_{C_{k, l, a, n \times m}^{i}}=\left(\begin{array}{ccccccccc}1 & 2 & \ldots & a & a+1 & a+1 & \ldots & k & k+1 \\ n & n-1 & \ldots & n-a+1 & l-1 & l-2 & \ldots & 1 & n-a\end{array}\right.$

$$
\left.\begin{array}{ccc}
k+2 & \ldots & n \\
n-a-1 & \ldots & l
\end{array}\right) \text {. }
$$


(2) If $k-a \leqslant l-2$, then $f_{C_{k, l, a, n \times m}^{i}}=\left(\begin{array}{ccccccccc}1 & 2 & \ldots & a & a+1 & \ldots & k & k+1 \\ n & n-1 & \ldots & n-a+1 & l-1 & \ldots & l-(k-a) & n-a\end{array}\right.$ $\left.\begin{array}{ccccccc}k+2 & \ldots & n+k-a-l & n+k-a-l+1 & n+k-a-l+2 & \ldots & n \\ n-a-1 & \ldots & l+1 & l & l-(k-a)-1 & \ldots & 1\end{array}\right)$.

Of course, these two cases can be joined in one, because the equality $k-a=l-1$ implies that $l-(k-a)=1$ and $n+k-a+1-l=n$. Next, the inequality $k-a \leqslant l-1$ holds for all matrices $C_{k, l, a, n \times n}^{i}$. Thus we obtain the following result describing bijections corresponding to meet-irreducible elements of the lattice $\left(S G_{n}^{n}, \leqslant_{M}\right)$.

Corollary 83. Let $1 \leqslant k \leqslant n-1,2 \leqslant l \leqslant n$ and $\max \{0, k-l+1\} \leqslant a \leqslant \min \{k, n+$ $1-l\}-1$. Then

(a) $C_{k, l, a, n \times n}^{i} \in I_{n}^{n}$.

(b) $\begin{aligned} f_{C_{k, l, a, n \times n}^{i}} & =\left(\begin{array}{ccccccccccc}1 & 2 & \ldots & a & a+1 & \ldots & k & k+1 & k+2 & \ldots & n+k-a-l \\ n & n-1 & \ldots & n-a+1 & l-1 & \ldots & l-(k-a) & n-a & n-a-1 & \ldots & l+1 \\ n+k-a-l+1 & n+k-a-l+2 & \ldots & n \\ l & l-(k-a)-1 & \ldots & 1\end{array}\right) .\end{aligned}$

Applying Lemma 81 to Lemma 77, Theorem 78 and Proposition 79 we obtain the following three results for meet-irreducible elements of the lattice $\left(I G_{n}^{m}, \leqslant_{M}\right)$.

Lemma 84. Let $1 \leqslant k_{1}, k_{2} \leqslant n, 2 \leqslant l_{1}, l_{2} \leqslant m$ and $\max \left\{0, k_{1}-l_{1}+1\right\} \leqslant a_{1} \leqslant$ $\min \left\{k_{1}, m+1-l_{1}\right\}-1, \max \left\{0, k_{2}-l_{2}+1\right\} \leqslant a_{2} \leqslant \min \left\{k_{2}, m+1-l_{2}\right\}-1$. Then

$$
C_{k_{1}, l_{1}, a_{1}, n \times m}^{i} \leqslant{ }_{M} C_{k_{2}, l_{2}, a_{2}, n \times m}^{i} \quad \text { iff } \quad\left\{\begin{array}{l}
k_{1}-a_{1} \geqslant k_{2}-a_{2} \\
l_{1}+a_{1} \leqslant l_{2}+a_{2} \\
a_{1} \leqslant a_{2} \\
a_{1}+l_{1}-k_{1} \leqslant a_{2}+l_{2}-k_{2}
\end{array}\right.
$$

$$
C_{k_{1}, l_{1}, a_{1}, n \times m}^{i}=C_{k_{2}, l_{2}, a_{2}, n \times m}^{i} \quad \text { iff } \quad\left(k_{1}, l_{1}, a_{1}\right)=\left(k_{2}, l_{2}, a_{2}\right) .
$$

Proof. (a): By Corollary 22 and Lemma 81 we have that $C_{k_{1}, l_{1}, a_{1}, n \times m}^{i} \leqslant{ }_{M} C_{k_{2}, l_{2}, a_{2}, n \times m}^{i}$ iff $\left(C_{k_{1}, l_{1}, a_{1}, n \times m}^{i}\right)^{t d} \leqslant M \quad\left(C_{k_{2}, l_{2}, a_{2}, n \times m}^{i}\right)^{t d}$ iff $B_{k_{2}, m-l_{2}+2, k_{2}-a_{2}, n \times m}^{i} \leqslant M \quad B_{k_{1}, m-l_{1}+2, k_{1}-a_{1}, n \times m}^{i}$. Next, by Lemma 77(a) we obtain

$$
B_{k_{2}, m-l_{2}+2, k_{2}-a_{2}, n \times m}^{i} \leqslant{ }_{M} B_{k_{1}, m-l_{1}+2, k_{1}-a_{1}, n \times m}^{i}
$$

iff

$$
\left\{\begin{array}{l}
k_{2}-\left(k_{2}-a_{2}\right) \geqslant k_{1}-\left(k_{1}-a_{1}\right) \\
\left(m-l_{2}+2\right)+\left(k_{2}-a_{2}\right) \leqslant\left(m-l_{1}+2\right)+\left(k_{1}-a_{1}\right) \\
k_{2}-a_{2} \leqslant k_{1}-a_{1} \\
\left(k_{2}-a_{2}\right)+\left(m-l_{2}+2\right)-k_{2} \leqslant\left(k_{1}-a_{1}\right)+\left(m-l_{1}+2\right)-k_{1}
\end{array}\right.
$$

iff

$$
\left\{\begin{array}{l}
a_{1} \leqslant a_{2} \\
a_{1}+l_{1}-k_{1} \leqslant a_{2}+l_{2}-k_{2} \\
k_{1}-a_{1} \geqslant k_{2}-a_{2} \\
l_{1}+a_{1} \leqslant l_{2}+a_{2}
\end{array} .\right.
$$

THE ELECtronic Journal of COMBINATORics 23(1) (2016), \#P1.3 
The point (b) follows from (a). Of course, it is also implied by Lemmas 77(b) and 81 . Note that this fact may be obtained by using the form of matrices $O^{r}\left(C_{k, l, a, n \times m}^{p}\right)$, which is described above. But this is a very complicated method in this case.

Theorem 85. $\left(\operatorname{Mir}\left(I G_{n}^{m}\right), \leqslant_{M}\right)$ is isomorphic to the poset $\left(R_{n, m}, \leqslant_{\text {prod }}\right)$ defined in Theorem 78, recall that $\left(R_{n, m}, \leqslant\right.$ prod $)$ is a subposet of the poset $\left(\mathbb{N}^{4}, \leqslant\right.$ prod $)$ such that $R_{n, m}=$ $\left\{(x, y, z, t) \in \mathbb{N}^{4}: 1 \leqslant x \leqslant n, 2 \leqslant y \leqslant m, 1 \leqslant z \leqslant \min \{x, y-1\}, n+1 \leqslant x+y-z\right.$ and $t=$ $x+y-z\}$. Moreover, this isomorphism is given by the function

$$
C_{k, l, a, n \times m}^{i} \longmapsto(n-k+a+1, l+a, a+1, a+l-k+n) .
$$

Proof. Having Lemma 84(b) we can take a function $\Psi: \operatorname{Mir}\left(I G_{n}^{m}\right) \longrightarrow \mathbb{N}^{4}$ such that

$$
\Psi\left(C_{k, l, a, n \times m}^{i}\right)=(n-k+a+1, l+a, a+1, a+l-k)+(0,0,0, n) .
$$

Lemma 84(a) implies that $\Psi$ is an embedding of $\left(\operatorname{Mir}\left(I G_{n}^{m}\right), \leqslant_{M}\right)$ into $\left(\mathbb{N}^{4}, \leqslant_{\text {prod }}\right)$. Next, inequalities $\max \{0, k-l+1\} \leqslant a \leqslant \min \{k-1, m-l\}$ imply $1 \leqslant a+1 \leqslant n-k+a+1 \leqslant n$, $2 \leqslant 2+a \leqslant l+a \leqslant m$ and $a+l-k \geqslant 1$. Moreover, $(n-k+a+1)+(l+a)-(a+1)=$ $a+l-k+n \geqslant 1+n$. Hence it follows that $\Psi\left(\operatorname{Mir}\left(I G_{n}^{m}\right)\right) \subseteq R_{n, m}$. On the other hand, take $(x, y, z, t) \in R_{n, m}$. By the last part of the proof of Theorem 78 we have that

$$
1 \leqslant n-x+z \leqslant n, \quad 2 \leqslant y-z+1 \leqslant m
$$

and

$\max \{0,(n-x+z)-(y-z+1)+1\} \leqslant z-1 \leqslant \min \{n-x+z, m+1-(y-z+1)\}-1$.

Thus we can take the matrix $C_{n-x+z, y-z+1, z-1, n \times m}^{i}$. Of course, $\Psi\left(C_{n-x+z, y-z+1, z-1, n \times m}^{i}\right)=$ $(x, y, z, x+y-z)=(x, y, z, t)$. Hence $R_{n, m} \subseteq \Psi\left(\operatorname{Mir}\left(I G_{n}^{m}\right)\right)$, because $(x, y, z, t) \in R_{n, m}$ was arbitrarily chosen.

Proposition 86. $\left|\operatorname{Mir}\left(I G_{n}^{m}\right)\right|=\frac{3 m n(m-n)+(n-1) n(n+1)}{6}$.

In particular, $\left|\operatorname{Mir}\left(S G_{n}^{n}\right)\right|=\frac{(n-1) n(n+1)}{6}$.

Proof. It is obtained by Proposition 79, because $\left|\operatorname{Mir}\left(I G_{n}^{m}\right)\right|=\left|\left\{A^{d}: A \in \operatorname{Jir}\left(I G_{n}^{m}\right)\right\}\right|=$ $\left|\operatorname{Jir}\left(I G_{n}^{m}\right)\right|$ (see Corollary 22).

Lemma $84\left(\right.$ a) can be proved without using the self-duality of lattice $\left(I G_{n}^{m}, \leqslant_{M}\right)$ in a similar way as Lemma 77(a) (technically complicated details are left to the reader). Thus we can also obtain an alternative proof of Theorem 85, which do not use this self-duality (i.e., Corollary 22 and Lemma 81). Of course, Proposition 86 can be also shown without using this self-duality in the same way as Proposition 79.

By Theorems 78 and 85, functions $B_{k, l, a, n \times m}^{i} \longmapsto(n-k+a, l+a-1, a, a+l-k+n-1)$ and $C_{k, l, a-1, n \times m}^{i} \longmapsto(n-k+(a-1)+1, l+(a-1),(a-1)+1,(a-1)+l-k+n)=$ $(n-k+a, l+a-1, a, a+l-k+n-1)$, where $\max \{0, k-l+1\}+1 \leqslant a \leqslant \min \{k, m+1-$ $l\}$, are isomorphisms of posets $\left(\operatorname{Jir}\left(I G_{n}^{m}\right), \leqslant_{M}\right)$ and $\left(\operatorname{Mir}\left(I G_{n}^{m}\right), \leqslant_{M}\right)$ onto $\left(R_{n, m}, \leqslant_{\text {prod }}\right)$, respectively. Hence we obtain the following result. 
Corollary 87. Posets $\left(\operatorname{Jir}\left(I G_{n}^{m}\right), \leqslant_{M}\right)$ and $\left(\operatorname{Mir}\left(I G_{n}^{m}\right), \leqslant_{M}\right)$ are isomorphic and this isomorphism is given by the function $B_{k, l, a, n \times m}^{i} \longmapsto C_{k, l, a-1, n \times m}^{i}$ for all $1 \leqslant k \leqslant n$, $2 \leqslant l \leqslant m$ and $\max \{0, k-l+1\}+1 \leqslant a \leqslant \min \{k, m+1-l\}$.

Since $\left(\operatorname{Jir}\left(I G_{n}^{m}\right), \leqslant_{M}\right)$ is isomorphic to the dual of $\left(\operatorname{Mir}\left(I G_{n}^{m}\right), \leqslant_{M}\right)$ and vice versa (see Corollary 22), we obtain by the above corollary that the following fact holds.

\section{Corollary 88.}

(a) The function $B_{k, l, a, n \times m}^{i} \longmapsto B_{k, m-l+2, k-a+1, n \times m}^{i}$ is an involutive anti-automorphism of $\left(\operatorname{Jir}\left(I G_{n}^{m}\right), \leqslant_{M}\right)$. In particular, the poset $\left(\operatorname{Jir}\left(I G_{n}^{m}\right), \leqslant_{M}\right)$ is self-dual.

(b) The function $C_{k, l, a, n \times m}^{i} \longmapsto C_{k, m-l+2, k-a-1, n \times m}^{i}$ is an involutive anti-automorphism of the poset $\left(\operatorname{Mir}\left(I G_{n}^{m}\right), \leqslant_{M}\right)$. In particular, the poset $\left(\operatorname{Mir}\left(I G_{n}^{m}\right), \leqslant_{M}\right)$ is self-dual.

c) The function $(x, y, z, t) \longmapsto(n-z+1, m+n-x-y+z+1, n-x+1, m+n-y+1)$ is an involutive anti-automorphism of the poset $\left(R_{n, m}, \leqslant_{\text {prod }}\right)$. In particular, the poset $\left(R_{n, m}, \leqslant\right.$ prod $)$ is self-dual.

Proof. (a) and (b): Take the isomorphism $\Psi: B_{k, l, a, n \times m}^{i} \longmapsto C_{k, l, a-1, n \times m}^{i}$ between posets $\left(\operatorname{Jir}\left(I G_{n}^{m}\right), \leqslant_{M}\right)$ and $\left(\operatorname{Mir}\left(I G_{n}^{m}\right), \leqslant_{M}\right)$ defined in Corollary 87. Next, by Corollary 22 we have that the function $\Phi: C_{k, l, a, n \times m}^{i} \longmapsto\left(C_{k, l, a, n \times m}^{i}\right)^{t d}=B_{k, m-l+2, k-a, n \times m}^{i}$ is an isomorphism of $\left(\operatorname{Mir}\left(I G_{n}^{m}\right), \leqslant_{M}\right)$ onto the dual of $\left(\operatorname{Jir}\left(I G_{n}^{m}\right), \leqslant_{M}\right)$. Thus the composition $\Phi \circ \Psi$ is an anti-automorphism of $\left(\operatorname{Jir}\left(I G_{n}^{m}\right), \leqslant_{M}\right)$. Moreover, $\Phi\left(\Psi\left(B_{k, l, a, n \times m}^{i}\right)\right)=$ $B_{k, m-l+2, k-a+1, n \times m}^{i}$. It is easy to verify that $\Phi \circ \Psi$ is an involution.

The composition $\Phi^{-1} \circ \Psi^{-1}$ is an anti-automorphism of $\left(\operatorname{Mir}\left(I G_{n}^{m}\right), \leqslant_{M}\right)$. Since $\Phi^{-1}: B_{k, l, a, n \times m}^{i} \longmapsto\left(B_{k, l, a, n \times m}^{i}\right)^{t d}=C_{k, m-l+2, k-a, n \times m}^{i}$ and $\Psi^{-1}: C_{k, l, a, n \times m}^{i} \longmapsto B_{k, l, a+1, n \times m}^{i}$, we have $\Phi^{-1}\left(\Psi^{-1}\left(C_{k, l, a, n \times m}^{i}\right)\right)=C_{k, m-l+2, k-a-1, n \times m}^{i}$. It is easy to verify that $\Phi^{-1} \circ \Psi^{-1}$ is an involution.

(c): Let $\Theta: B_{k, l, a, n \times m}^{i} \longmapsto(n-k+a, l+a-1, a, a+l-k+n-1)$ be the isomorphism between posets $\left(\operatorname{Jir}\left(I G_{n}^{m}\right), \leqslant_{M}\right)$ and $\left(R_{n, m}, \leqslant_{\text {prod }}\right)$ defined in Theorem 78 . Then the composition $\Theta \circ(\Phi \circ \Psi) \circ \Theta^{-1}$ is an anti-automorphism of $\left(R_{n, m}, \leqslant_{\text {prod }}\right)$.

Next, take $(x, y, z, t) \in R_{n, m}$. It is easy to see $\Theta^{-1}((x, y, z, t))=B_{n-x+z, y-z+1, z, n \times m}^{i}$ (recall that $t=x+y-z$ ). Thus

$$
\begin{gathered}
\Theta\left((\Phi \circ \Psi)\left(\Theta^{-1}((x, y, z, t))\right)\right)=\Theta\left((\Phi \circ \Psi)\left(B_{n-x+z, y-z+1, z, n \times m}^{i}\right)\right)= \\
\Theta\left(B_{n-x+z, m-(y-z+1)+2,(n-x+z)-z+1, n \times m}^{i}\right)=\Theta\left(B_{n-x+z, m-y+z+1, n-x+1, n \times m}^{i}\right)= \\
(n-(n-x+z)+(n-x+1),(m-y+z+1)+(n-x+1)-1, \\
n-x+1,(n-x+1)+(m-y+z+1)-(n-x+z)+n-1)= \\
(n-z+1, m+n-x-y+z+1, n-x+1, m+n-y+1) .
\end{gathered}
$$

Using this formula it is not difficult to verify that $\Theta \circ(\Phi \circ \Psi) \circ \Theta^{-1}$ is also an involution. 
Since $\left(I G_{n}^{m}, \leqslant_{M}\right)$ is a finite distributive lattice, its dual $\left(I G_{n}^{m}, \leqslant_{M}^{d}\right)$ is isomorphic to the lattice of all order filters of $\left(\operatorname{Mir}\left(I G_{n}^{m}\right), \leqslant_{M}\right)$ (see [4] and Subsection 1.1). Hence and by Theorem 85 , since $\left(I G_{n}^{m}, \leqslant_{M}\right)$ is self-dual (see Corollary 22 ), we obtain

Corollary 89. $\left(I G_{n}^{m}, \leqslant_{M}\right) \simeq\left(O F\left(R_{n, m}\right), \subseteq\right)$.

By this corollary we obtain another proof of the fact that $\left|I G_{n}^{m}\right|$ is equal to the cardinality of the family of all anti-chains of $\left(R_{n, m}, \leqslant\right.$ prod $)$ (see Corollary 80 ).

Since Theorem 85 can be shown without using the self-duality of lattice $\left(I G_{n}^{m}, \leqslant_{M}\right)$, we can apply this result to obtain another proof of the self-duality of this lattice. More precisely, the lattice $\left(I G_{n}^{m}, \leqslant_{M}\right)$ is isomorphic to $\left(O I\left(R_{n, m}\right), \subseteq\right)$ (see Corollary 80), its dual $\left(I G_{n}^{m}, \leqslant_{M}^{d}\right)$ is isomorphic to $\left(O F\left(\operatorname{Mir}\left(I G_{n}^{m}\right), \subseteq\right)\right.$ (see [4] and Subsection 1.1) and $\left(O F\left(\operatorname{Mir}\left(I G_{n}^{m}\right), \subseteq\right) \simeq\left(O F\left(R_{n, m}\right), \subseteq\right)\right.$ (by Theorem 85). Next, the poset $\left(R_{n, m}, \leqslant_{\text {prod }}\right)$ is self-dual (see Corollary 88), so $\left(O I\left(R_{n, m}\right), \subseteq\right) \simeq\left(O I\left(R_{n, m}^{d}\right), \subseteq\right)$, where $R_{n, m}^{d}$ is the dual of $R_{n, m}$. Of course, $O I\left(R_{n, m}^{d}\right)=O F\left(R_{n, m}\right)$.

\subsection{Dedekind-MacNeille completion of posets $\left(I_{n}^{m}, \leqslant_{M}\right)$ and $\left(I(n, m), \leqslant_{F}\right)$}

Similarly as in two previous subsections we also assume here that $n \leqslant m$. Then by Corollary 37 and Propositions 75(a), 82(a) we obtain the following description of DedekindMacNeille completion of the poset $\left(I_{n}^{m}, \leqslant_{M}\right)$ (see also Proposition 10 and Corollary 22).

Theorem 90. $\left(L\left(I_{n}^{m}\right), \subseteq\right) \simeq\left(I G_{n}^{m}, \leqslant_{M}\right)$ (i.e., the Dedekind-MacNeille completion of the poset $\left(I_{n}^{m}, \leqslant_{M}\right)$ is isomorphic to $\left.\left(I G_{n}^{m}, \leqslant_{M}\right)\right)$, in particular, $\left(L\left(I_{n}^{m}\right), \subseteq\right)$ is a finite self-dual distributive lattice. Moreover, $\operatorname{Jir}\left(I G_{n}^{m}\right)=\operatorname{Jir}\left(I_{n}^{m}\right)$ and $\operatorname{Mir}\left(I G_{n}^{m}\right)=\operatorname{Mir}\left(I_{n}^{m}\right)$.

We know that the poset $\left(I(n, m), \leqslant_{F}\right)$, thus also $\left(I_{n}^{m}, \leqslant_{M}\right)$, is self-dual (see Corollary 3), so their Dedekind-MacNeille completions are also self-dual (see Proposition 33). Hence we obtain another proof of the fact that the lattice $\left(I G_{n}^{m}, \leqslant_{M}\right)$ is self-dual (see Corollary 22). But this proof does not give a formula for any involutive anti-isomorphism of $\left(I G_{n}^{m}, \leqslant_{M}\right)$.

Since the function $f \longmapsto M(f)$ is an isomorphism between posets $\left(I(n, m), \leqslant_{F}\right)$ and $\left(I_{n}^{m}, \leqslant_{M}\right)$, the following properties of $\left(I(n, m), \leqslant_{F}\right)$ are obtained by Theorem 90 and Propositions 75(b), 82(b).

\section{Corollary 91.}

(a) $(L(I(n, m)), \subseteq) \simeq\left(I G_{n}^{m}, \leqslant_{M}\right)$, i.e., the Dedekind-MacNeille completion of the poset $\left(I(n, m), \leqslant_{F}\right)$ is isomorphic to $\left(I G_{n}^{m}, \leqslant_{M}\right)$. In particular, $(L(I(n, m)), \subseteq)$ is a finite self-dual distributive lattice.

(b) $\operatorname{Jir}(I(n, m))=\left\{f_{B_{k, l, a, n \times m}^{i}}: 1 \leqslant k \leqslant n, 2 \leqslant l \leqslant m, \max \{0, k-l+1\}+1 \leqslant\right.$ $a \leqslant \min \{k, m+1-l\}\}=\left\{\begin{array}{ccccccccc}1 & 2 & \ldots & k-a & k-a+1 & k-a+2 & \ldots & k & k+1 \\ 1 & 2 & \ldots & k-a & l & l+1 & \ldots & l+a-1 & k-a+1\end{array}\right.$

$\left.\begin{array}{ccccccc}k+2 & \ldots & l+a-1 & l+a & l+a+1 & \ldots & n \\ k-a+2 & \ldots & l-1 & l+a & l+a+1 & \ldots & n\end{array}\right): 1 \leqslant k \leqslant n, 2 \leqslant l \leqslant m, \max \{0, k-$ $l+1\}+1 \leqslant a \leqslant \min \{k, m+1-l\}$ and $l+a-1 \leqslant n\} \cup\left\{\begin{array}{ccccc}1 & 2 & \ldots & k-a & k-a+1 \\ 1 & 2 & \ldots & k-a & l\end{array}\right.$ 
$\left.\begin{array}{ccccccc}k-a+2 & \ldots & k & k+1 & k+2 & \ldots & n \\ l+1 & \ldots & l+a-1 & k-a+1 & k-a+2 & \ldots & (k-a)+(n-k)=n-a\end{array}\right): 1 \leqslant k \leqslant n, 2 \leqslant$ $l \leqslant m, \max \{0, k-l+1\}+1 \leqslant a \leqslant \min \{k, m+1-l\}$ and $l+a-1 \geqslant n+1\}$.

(c) $\operatorname{Mir}(I(n, m))=\left\{f_{C_{k, l, a, n \times m}^{i}}: 1 \leqslant k \leqslant n, 2 \leqslant l \leqslant m, \max \{0, k-l+1\} \leqslant\right.$ $a \leqslant \min \{k, m+1-l\}-1\}=\left\{\begin{array}{ccccccccc}1 & 2 & \ldots & a & a+1 & a+2 & \ldots & k \\ m & m-1 & \ldots & m-a+1 & l-1 & l-2 & \ldots & l-(k-a)\end{array}\right.$ $\left.\begin{array}{cccc}k+1 & k+2 & \ldots & n \\ m-a & m-a-1 & \ldots & m-a-(n-k-1)\end{array}\right): 1 \leqslant k \leqslant n, 2 \leqslant l \leqslant m, \max \{0, k-l+1\} \leqslant$ $a \leqslant \min \{k, m+1-l\}-1$ and $m-a-(n-k) \geqslant l-1\} \cup\left\{\left(\begin{array}{cccc}1 & 2 & \ldots & a \\ m & m-1 & \ldots & m-a+1\end{array}\right.\right.$ $\begin{array}{llllllllll}a+1 & \ldots & k & k+1 & k+2 & \ldots & m+k-a-l & m+k-a-l+1 & m+k-a-l+2 & \ldots\end{array}$

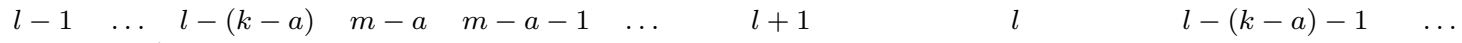
$\left.\begin{array}{c}n \\ m-n+1\end{array}\right): 1 \leqslant k \leqslant n, 2 \leqslant l \leqslant m, \max \{0, k-l+1\} \leqslant a \leqslant \min \{k, m+1-$ $l\}-1$ and $m-a-(n-k) \leqslant l-2\}$.

By Corollary 91, Theorems 78, 85 and Corollaries 87, 88 we obtain the following result which describes the structure of subposets of $\left(I(n, m), \leqslant_{F}\right)$ induced by join- and meet-irreducible elements.

Corollary 92. Let $R_{n, m}=\{(x, y, z, t): 1 \leqslant x \leqslant n, 2 \leqslant y \leqslant m, 1 \leqslant z \leqslant \min \{x, y-$ $1\}, n+1 \leqslant x+y-z$ and $t=x+y-z\}$. Then

(a) $\left(\operatorname{Jir}(I(n, m)), \leqslant_{F}\right)$ and $\left(\operatorname{Mir}(I(n, m)), \leqslant_{F}\right)$ are isomorphic to $\left(R_{n, m}, \leqslant_{\text {prod }}\right)$. Moreover, these isomorphisms are given by functions

$$
f_{B_{k, l, a, n \times m}^{i}} \longmapsto(n-k+a, l+a-1, a, a+l-k+n-1)
$$

and

$$
f_{C_{k, l, a, n \times m}^{i}} \longmapsto(n-k+a+1, l+a, a+1, a+l-k+n) .
$$

(b) The function $f_{B_{k, l, a, n \times m}^{i}} \longmapsto f_{C_{k, l, a-1, n \times m}^{i}}$ is an isomorphism of $\left(\operatorname{Jir}(I(n, m)), \leqslant_{F}\right)$ onto $\left(\operatorname{Mir}(I(n, m)), \leqslant_{F}\right)$.

(c) Functions $f_{B_{k, l, a, n \times m}^{i}} \longmapsto f_{B_{k, m-l+2, k-a+1, n \times m}^{i}}$ and $f_{C_{k, l, a, n \times m}^{i}} \longmapsto f_{C_{k, m-l+2, k-a-1, n \times m}^{i}}$ are involutive anti-automorphisms of $\left(\operatorname{Jir}(I(n, m)), \leqslant_{F}\right)$ and $\left(\operatorname{Mir}(I(n, m)), \leqslant_{F}\right)$, respectively. In particular, $\left(\operatorname{Jir}(I(n, m)), \leqslant_{F}\right)$ and $\left(\operatorname{Mir}(I(n, m)), \leqslant_{F}\right)$ are self-dual posets.

Of course, self-duality of posets $\left(\operatorname{Jir}(I(n, m)), \leqslant_{F}\right)$ and $\left(\operatorname{Mir}(I(n, m)), \leqslant_{F}\right)$ follows also from (b) and Corollary 3. But by Corollary 88 we have formulas for their antiautomorphisms.

Finally, note that taking $n=m$ we obtain particular cases of all the above results for posets $\left(S_{n}^{n}, \leqslant_{M}\right)$ and $\left(S(n), \leqslant_{B}\right)$. For example, $\left(S G_{n}^{n}, \leqslant_{M}\right)$ is (up to isomorphism) Dedekind-MacNeille completion of these two posets. In particular, $\left(L\left(S_{n}^{n}\right), \subseteq\right)$ and $(L(S(n)), \subseteq)$ are finite self-dual distributive lattices. Next,

$$
\operatorname{Jir}\left(S_{n}^{n}\right)=\operatorname{Jir}\left(S G_{n}^{n}\right), \quad \operatorname{Mir}\left(S_{n}^{n}\right)=\operatorname{Mir}\left(S G_{n}^{n}\right)
$$


and

$$
\begin{aligned}
& \operatorname{Jir}(S(n))=\left\{f_{A}: A \in \operatorname{Jir}\left(S_{n}^{n}\right)\right\}=\left\{f_{B_{k, l, a, n \times n}^{i}}: 1 \leqslant k \leqslant n-1,2 \leqslant l \leqslant n, \max \{0, k-l+\right. \\
& 1\}+1 \leqslant a \leqslant \min \{k, n+1-l\}\}=\left\{\begin{array}{ccccccccc}
1 & 2 & \ldots & k-a & k-a+1 & k-a+2 & \ldots & k & k+1 \\
1 & 2 & \ldots & k-a & l & l+1 & \ldots & l+a-1 & k-a+1
\end{array}\right. \\
& \left.\begin{array}{ccccccc}
k+2 & \ldots & l+a-1 & l+a & l+a+1 & \ldots & n \\
k-a+2 & \ldots & l-1 & l+a & l+a+1 & \ldots & n
\end{array}\right): 1 \leqslant k \leqslant n-1,2 \leqslant l \leqslant n, \max \{0, k-l+ \\
& 1\}+1 \leqslant a \leqslant \min \{k, n+1-l\}\} \text {, }
\end{aligned}
$$
$n, \max \{0, k-l+1\} \leqslant a \leqslant \min \{k, n+1-l\}-1\}$.

Moreover, posets $\left(\operatorname{Jir}(S(n)), \leqslant_{B}\right)$ and $\left(\operatorname{Mir}(S(n)), \leqslant_{B}\right)$ are self-dual and isomorphic to $\left(R_{n, n}, \leqslant_{\text {prod }}\right)$, in particular, $\left(\operatorname{Jir}(S(n)), \leqslant_{B}\right) \simeq\left(\operatorname{Mir}(S(n)), \leqslant_{B}\right)$.

\section{References}

[1] A. Bjorner and F. Brenti. Combinatorics of Coxeter Groups. Graduate Text in Mathematics 231, Springer, 2005.

[2] D. M. Bressoud. Proofs and Confirmations. The Story of the Alternating Sign Matrix Conjecture. Spectrum series. The Mathematical Association of America, 1999.

[3] S. Burris and H. P. Sankappanavar. A Course in Universal Algebra. Graduate Texts in Mathematics, 78. Springer-Verlag, New York-Berlin, 1981.

[4] P. Crawley and R. P. Dilworth. Algebraic Theory of Lattices. Prentice Hall Inc., Englewood Cliffs, NJ, 1973.

[5] B. A. Davey and H. A. Priestley. Introduction to Lattices and Order, Cambridge University Press 2nd ed. 2002.

[6] M. Fortin. Treillis Enveloppant des Fonctions Partielles Injectives. PhD thesis, Université du Québec à Montréal, 2007.

[7] M. Fortin. The MacNeille Completion of the Poset of Partial Injective Functions. Electron. J. Combin., 15, R62, 2008.

[8] M. Geck and S. Kim. Bases for the Bruhat-Chevalley Order on All Finite Coxeter Groups. J. Algebra, 197(1): 278-310, 1997.

[9] G. Grätzer. General Lattice Theory. Birkhäuser, 2nd ed., 2003.

[10] A. Lascoux and M. P. Schützenberger. Treillis et bases des groupes de Coxeter. Electron. J. Combin., 3, R27, 1996.

[11] N. Reading. Order Dimension, Strong Bruhat Order and Lattice Properties for Posets. Order, 19: 73-100, 2002.

[12] L. E. Renner. Linear Algebraic Monoids. Encyclopaedia of Mathematical Sciences, vol. 134. Springer, 2005.

[13] R. P. Stanley. Enumerative Combinatorics. Cambridge University Press, $2^{\text {nd }}$ ed., 2012. 